\title{
Hybrid Strength Two Covering Array Constructions: Using Cover Starters to Create Covering Arrays
}

by

Jason R. Lobb

\author{
A thesis submitted to \\ the Faculty of Graduate Studies and Research \\ in partial fulfilment of the requirements for the degree of
}

\section{Master of Science}

School of Mathematics and Statistics

Ottawa-Carleton Institute for Mathematics and Statistics

Carleton University

Ottawa, Ontario, Canada

January 2010

(C) Copyright 2010

Jason R. Lobb 


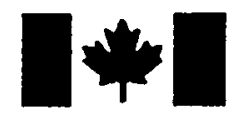

\section{Library and Archives}

Canada

Published Heritage

Branch

395 Wellington Street Ottawa ON K1A ON4 Canada
Bibliothèque et

Archives Canada

Direction du

Patrimoine de l'édition

395 , rue Wellington

Ottawa ON K1A ON4

Canada
Your file Votre référence

ISBN: 978-0-494-64434-8

Our file Notre référence

ISBN: 978-0-494-64434-8
NOTICE:

The author has granted a nonexclusive license allowing Library and Archives Canada to reproduce, publish, archive, preserve, conserve, communicate to the public by telecommunication or on the Internet, loan, distribute and sell theses worldwide, for commercial or noncommercial purposes, in microform, paper, electronic and/or any other formats.

The author retains copyright ownership and moral rights in this thesis. Neither the thesis nor substantial extracts from it may be printed or otherwise reproduced without the author's permission.
AVIS:

L'auteur a accordé une licence non exclusive permettant à la Bibliothèque et Archives Canada de reproduire, publier, archiver, sauvegarder, conserver, transmettre au public par télécommunication ou par l'Internet, prêter, distribuer et vendre des thèses partout dans le monde, à des fins commerciales ou autres, sur support microforme, papier, électronique et/ou autres formats.

L'auteur conserve la propriété du droit d'auteur et des droits moraux qui protège cette thèse. $\mathrm{Ni}$ la thèse ni des extraits substantiels de celle-ci ne doivent être imprimés ou autrement reproduits sans son autorisation.
In compliance with the Canadian Privacy Act some supporting forms may have been removed from this thesis.

While these forms may be included in the document page count, their removal does not represent any loss of content from the thesis.
Conformément à la loi canadienne sur la protection de la vie privée, quelques formulaires secondaires ont été enlevés de cette thèse.

Bien que ces formulaires aient inclus dans la pagination, il n'y aura aucun contenu manquant.

\section{Canadä}


For my Mom

[ii] 


\section{Abstract}

A covering array $C A_{\lambda}(N ; t, k, v)$ is a $k \times N$ array on a set of $v$ symbols with the property that in each of its $t \times N$ subarrays every $t$-tuple with entries from $v$ is in at least $\lambda$ columns. This thesis examines eight constructions for covering arrays that employ a cover starter $\kappa \times 1$ array. Two of these constructions have been previously researched. This thesis uses previously unused permutation groups, expanded ranges of search, and six new constructions to deepen the knowledge of cover starter based constructions. New least upper bounds on covering arrays are produced, new lower bounds on the spectra of existences of the cover starters and patterns of existence of orthogonal arrays by these constructions are proven. 
First thank to my supervisors Dr. Brett Stevens and Dr. Peter Danziger for their assistance and guidance. They had an ocean of patience. Thanks to Brett for his ever positive attitude and Peter for his steadying hand. Thanks also to Valerie Daley for all her assistance and care.

Without my parents emotional and financial support I would not have been able to finish this degree. My mom will always be my coach and my biggest regret is not finishing this in time for her to see. My dad was a rock and without him I would be lost. Thanks as well to Heather, Boris, Olivia and Anneka.

The friendship and support of Petar and Magda Duspara and Phil McGreevy was invaluable. Many others helped along the way: Lara, Lissa, Seth, Mel, Terra, Sam, Adriana, Brent, Jay, Megan are some but there are many more. Thanks to the Batey-ites for allowing me to be a surrogate lab member and to the staff of Mike's, Molly's (especially Beth), and Mitzi's. Thanks to the members of Grand Trunk and Carleton Ultimate. 


\section{Contents}

$\begin{array}{ll}\text { Abstract } & \text { iii }\end{array}$

Acknowledgements iv

Contents v

1 Introduction 1

2 Related Designs and Permutation Groups 6

2.1 Introduction . . . . . . . . . . . . . . . . 6

2.2 Covering Arrays and Orthogonal Arrays $\ldots \ldots \ldots$

2.3 Mutually Orthogonal Latin Squares . . . . . . . . . . . . . 9

2.4 Some Constructions for Orthogonal and Covering Arrays . . . 13

2.5 Permutation Groups and Difference Designs . . . . . . . 16

3 Background and Techniques of the Constructions 21

3.1 Introduction . . . . . . . . . . . . . . . . . . . . 21

3.2 Covering Orbit Representatives in Subarrays: The $\left[M^{G}, C\right]$

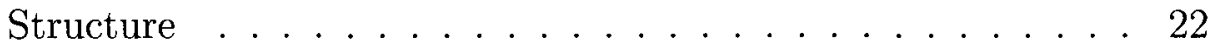

3.3 Cover Starter Generation of a Subarray . . . . . . . . . . 25 
3.4 Analysis of Past Results . . . . . . . . . . . . . . 31

4 The Constructions and Search Methods 35

4.1 Introduction . . . . . . . . . . . . . . 35

4.2 No Fixed Points . . . . . . . . . . . . . . . . . 37

4.2.1 Introduction and the Search Reductions . . . . . . 37

$4.2 .2 \quad$ Entire- $k$ Style Construction . . . . . . . . . . . . 39

4.2 .3 Partial- $k$ Style Construction . . . . . . . . . . . . 40

4.3 Single Fixed Point . . . . . . . . . . . . . . . . . 43

4.3.1 Introduction and the Search Reductions . . . . . . 43

4.3.2 Entire- $k$ Style Construction . . . . . . . . . . . . 45

4.3 .3 Partial- $k$ Style Construction . . . . . . . . . . . . 46

4.4 Double Fixed Point . . . . . . . . . . . . . . . . . . . . 49

4.4.1 Introduction and the Simple Construction . . . . . . 49

4.4.2 The Augmented Constructions . . . . . . . . . . . . . . 51

4.4 .3 The Search Reductions . . . . . . . . . . . . . . . 58

4.4.4 Entire- $k$ Style Constructions . . . . . . . . . . . . . 60

4.4.5 Partial- $k$ Style Constructions . . . . . . . . . . 63

4.5 Description of the searches . . . . . . . . . . . 68

5 Results and Analysis $\quad 75$

5.1 Results of Searches and New Least Upper Bounds . . . . . . 76

5.2 Comparison of Size and Lower Bounds Found for Covering Arrays Produced by Each Construction . . . . . . . . . . . 82

5.3 Gaps in the Spectra of $k$ values for a Given Group and Construction . . . . . . . . . . . . . . 89 
5.3 .1 Introduction . . . . . . . . . . . . 89

5.3 .2 Self Paired Orbitals . . . . . . . . . . . . . . 90 90

5.3.3 Analysis of Existences That Provide Orthogonal Arrays 94

5.4 Expected Existence of Starters . . . . . . . . . . . . . . 99

6 Conclusions and Future Work 101

6.1 Contributions . . . . . . . . . . . . . . . . 104

6.2 Future Work . . . . . . . . . . . . . . 105

$\begin{array}{ll}\text { A Cover Starter Examples } & 108\end{array}$ 


\section{Chapter 1}

\section{Introduction}

Development and testing of component-wise systems involves complex interactions. Component-wise systems are prevalent in software development $[5,7,17,29]$. There are other fields where component-wise systems arise, such as hardware testing [13] and gene signalling pathways [22]. Checking every possible interaction between all the components for errors is infeasible in many cases. For instance if there are 8 components, also referred to as factors, and 11 settings for each component then the number of possible arrangements of this system is $8^{11}=8589934592$. Thus there is a desire to test for errors via cost- and time-efficient means.

The goal of much combinatorial testing research is to create test suites that find a large percentage of errors in a system and are efficient in the number of tests required. Covering arrays prove useful in locating a large percentage of errors in component-wise systems [5, 7, 17, 29].

Definition 1.1 (Covering Array). A $C A_{\lambda}(N ; t, k, v)$ is a $k \times N$ array with entries from a set $X$, which has $|X|=v$. The entries are arranged so that 
for any $t \times \lambda \cdot v^{t}$ subarray the columns contain every $t$-tuple of the set $X$ at least $\lambda$ times.

Covering arrays are combinatorial designs. Combinatorial designs are collections of subsets that have guaranteed coverage properties. More information on this field can be found in Beth, Jungnickel and Lenz [2], Colbourn and Dinitz [25], and Stinson [25].

A covering array of strength $t$ provides a test suite that can account for every $t$-wise interaction of a system. These require only a very small number of tests compared to the total number of possible arrangements. In the previous example, a covering array can test pair-wise interaction with 8 factors each having 11 settings with only 78 arrangements [21]. This is far more efficient compared to the 8589934592 arrangements required by exhaustive testing.

Covering arrays are found by two major methods: algebraic and by algorithmic search. Colbourn has written a thorough overview of methods [8] and many others have written on specific techniques $[4,10,22,24]$. This thesis presents eight hybrid constructions that combine an algebraic structure and algorithmic searches to build strength 2 covering arrays. Note that a similar hybrid approach has been used for $t>2[23,27]$.

The algebraic structure of the constructions use two arrays $M$ and $C$ and a permutation group $G$. The array $M$ is acted on by a permutation group, $G$, creating $|G|$ arrays the same size as $M$. These $|G|$ arrays are concatenated together horizontally to form $M^{G}$. Six of the constructions have an additional subarray, $C$, concatenated to $M^{G}$. This forms an array, $\left[M^{G}, C\right]$, which, due to conditions imposed on $M$ and $C$ with respect to $G$, is a covering array. 
These construction are referred to as hybrid since, in addition to the algebraic structure of the covering arrays formed, a $1 \times \kappa$ array, a cover starter, is searched for and determines the array $M$.

Two of these constructions have been previously researched. One by Meagher and Stevens $[19,20,21]$ and the other by Colbourn et al. [10]. These projects provide limited insight into the behaviour of cover starters, sparse existence data of the one construction, and only utilize cyclic groups $[10,19,20,21]$. In this thesis more expansive searching is performed in order to better understand where to expect cover starters to exist and all groups of a given order are investigated in order to find new least upper bounds on the parameter $N$. The lower the $N$ for a given $v, k, t$, and $\lambda$ the more efficient the covering array construction is. As well as expanding the existence data and using non-cyclic groups, six new variant constructions are introduced. For a given parameter set, two of the constructions are such that they are more efficient and the other four are less efficient yet exist for lower values of $k$ and could find least upper bounds in ranges where the other constructions can not exist.

The eight constructions studied have four types: the no fixed point, the single fixed point, the simple double fixed point, and the augmented double fixed point. There are two different styles of each of the types: the Entire-k and the Partial- $k$. Searches are used to find a cover starter, which is a $\kappa \times 1$ array.

Chapter 2 gives explicit definitions and the basic properties of covering arrays and orthogonal arrays, which are a special case of covering arrays. A brief discussion of related designs is provided and constructions that can be 
used to create covering arrays are given. The necessary theory of permutation groups is given.

The motivating construction with its $\left[M^{G}, C\right]$ format $[6]$ is described in Chapter 3. How to develop a circulant array from a cover starter is defined. The two constructions of Meagher and Stevens [19, 20,21] and Colbourn et al. [10] are surveyed. The prior exhaustive searching and least upper bounds achieved on covering arrays are surveyed for the two previously used constructions. The scope of how this thesis expands upon the previous work and some of the topics of analysis are given.

The fourth chapter outlines the eight constructions in detail and describes the search algorithms used. The searches are either an exhaustive search via a Gray code [15] or a heuristic hill climb [16]. The reductions of the exhaustive search space for the constructions are explained. Each construction's particular $M$, its naive bound on the number of factors, $k$, and proof that the constructions yields a covering array is given.

The results and analysis are given in Chapter 5. The new least upper bounds found by this thesis are stated. In this chapter we give the spectra of $k$ values for which cover starters exist by their group for each construction. An efficient procedure for producing new least upper bounds by these constructions in the future for higher values of $v$ is outlined from observations of which constructions are expected to yield least upper bounds for different values of $k$. The gaps in the existence spectra are explained by self paired orbitals and orthogonal arrays. The existence of self paired orbitals yields a lemma providing a new lower bound on the existence of cover starters. The patterns of the existences of orthogonal arrays built from cover starters ac- 
count for other gaps. Proofs of three cases of when orthogonal arrays exist are also given. The possibility of constructing a cover starter of length $k$ given a cover starter from smaller $k$ is discussed.

The conclusions and future work summarizes each chapter and highlight the contributions of this thesis. Future work includes using the constructions for higher parameter sets, proving of the conjecture of the one case of orthogonal array existence, proving of the conjecture of guaranteed existence of a cover starter of length $k$ when there exists on of $k-2$, and discussing the possibility of using cover starter like objects to create other designs.

The explicit cover starters found for each construction are listed in Appendix A. 


\section{Chapter 2}

\section{Related Designs and Permutation Groups}

\section{$2.1 \quad$ Introduction}

This chapter establishes the basic definitions and theorems that provide the base for work in this thesis. Many texts provide in depth information on these topics. The definitions and theorem statements for designs are described in Beth, Jungnickel and Lenz [1, 2], and Colbourn and Dinitz [9]. Many of the proofs in Sections 2.3 and 2.4 can be found in Stinson [25]. For the theory of permutation groups given in Section 2.5, Dixon [11] and Wielandt [28] were used as references. More information on groups and finite fields is in Dummit and Foote [12]. 


\subsection{Covering Arrays and Orthogonal Arrays}

Covering arrays, $C A_{\lambda}(N ; t, k, v)$, are a relaxation of the definition of orthogonal arrays, $O A_{\lambda}(t, k, v)$. These designs have $k$ of factors, which are represented as rows and $N$ denotes the number of columns. In some literature the transpose is used $[5,8,10,23,22,27,29,23,27]$. Each factor has its entries from a set $X$ where $|X|=v$.

Definition 2.1 (Orthogonal Array). An $O A_{\lambda}(t, k, v)$ is a $k \times \lambda v^{t}$ array with entries from a set $X$, which has $|X|=v$. The entries are arranged such that, for any $t \times \lambda v^{t}$ subarray, every $t$-tuple with entries from the set $X$ is contained in $\lambda$ columns.

The condition requiring an orthogonal array to have the $t$-tuples occurring exactly $\lambda$ times is restrictive. Many upper bounds have been found on $k$ [9]. For $\lambda=1$ and $t=2$ orthogonal arrays have a general upper bound $k \leq v+1$ by Theorem 2.3 and 2.4. Designs that ensure coverage conditions, yet are more relaxed in definition, are sought for parameter values for which orthogonal arrays do not exist. Covering arrays are natural extensions of orthogonal arrays. They require each $t$-tuple to be contained at least $\lambda$ times in each $t \times N$ subarray. Packing arrays are a complimentary design that have each $t$-tuple occur at most $\lambda$ times in each $t \times N$ subarray [9].

There are also objects called mixed covering arrays, $M C A_{\lambda}\left(N ; t, k,\left(v_{1}, \ldots, v_{k}\right)\right)$ [10] where the entries of each factor come from its own set which can vary in size. The size of the sets for each factor $1, \ldots, k$ are denoted respectively with $v_{1}, \ldots, v_{k}$ and $\left(v_{1}, \ldots, v_{k}\right)$ is called the profile of the $M C A$. In every $t \times N$ subarray that has the rows $r_{1}, \ldots, r_{t}$ 
there are $\lambda$ columns that contain $\left(x_{1}, \ldots, x_{t}\right)$ for every choice of $x_{i} \in X_{r_{i}}$. This type of array allows a more accurate modelling of problems but does not as easily allow algebraic methods to create them [10]. Finally note that a $C A_{\lambda}(N ; t, k, v)$ can be transformed into $M C A_{\lambda}\left(N ; t, k,\left(v_{1}, \ldots, v_{k}\right)\right)$ by reassigning the $C A_{\lambda}(N ; t, k, v)$ factors' set values when $v_{i} \leq v$ for all $i$.

Covering arrays can have any $N$ provided the covering condition on the $t$-tuples is met. A $C A_{\lambda}(N ; t, k, v)$ can always be created by listing all the $k$-tuples with entries from $X \lambda$ times. This creates a primitive upper bound on a covering array of $N \leq \lambda \cdot|X|^{k}$. If for values $\lambda_{0}, t_{0}, v_{0}, k_{0}, N_{0}$ a $C A_{\lambda_{0}}\left(N_{0} ; t_{0}, k_{0}, v_{0}\right)$ is known to have minimum $N$ then it is called an optimal covering array and $C A N_{\lambda_{0}}\left(t_{0}, k_{0}, v_{0}\right)=N$. The $C A N_{\lambda}(t, k, v)=N_{0}$ is referred to as the covering array number. Covering array numbers are difficult to find and thus many techniques to produce least upper bounds are sought $[4,6,8,10,22,19,20,21]$. If there exists an orthogonal array, it is an optimal covering array, but an optimal covering array need not be an orthogonal array. Also note that if there exists a $C A_{\lambda_{1}}\left(N ; t_{1}, k_{1}, v_{1}\right)$ then there exists a $C A_{\lambda_{2}}\left(N ; t_{2}, k_{2}, v_{2}\right)$ any $\lambda_{2} \leq \lambda_{1}, t_{2} \leq t_{1}, k_{2} \leq k_{1}$ and $v_{2} \leq v_{1}$.

In this thesis all covering arrays from the eight constructions have $\lambda=1$ and $t=2$ and from now on $O A(k, v)$ and $C A(N ; k, v)$ are assumed to be a $O A_{1}(2, k, v)$ and $C A_{1}(N ; 2, k, v)$ respectively. There are arrays with $t=3$ and $\lambda=1$ discussed in this thesis in Section 3.1 and those arrays are specifically noted as $O A(3, k, v)$ and $C A(N ; 3, k, v)$ as appropriate. 


\subsection{Mutually Orthogonal Latin Squares}

There are many combinatorial objects that are equivalent to orthogonal arrays. Transversal designs, affine planes, and sets of mutually orthogonal Latin squares are examples of such designs. Orthogonal Latin squares are the oldest of these designs as they have their roots in the thirty six officer problem proposed by Euler in 1781 [3, 9]. The study of mutually orthogonal Latin squares, MOLS, has provided a great source of orthogonal arrays. The equivalence between a set of MOLS and an $O A$ is shown in Theorem 2.4.

A Latin square of size $n$ is an $n \times n$ array where each cell has an entry from the symbol set $X=\{1,2, \ldots, n\}$ so that each element occurs once in each row and column. For two Latin squares, $S_{1}$ and $S_{2}$, to be orthogonal they must have the property that there are no two pairs $\left(s_{1_{i j}}, s_{2_{i j}}\right)$ and $\left(s_{1_{l m}}, s_{2_{l m}}\right)$ such that $\left(s_{1_{i j}}, s_{2_{i j}}\right)=\left(s_{1_{l m}}, s_{2_{l m}}\right)$ unless $i=l$ and $j=m$. Since there are $n^{2}$ cells, all possible $n^{2}$ pairs from $X \times X$ are present. Table 2.1 gives an example of three mutually orthogonal Latin squares where $n=4$.

Table 2.1: Three mutually orthogonal Latin squares of order four.

$\begin{array}{llllllllllll}1 & 2 & 3 & 4 & 1 & 2 & 3 & 4 & 1 & 2 & 3 & 4 \\ 4 & 3 & 2 & 1 & 3 & 4 & 1 & 2 & 2 & 1 & 4 & 3 \\ 2 & 1 & 4 & 3 & 4 & 3 & 2 & 1 & 3 & 4 & 1 & 2 \\ 3 & 4 & 1 & 2 & 2 & 1 & 4 & 3 & 4 & 3 & 2 & 1\end{array}$

Definition 2.2 (Mutually Orthogonal Latin Squares). A set of Latin squares, $\mathcal{S}=\left\{S_{1}, \ldots, S_{k}\right\}$, is called a set of $k$ mutually orthogonal Latin 
squares if any pair of squares, $S_{\alpha}, S_{\beta} \in \mathcal{S}$, are orthogonal to each other. That is, no two pairs $\left(s_{\alpha_{i j}}, s_{\beta_{i j}}\right)$ and $\left(s_{\alpha_{l m}}, s_{\beta_{l m}}\right)$ are such that $\left(s_{\alpha_{i j}}, s_{\beta_{i j}}\right)=$ $\left(s_{\alpha_{l m}}, s_{\beta_{l m}}\right)$ unless $i=l$ and $j=m$.

A set of $k$ mutually orthogonal Latin squares of size $n$ are written as $k$ MOLS $n$. Theorem 2.3 gives $k \leq n-1$ as an upper bound for a $k M O L S$ $n$.

Theorem 2.3. There exist at most $(n-1) \operatorname{MOLS} n$ for $n \geq 1$

Proof. First note that it is possible without loss of generality to permute the entries of each square so that the first row is $1,2, \ldots, n$ and maintain the orthogonality between squares.

Assume the contrary, that there exist at least $n M O L S n$ and relabel the squares of $\mathcal{S}=\left\{S_{1}, S_{2}, \ldots, S_{n}\right\}$ so that first row of each square is $1,2, \ldots, n$.

Consider the entries in the cell of the first column and the second row of each $S_{i}$. The entries have to be distinct else $S_{\alpha_{21}}=S_{\beta_{21}}$ for some $S_{\alpha}, S_{\beta} \in \mathcal{S}$ $\alpha \neq \beta$. This would contradict the orthogonality of the squares since the entries of first row of $S_{\alpha}$ and $S_{\beta}$ give all pairs of the form $(x, x)$ for $x \in$ $\{1, \ldots, n\}$. Also, no $S_{i_{12}}=1$ since $S_{i}$ is a Latin square and $S_{i_{11}}=1$ for all $i \in\{1, \ldots, n\}$.

Thus a maximum $n-1$ mutually orthogonal Latin squares of order $n$ can exist.

A set of $k M O L S n$ is equivalent to an $O A(k+2, n)$ as shown in Theorem 2.4. Table 2.2 is an example of the implementation of Theorem 2.4 on the 3 MOLS 4 found in Table 2.1 .

Theorem 2.4. An $O A(k+2, n)$ exists if and only if $k$ MOLS $n$ exists. 
Proof. First assume an $O A(k+2, n)$ exists and note, that without loss of generality, it is possible to permute the columns of an $O A$ and maintain its properties.

By definition an $O A(k+2, n)$ has each pair $(x, y) x, y \in\{1,2, \ldots, n\}$ occurring in the first two positions of a column exactly once. This gives that for any $x$, them $n$ columns with $x$ in the first position and in these $n$ columns there is exactly one column that has a given element $y \in\{1,2, \ldots, n\}$ in the second position. Now permute the columns such that the first two elements of column, $c_{i}$, is such that $c_{i_{1}}=\left\lceil\frac{i}{n}\right\rceil$ and $c_{i_{2}} \equiv i+1 \bmod n$.

From the permuted $O A, k n \times n$ arrays are created. Let the arrays created be called $S_{1}, S_{2}, \ldots, S_{k}$. Row $j+2$ of the $O A$ contains the entries of the array $S_{j}$. The entry $s_{j_{\alpha \beta}}$ is $(j+2)$ entry in the column that has $\alpha$ in row 1 and $\beta$ in row 2. Essentially, the coordinates of the entries of $S_{j}$ are given by first two rows of the $O A$ and the entries themselves by the remaining rows.

We confirm that the set $\left\{S_{1}, S_{2}, \ldots, S_{k}\right\}$ of $n \times n$ are such that no $\left(s_{j_{\alpha \beta}}, s_{l_{\alpha \beta}}\right)=\left(s_{j_{\sigma \gamma}}, s_{l_{\sigma \gamma}}\right)$ for $j \neq l$ and $(\alpha, \beta) \neq(\sigma, \gamma)$. This is since $\left(s_{j_{\alpha \beta}}\right.$ ,$\left.s_{l_{\alpha \beta}}\right)$ and $\left(s_{j_{\sigma \gamma}}, s_{l_{\sigma \gamma}}\right)$ are pairs of entries in rows $j+2$ and $l+2$ from distinct columns of an $O A$. Also, in any individual square, $S_{j}$, no element occurs twice in an single row or column. If an element, $x$, is repeated in a row or column of $S_{j}$ then the entry $x$. is repeated in a column of the $O A$. But then the pair $(x, x)$ would be contained twice in the rows 1 and $j$ or 2 and $j$ contradicting the definition of an $O A$. With both these conditions met the set $\left\{S_{1}, S_{2}, \ldots, S_{k}\right\}$ of $n \times n$ arrays are a $k$ MOLS $n$.

Now an $O A(k+2, n)$ is formed from $k \operatorname{MOLS} n, \mathcal{S}=\left\{S_{1}, S_{2}, \ldots, S_{k}\right\}$. First a $k \times n^{2}$ array is formed from the $k \operatorname{MOLS} n$. The entry in row $\alpha$ and 
column $\beta$ of square $S_{j} \in \mathcal{S}$ is assigned to row $j$ and column $(\beta-1) * n+\alpha$ of the array. This gives which the ordered pairs that consist of column entries of two distinct rows of the $k \times n^{2}$ array correspond to $\left(S_{u_{\alpha \beta}}, S_{v_{\alpha \beta}}\right)$ for $S_{u}, S_{v} \in \mathcal{S}$. That is, every ordered pair from $X \times X$ occurs exactly once in every $2 \times n^{2}$ subarray of the $k \times n^{2}$ array and the $k \times n^{2}$ array is an $O A(k, n)$.

Two more rows can be added to extend the $k \times n^{2}$ array to an $O A(k+2, n)$. These two rows have the elements of column $c_{i}$, such that $c_{i_{1}}=\left\lceil\frac{i}{n}\right\rceil$ and $c_{i_{2}} \equiv i+1 \bmod n$. These rows contain every ordered pair between them and so it is only required to show that the orthogonal property holds between each of these rows and the rows of the $O A(k, n)$. The construction gives that every block of $n$ entries in positions $n(\psi-1)+1, n(\psi-1)+2, \ldots, n \psi$, for $\psi=1 \ldots n$, of a row corresponds to a row of a Latin square and thus contains every element $1,2, \ldots, n$. Hence, the entries of the first added row and any row from the $O A(k, n)$ produces every pair in $X \times X$. As well the $O A(k, n)$ from the $k$ $\operatorname{MOLS} n$ gives that the entries in positions $\psi, n+\psi, 2 n+\psi, \ldots,(n-1) n+\psi$ of a row correspond to a column of a Latin square and thus the entries contain every element $1,2, \ldots, n$. This gives that the entries from the second added row and any row from the $O A(k, n)$ contain all the pairs of $X \times X$. Thus when these two rows are concatenated with the $O A(k, n)$ an $O A(k+2, n)$ is formed. 
Table 2.2: An $O A(5,4)$ constructed via Theorem 2.4 with the 3 MOLS 4 from Table 2.1

$$
\left(\begin{array}{llllllllllllllll}
1 & 1 & 1 & 1 & 2 & 2 & 2 & 2 & 3 & 3 & 3 & 3 & 4 & 4 & 4 & 4 \\
1 & 2 & 3 & 4 & 1 & 2 & 3 & 4 & 1 & 2 & 3 & 4 & 1 & 2 & 3 & 4 \\
1 & 2 & 3 & 4 & 4 & 3 & 2 & 1 & 2 & 1 & 4 & 3 & 3 & 4 & 1 & 2 \\
1 & 2 & 3 & 4 & 3 & 4 & 1 & 2 & 4 & 3 & 2 & 1 & 2 & 1 & 4 & 3 \\
1 & 2 & 3 & 4 & 2 & 1 & 4 & 3 & 3 & 4 & 1 & 2 & 4 & 3 & 2 & 1
\end{array}\right)
$$

\subsection{Some Constructions for Orthogonal and Covering Arrays}

Many algebraic constructions exist for orthogonal arrays and equivalent designs. As well, there are many product constructions that form new arrays from existing ones. One of the most interesting constructions is when $v$ is a prime power. The construction depends on finite fields and a similar result was found by Mann [18]. Finite fields are known to exist if and only if $q=p^{n}$ for $p$ a prime.

Definition 2.5 (Finite Fields). A finite field is a triple $\mathbb{F}_{q}=(F,+, \times) . F$ is a finite set and + and $\times$ are binary operations on the set. Both operations are commutative with $a+b=b+a$ and $a \times b=b \times a \forall a, b \in F$. As well there are associative laws, $(a+b)+c=a+(b+c)$ and $(a \times b) \times c=a \times(b \times c)$ and distributive laws, $a \times(b+c)=a \times b+a \times c$ and $(a+b) \times c=a \times c+b \times c$ for all $a, b, c \in F$. There are also identity elements in $F: 0$ for + and 1 for $\times$. Inverses also exist: $a+(-a)=0=(-a)+a$ for all $a \in F$ and 
$a \times a^{-1}=0=a^{-1} \times a$ for all $a \in F a \neq 0$. Note that $a \times 0=0 \times a=0$ for all $a \in F$.

Theorem 2.6. For $q$ a prime power there exists an $O A(q+1, q)$ for $q \geq 2$

Proof. Let $\mathbb{F}_{q}$ be the finite field of order $q$. Define two $q \times 1$ arrays $v_{1}=$ $(1,1, \ldots, 1)^{T}$ and $v_{2}=\left(f_{1}, f_{2}, \ldots, f_{q}\right)^{T}$ where each $f_{i} \in \mathbb{F}_{q}$ are distinct.

Construct a $q \times q^{2}$ array, $A_{q}$, such that the columns are indexed by $\mathbb{F}_{q} \times \mathbb{F}_{q}$. The column $(i, j)$ is set to $i v_{1}+j v_{2}$.

For $A_{q}$ to be an $O A(q, q)$ each pair of distinct rows $x, y$ need to have a unique occurrence of all pairs $\left(f_{\alpha}, f_{\beta}\right) \in \mathbb{F}_{q} \times \mathbb{F}_{q}$. Since $A_{q}$ has $q^{2}$ columns then if every pair is unique then every pair occurs exactly once.

Let $x, y$ be a distinct pair of rows from $A_{q}, A_{q}(x,(i, j))=f_{\alpha}$, and $A_{q}(y,(i, j))=f_{\beta}$. The construction of $A_{q}$ gives that:

$$
\begin{aligned}
& f_{\alpha}=i+j f_{x}, \\
& f_{\beta}=i+j f_{y} .
\end{aligned}
$$

By subtracting the second equation from the first $f_{\alpha}-f_{\beta}=j\left(a_{x}-a_{y}\right)$. Since every element of $v_{2}$ is distinct $\left(a_{x}-a_{y}\right) \neq 0$. Thus there exists a $\left(a_{x}-a_{y}\right)^{-1} \in \mathbb{F}_{q}$, which gives:

$$
j=\left(a_{x}-a_{y}\right)^{-1}\left(f_{\alpha}-f_{\beta}\right) .
$$

Substituting the solution for $j$ into the first equations yields

$$
i=j a_{x}-f_{\alpha}=\left(a_{x}-a_{y}\right)^{-1}\left(f_{\alpha}-f_{\beta}\right) a_{x}-f_{\alpha}
$$

Since the solution to the system of equations is unique, each pair $\left(f_{\alpha}, f_{\beta}\right)$ is unique and $A_{q}$ is an $O A(q, q)$.

To form an $O A(q+1, q)$ concatenate $A_{q}$ with a row $u$ of length $q^{2}$ to form the array $A_{q+1}$. Index $u$ by $(i, j)$ and its entries are assigned such that $u_{i j}=j$ for all $j \in \mathbb{F}_{q}$. For $A_{q+1}$ to be an $O A(q+1, q)$ any given row of $A_{q}$, row $\gamma$, 
must be such that each $\left(\gamma_{i j}, u_{i j}\right)$ is distinct $\forall j_{0}=1, \ldots, q^{2}$. This would give all the possible $q^{2}$ pairs of $\mathbb{F}_{q} \times \mathbb{F}_{q}$.

Consider an arbitrary $j, j_{0}$, and row of $A_{q}, \gamma$. By definition $u_{i j_{0}}=j_{0}$ when $j=j_{0}$ for all $i \in F_{q}$. As well by definition $\gamma_{i, j_{0}}=i v_{1}+i v_{j_{0}}=i+v_{j_{0}}$. Thus each pair $\left(\gamma_{i j_{0}}, u_{i j_{0}}\right)=\left(i+v_{j_{0}}, j_{0}\right)$. Clearly $\left(i_{1}+v_{j_{0}}, j_{0}\right) \neq\left(i_{2}+v_{j_{0}}, j_{0}\right)$ for $i_{1}, i_{2} \in \mathbb{F}_{q}$ when $i_{1} \neq i_{2}$. This gives a set of $q$ distinct pairs of the form $\left(i_{2}+v_{j_{0}}, j_{0}\right)$. Since $j_{0}$ was chosen arbitrarily, all the sets of pairs, $\left(i_{2}+v_{j}, j\right)$ for all $j \in F_{q}$, gives all the $q^{2}$ distinct pairs. Since $\gamma$ was chosen arbitrarily this hold true for every row in $A_{q}$.

Thus this construction yields a $O A(q+1, q)$ for $q$ a prime power.

Theorem 2.7 gives a basic recursive construction for creating covering arrays. Theorem 2.7 and Theorem 2.6 combine to give MacNeish's Theorem 2.8. Many other constructions exist to build new covering arrays from existing ones $[8,10,24]$.

Theorem 2.7. Given $A_{1}=C A\left(N_{1} ; k, v_{1}\right)$ and $A_{2}=C A\left(N_{2} ; k, v_{2}\right)$ then there exists an $C A\left(N_{1} N_{2} ; k, v_{1} v_{2}\right)$.

Proof. Define a set $Y$ as $X_{1} \times X_{2}$, where $\left|X_{1}\right|=v_{1}$ and $\left|X_{2}\right|=v_{2}$, and an array, $A$, of size $k \times N_{1} N_{2}$. The columns of $A$ are indexed by $(i, j)$ where $i \in\left\{1, \ldots, N_{1}\right\}$ and $j \in\left\{1, \ldots, N_{2}\right\}$. For a row $\gamma$ of $A$ assign the entry $(i, j)$ as the pair $\left(a_{1_{\gamma i}}, a_{2_{\gamma j}}\right)$ where $a_{1_{\gamma i}}$ and $a_{2_{\gamma j}}$ are entries of $A_{1}$ and $A_{2}$ respectively.

Choose any two distinct rows, $\alpha, \beta$ of $A$ and an arbitrary pair $\left[\left(x_{1}, x_{2}\right),\left(\overline{x_{1}}, \overline{x_{2}}\right)\right] \in Y \times Y$. The pair $\left(x_{1}, \overline{x_{1}}\right)$ occurs in the rows $\alpha, \beta$ in exactly one column, $i_{0}$, of $A_{1}$. As well, the pair $\left(x_{2}, \overline{x_{2}}\right)$ occurs in the rows $\alpha$ and $\beta$ in 
exactly one column, $j_{0}$, of $A_{2}$. Thus there is exactly one column, $\left(i_{0}, j_{0}\right)$, of $A$ that has the pair $\left[\left(x_{1}, x_{2}\right),\left(\overline{x_{1}}, \overline{x_{2}}\right)\right]$. Thus $A$ is an $O A\left(N_{1} N_{2} ; k, v_{1} v_{2}\right)$

MacNeish's Theorem is given in Theorem 2.8.

Theorem 2.8 (MacNeish's Theorem). Given a prime power factorization of $n=p_{1}^{e_{1}} p_{2}^{e_{2}} \cdots p_{j}^{e_{j}}$ where each $p_{i}$ is a distinct prime and $e_{i} \geq 1$, then there exists an $O A(k+2, n)$ where $k=\min \left\{p_{i}^{e_{i}}-1 \mid i=1, \ldots, j\right\}$.

Proof. This is a corollary of the combination of the results of Theorem 2.6 and 2.7 .

\subsection{Permutation Groups and Difference De- signs}

The constructions in the previous section demonstrate abstract algebra can be a very useful tool in creating combinatorial designs. Permutation groups are used as a tool to create designs including orthogonal and covering arrays. A permutation group is a group that acts upon a set by a group action.

Definition 2.9 (Group Action). Let $G$ be a group and $X$ a nonempty set. Then an action of $G$ on a set $X$ is a map $\sigma_{g}(x): X \times G \rightarrow X$. The element $\sigma_{g}(x)$ is written as $x^{g}$. The map, $\sigma$, has the following properties:

i) $x^{1}=x$ for all $x \in X$ where 1 is the identity element of $G$, ii) $\left(x^{g}\right)^{h}=x^{g h}$ for all $g, h \in G$ and $x \in X$.

Every group action on $X$ is a subgroup of the symmetric group $S^{X}$, the symmetric group of order $|X|$. In this thesis the actions of the groups 
employed are regular, also referred to as sharply transitive, on a set $X$ or a subset of $X$. Fundamental concepts of permutation groups are needed to understand a regular group action.

Definition 2.10 (Orbit of an Element Under a Group Action). Given a group $G$ acting on a set $X$ then the orbit of a $x \in X$ is:

$x^{G}:=\left\{x^{g} \mid g \in G\right\}$.

A useful property of the orbits of a group action is that they form a partition of the set $X$.

Theorem 2.11. The orbit of $x, x^{G}$, and the orbit of $y, y^{G}$ are such that $x^{G}=y^{G}$ or $x^{G} \cap y^{G}=\emptyset$.

Proof. Choose an element, $z \in x^{G} \cap y^{G}$. By definition $z$ can be to sent any element in $x^{G}$ with some $g^{-1} \in G$. Likewise $z$ can be sent to any element in $y^{G}$ with some $h \in G$. Thus the group element $g h$ sends any chosen element of $x^{G}$ to any element of $y^{G}$. Hence, if $x^{G} \cap y^{G} \neq \emptyset$ then $x^{G}=y^{G}$.

Certain $g \in G$ may not affect a given element $x \in X$. These $g \in G$ comprise what is call the stabilizer of $x$ in $G$ and is written as:

$$
G_{x}:=\left\{g \in G \mid x^{g}=x\right\}
$$

A fundamental relation between the orbit of a $x \in X$ and its stabilizer in $G$, $G_{x}$, is stated by the Orbit Stabilizer Theorem 2.12.

Theorem 2.12 (Orbit Stabilizer Theorem ). Given a group $G$ acting on a set $X$ and $x \in X$ then $\left|x^{G}\right|\left|G_{x}\right|=|G|$. 
A transitive group action is one that has only one orbit, that is $x^{G}=X$. When the group action is transitive and $G_{x}=1$ the action is called regular. When $G$ is finite Theorem 2.12 gives that $|G|=|X|$.

Definition 2.13 (Regular Group Action). The action of $G$ on $X$ is regular when $x^{G}=X$ and $G_{x}=1$ for all $x \in X$.

A regular finite group action can be seen as the group acting on itself. All regular finite group actions are equivalent as shown in Lemma 2.14.

Lemma 2.14. Let $G$ and $H$ be finite groups that act regularly on the sets $X$ and $Y$ respectively. If there exists a group isomorphism, $\varphi$, between $G$ and $H$ then there exists a set isomorphism between $X$ and $Y$ such that the respective group actions are equivalent.

Proof. To show that the actions are equivalent, a set isomorphism $\sigma$, between $X$ and $Y$ must be found such that $x_{i}^{g}=x_{j}$ implies $\sigma\left(x_{i}\right)^{\varphi(g)}=\sigma\left(x_{j}\right)$ for all $x_{i}, x_{j} \in X, g \in G$.

Note that since $G$ and $H$ are finite groups that act regularly on $X$ and $Y$ there exists a unique $g \in G$ such that $x_{i}^{g}=x_{j}$ for all $x_{i}, x_{j} \in X$ and there exists a unique $h \in H$ such that $y_{i}^{h}=y_{j}$ for all $y_{i}, y_{j} \in Y$. As well since $\varphi$ is a group isomorphism, there exists a unique $g \in G$ such that $y_{i}^{\varphi(g)}=y_{j}$. Lastly note $|G|=|H|$ since $G$ and $H$ are isomorphic, and $|G|=|X|$ and $|H|=|Y|$ since the groups are finite and act regularly. Thus $|X|=|Y|$.

Now assign an index on $G$ such that

$$
G=\left\{g_{1}, g_{2}, \ldots, g_{|G|} \mid g_{1}=1\right\}
$$

Using this index on $G$ assign an index on $X$. First arbitrarily pick an $x_{1}$. Then let 


$$
X=\left\{x_{1}, x_{2}, \ldots, x_{|G|} \mid x_{i}=x_{1}^{g_{i}}\right\} .
$$

Using the group isomorphism, $\varphi$, and the index on $G$, assign an index on $Y$. First arbitrarily pick a $y_{1}$. Then let

$$
Y=\left\{y_{1}, y_{2}, \ldots, y_{|G|} \mid y_{i}=y_{1}^{\varphi\left(g_{i}\right)}\right\} .
$$

Now define a set isomorphism, $\sigma$, between $X$ and $Y$ such that $\sigma\left(x_{i}\right)=y_{i}$ for all $i \in\{1, \ldots,|G|\}$. It is now shown $x_{i}^{g}=x_{j}$ implies $\sigma\left(x_{i}\right)^{\varphi(g)}=\sigma\left(x_{j}\right)$ for all $x_{i}, x_{j} \in X, g \in G$. Chose an arbitrary $x_{i}, x_{j} \in X$. Then the set isomorphism $\sigma$ gives that $\sigma\left(x_{i}\right)=y_{i}$ and $\sigma\left(x_{j}\right)=y_{j}$. As well, by the way the groups and sets were indexed, $x_{i}^{g_{i}^{-1}}=x_{1}$ and $x_{1}^{g_{j}}=x_{j}$. Thus $g=g_{j} g_{i}^{-1}$.

Also, by definition $y_{i}^{\varphi\left(g_{i}^{-1}\right)}=y_{1}$ and $y_{1}^{\varphi\left(g_{j}\right)}=y_{j}$ implies $y_{i}^{\varphi\left(g_{j}\right) \varphi\left(g_{i}^{-1}\right)}=$ $y_{i}^{\varphi(g)}=y_{j}$.

Finally, $y_{i}^{\varphi(g)}=y_{j}$ implies $\sigma\left(x_{i}\right)^{\varphi(g)}=\sigma\left(x_{j}\right)$ by definition of $\sigma$. Thus the actions are equivalent.

Definition 2.15 (Difference Matrix). Let $G$ be a group acting on itself. Then $a(G, k, \lambda)$ difference matrix, $D$, is a $k \times g \lambda$ matrix such that for all $i \neq j$ the set $\left\{d_{i l} d_{j l}^{-1}, l \in\{1, \ldots,|G| \lambda\}\right\}$ contains every $g \in G \lambda$ times.

Definition 2.16 (Difference Covering Array). Let $G$ be a group acting on itself. Then a $(G, k, \lambda)$ difference covering array, $D$, is a size $k \times N$ matrix such that for all $i \neq j$ the set $\left\{d_{i l} d_{j l}^{-1}, l=\{1, \ldots, N\}\right\}$ contains every $g \in G$ at least $\lambda$ times.

Difference matrices and difference covering arrays can be used to construct orthogonal arrays and covering arrays, respectively. The group differences correspond to a partition of the 2-tuples of the set $G \times G$. The differences 
are equivalent to 2-tuple orbits, as shown in Theorem 2.17. The action of $G$ can send any pair to another pair in the same orbit. The next chapter shows, in great detail, how to make a covering array from an array that contains 2-tuple orbits.

Theorem 2.17. Let $G$ be a group and for all $g \in G d_{g}=\left\{\left(g_{\alpha}, g_{\beta}\right) \mid g=\right.$ $\left.g_{\alpha} g_{\beta}^{-1}\right\}$. Then $d_{g}$ is an orbit of the action of $G$ on the set $G \times G$.

Proof. If $d_{g}$ is an orbit of $G \times G$ then when $h$ acts on the pair the $\left(g_{\alpha}, g_{\beta}\right)$, $\left(g_{\alpha}, g_{\beta}\right)^{h} \in d_{g}$ for all $h \in G$.

A group acting on itself is a regular group action, all regular actions are equivalent by Lemma 2.14. Consider the action of right multiplication on a group difference. Now $\left(g_{\alpha}, g_{\beta}\right)^{h}=\left(g_{\alpha}^{h}, g_{\beta}^{h}\right)$ whose difference is $g_{\alpha} h\left(g_{\beta} h\right)^{-1}=$ $g_{\alpha} h h^{-1} g_{\beta}^{-1}=g_{\alpha} g_{\beta}^{-1}=g$.

Thus $\left(g_{\alpha}, g_{\beta}\right)^{h} \in d_{g}$ and $d_{g}$ is an orbit of the action of $G$ on $G \times G$. 


\section{Chapter 3}

\section{Background and}

\section{Techniques of the Constructions}

\subsection{Introduction}

This chapter details the structure used by the eight constructions employed in this thesis and outlines how the previous work is expanded by this thesis. Section 3.2 introduces the $\left[M^{G}, C\right]$ format developed by Chateauneuf, Colbourn, and Kreher [6] used to construct covering arrays. Section 3.3 describes how the $\left[M^{G}, C\right]$ format is employed to construct covering arrays with strength 2. Outlines are given in Section 3.4 of the two constructions that have been previously used by Meagher and Stevens, and Colbourn, Martirosyan, Mullen, Shasha, Sherwood, and Yucas [10, 19, 20, 21] to create least upper bounds on covering arrays and whose scope is broadened by this thesis. The two styles of construction shown are referred to as the Entire- $k$ single fixed point and Partial-k single fixed point. The last section of this chapter, 
Section 3.5, is devoted to a summary of the results of the previous implementations of the Entire- $k$ and Partial-k single fixed point constructions. Section 3.5 also provides an outline of how the previous study of cover starters for constructing strength 2 covering arrays is expanded by this thesis.

\subsection{Covering Orbit Representatives in Subarrays: The $\left[M^{G}, C\right]$ Structure}

The eight constructions used in this thesis use a common structure. This structure is an idea developed by Chateauneuf et al. [6] that they employed to construct strength 3 covering arrays. Four features are required for the construction: a set, $X$, of size $v$, a permutation group, $G$, that acts on $X$, and two arrays, $M$ and $C$, whose entries are from $X$. The size of $M$ is $k \times l$ and the size of $C$ is $k \times l_{c}$.

The array $\left[M^{G}, C\right]$ is formed by concatenating $C$ with arrays $\left\{M^{g_{1}}, M^{g_{2}}, \ldots, M^{g_{|G|}}\right\}$ which result from the action of each $g \in G$ on $M$. The elements of the subarray $M, m_{i j}$, are acted on by a $g \in G$ so that the element in row $i$ and column $j$ of $M^{g}$ is $m_{i j}^{g}$. There are $|G|$ such subarrays. The subarrays are concatenated horizontally together to form an array $M^{G}$

of size $k \times l|G|$. The array $M^{G}$ is then concatenated with the array $C$ horizontally to form $\left[M^{G}, C\right]$, an array of dimension $k \times\left(l|G|+l_{c}\right)$. Table 3.1 illustrates the $\left[M^{G}, C\right]$ format.

The central idea of the construction is that $M$ contains representatives from $t$-tuple orbits of the action of $G$ on $X$, where $t$ is the strength of the desired covering array. The action of $G$ produces orbits of $t$-tuples, $\mathcal{B}=$ 
Table 3.1: The concatenation of $\left[M^{G}, C\right]$ structure that is used to create covering arrays

$$
\left(M^{g_{1}}\left|M^{g_{2}}\right| M^{g_{3}}|\cdots| M^{g_{|G|}} \mid C\right)
$$

$\left\{B_{1}, B_{2}, \ldots, B_{b}\right\}$ which are a partition of the set of $t$-tuples (Theorem 2.11). For $M^{G}$ to cover an orbit, $B_{i}, M$ requires a $t$-tuple from $B_{i}$ in a column of every $t \times l$ subarray of $M$. If this condition on $M$ is met then $M^{G}$ covers all the $t$-tuples of $B_{i}$.

Lemma 3.1 (Orbit Covering Lemma). Let $X$ be a set, $G$ a permutation group that acts on $X$ and $B$ be an orbit of $t$-tuples from the action of $G$ on $X$. If $M$ is an array of size $k \times l$, and for all $t \times l$ subarrays of $M$ there exists a $t$-tuple from the orbit $B$ in some column, then all the $t$-tuples in $B$ are covered by $M^{G}$

Proof. Let $\left(x_{1}^{g_{\alpha}}, x_{2}^{g_{\alpha}}, \ldots, x_{t}^{g_{\alpha}}\right)$ be a given $t$-tuple from the orbit $B=$ $\left\{\left(x_{1}^{g_{1}}, x_{2}^{g_{1}}, \ldots, x_{\gamma}^{g_{1}}\right),\left(x_{1}^{g_{2}}, x_{2}^{g_{2}}, \ldots, x_{t}^{g_{2}}\right), \ldots,\left(x_{1}^{g_{|G|}}, x_{2}^{g_{|G|}}, \ldots, x_{t}^{g_{|G|}}\right)\right\}$. Let $U$ be a given $t \times l$ subarray of $M^{G}$. By definition $M$ has a $t$-tuple representative from the orbit $B,\left(x_{1}^{g_{\beta}}, x_{2}^{g_{\beta}}, \ldots, x_{t}^{g_{\beta}}\right)$, in a column of the $t \times l$ subarray of $M$ that has rows corresponding to rows of the subarray $U$.

Recall that the action of $G$ on $X$ gives that the entry from row $i$ and column $j$ of $M^{g}$ is $m_{i j}^{g}$ where $m_{i j}$ is the corresponding entry of $M$. Hence there is a column in $U$ of $M^{\left(g_{\beta}^{-1} g_{\alpha}\right)}$ where the $t$-tuple $\left(x_{1}^{g_{\alpha}}, x_{2}^{g_{\alpha}}, \ldots, x_{t}^{g_{\alpha}}\right)$ is found. Since the $t$-tuple and the $t \times l$ subarray, $U$, were taken in general and $M^{\left(g_{\beta}^{-1} g_{\alpha}\right)}$ is contained in $M^{G}$, there exists a column with any given $t$-tuple of 
$B$ in $M^{G}$.

The array $\left[M^{G}, C\right]$ covers $t$-tuples of orbits in two ways: either by $M^{G}$ or by $C$. The goal is to have $M^{G}$ cover all, or almost all, of the orbits so that $C$ is simple to construct and $l_{c}$ is small.

A $C A(33 ; 3,6,3)$ by Chateauneuf et al. [6] obtained from a $\left[M^{G}, C\right]$ construction is shown in Table 3.3. The covering array produced is a minimum covering array: $\operatorname{CAN}(3,6,3)=33$. The set is $X=\{0,1,2\}$, the group is the projective general linear group of order $3, G=P G L(3)$, and the number of factors, $k$, is 6 . The group, $G$, was chosen to have the property that it was sharply 3 -transitive on the set $X$. Table 3.2 illustrates 3 -tuple orbits of a sharply 3-transitive group. $M$ was constructed so that $M^{G}$ covers 3-tuples from the orbits $B_{2}, B_{3}, B_{4}$, and $B_{5}$. $C$ covers the remaining 3 -tuples, which are from $B_{1}$. In Table 3.3 the far right subarray is $C$ and the subarrays $M^{g}$ that result from $G$ acting on $M$ are the remaining partitioned subarrays.

Table 3.2: The 3-tuple orbits of a 3-transitive group .

$$
\begin{aligned}
& B_{1}(x, x, x): x \in X \\
& B_{2}(x, x, y): x, y \in X, x \neq y \\
& B_{3}(x, y, x): x, y \in X, x \neq y \\
& B_{4}(y, x, x): x, y \in X, x \neq y \\
& B_{5}(x, y, z): x, y, z \in X, x \neq y \neq z \neq x
\end{aligned}
$$

Similarly, strength 2 covering arrays can be developed from difference covering arrays. When a difference covering array, $M$, of size $k \times l$, exists 
Table 3.3: The $C A(33 ; 3,6,3)$ created by Chateauneuf et al. via the $\left[M^{G}, C\right]$ structure .

$$
\left(\begin{array}{llllll|lllll|lllll|llll|lllll|lllll|lll}
0 & 1 & 2 & 2 & 1 & 1 & 2 & 0 & 0 & 2 & 2 & 0 & 1 & 1 & 0 & 0 & 2 & 1 & 1 & 2 & 1 & 0 & 2 & 2 & 0 & 2 & 1 & 0 & 0 & 1 & 0 & 1 & 2 \\
1 & 2 & 2 & 1 & 0 & 2 & 0 & 0 & 2 & 1 & 0 & 1 & 1 & 0 & 2 & 2 & 1 & 1 & 2 & 0 & 0 & 2 & 2 & 0 & 1 & 1 & 0 & 0 & 1 & 2 & 0 & 1 & 2 \\
2 & 2 & 1 & 0 & 1 & 0 & 0 & 2 & 1 & 2 & 1 & 1 & 0 & 2 & 0 & 1 & 1 & 2 & 0 & 2 & 2 & 2 & 0 & 1 & 0 & 0 & 0 & 1 & 2 & 1 & 0 & 1 & 2 \\
2 & 1 & 0 & 1 & 2 & 0 & 2 & 1 & 2 & 0 & 1 & 0 & 2 & 0 & 1 & 1 & 2 & 0 & 2 & 1 & 2 & 0 & 1 & 0 & 2 & 0 & 1 & 2 & 1 & 0 & 0 & 1 & 2 \\
1 & 0 & 1 & 2 & 2 & 2 & 1 & 2 & 0 & 0 & 0 & 2 & 0 & 1 & 1 & 2 & 0 & 2 & 1 & 1 & 0 & 1 & 0 & 2 & 2 & 1 & 2 & 1 & 0 & 0 & 0 & 1 & 2 \\
0 & 0 & 0 & 0 & 0 & 1 & 1 & 1 & 1 & 1 & 2 & 2 & 2 & 2 & 2 & 0 & 0 & 0 & 0 & 0 & 1 & 1 & 1 & 1 & 1 & 2 & 2 & 2 & 2 & 2 & 0 & 1 & 2
\end{array}\right)
$$

for a group, $G$, then $M^{G}$ is a $C A(|G| l ; 2, k,|G|)$. By Theorem 2.17 the group differences are equivalent to the orbits of 2-tuples when a group acts on itself by right multiplication. Orbitals are another name for 2-tuple orbits and are used for the remainder of this thesis. Difference covering arrays are difficult to find $[30,31]$ and likewise an $M$ for a $\left[M^{G}, C\right]$ construction is hard to find.

\subsection{Cover Starter Generation of a Subarray}

Meagher and Stevens $[19,20,21]$ developed a way of simplifying the search for the initial array $M$. Searching for a valid $M$ has a search space of $v^{k l}$. They use a one dimensional array coined a starter vector. It is also called a cover starter by Colbourn et al. in [10] and this is the terminology used in this thesis. The cover starter is of size $\kappa \times 1$ and thus reduces the naive search space to $v^{\kappa}$. It is used to create a circulant array of size $\kappa \times \kappa$. Table 3.4 gives an example of a circulant array. Creating an $M$ as a $\kappa \times \kappa$ array from a cover starter significantly reduces the size of the object to use in the $\left[M^{G}, C\right]$ construction. The constructions examined herein, a $k \times 1$ or $(k-1) \times 1$ 
cover starter is found and then used to generate an $k \times l$ array for an $M$. The use of the $\left[M^{G}, C\right]$ algebraic structure and the search require to find the cover starters give that these constructions are a hybrid of algebraic and algorithmic search methods.

Definition 3.2 (Circulant Array). A circulant array, $\Phi$, is $a=\kappa \times \kappa$ array developed from $\tau=\left(x_{1}, \ldots, x_{\kappa}\right)^{T}$ such that $\Phi_{i j}=x_{z}, z \equiv(i-j+1) \bmod$ $\kappa$.

Table 3.4: A circulant array of size $\kappa \times \kappa$ formed from a $\kappa \times 1$ array $\left(x_{1}, \ldots, x_{\kappa}\right)^{T}$.

$$
\Phi=\left(\begin{array}{cccc}
x_{1} & x_{\kappa} & \ldots & x_{2} \\
x_{2} & x_{1} & \ldots & x_{3} \\
x_{3} & x_{2} & \ldots & x_{4} \\
\vdots & \vdots & \ddots & \vdots \\
x_{\kappa} & x_{\kappa-1} & \ldots & x_{1}
\end{array}\right)
$$

For a circulant array to satisfy the Orbit Covering Lemma 3.1 for an orbital, $B$, a cover starter requires a representative pair $\left(x_{i}, x_{j}\right)$ from $B$ at each distance $s$-apart $s=\{1, \ldots,(\kappa-1)\}$; a pair, $\left(x_{i}, x_{j}\right)$, in a $\kappa$-tuple, $\left(x_{1}, x_{2}, \ldots, x_{\kappa}\right)$, is $s$-apart if $(j-i) \equiv s \bmod \kappa-1$. Table 3.5 is an example of a $6 \times 6$ cyclic difference covering array developed from the group $\mathbb{Z}_{3}$ and the cover starter $(0,1,2,2,1,0)^{T}$.

Definition 3.3 (Cover Starter). Given a set $X$, a group $G$ acting on $X$, and a set of orbitals resulting from the action of $G$ on $X, \mathcal{B}=\left\{B_{1}, \ldots, B_{i}\right\}$, 
a cover starter is $a \kappa \times 1$ array that has entries from a set $X$ such that a representative pair of each orbital $B_{i} \in \mathcal{B}$ is at each distance s-apart for $s \in\{1, \ldots,(\kappa-1)\}$.

Lemma 3.4. Given a set $X$, a group, $G$, acting on $X$, a set of orbitals resulting from the action of $G$ on $X, \mathcal{B}=\left\{B_{1}, \ldots, B_{\gamma}\right\}$, and a length $\kappa$ cover starter, $T$, that contains a representative pair at each distance s-apart for $s \in\{1, \ldots,(\kappa-1)\}$ for each orbital $B_{i} \in B$ then the $\kappa \times \kappa$ circulant, $\Phi$, developed from $T$ contains some pair, $(x, y)$, from each orbital in $\mathcal{B}$ in each of its $2 \times \kappa$ subarrays.

Proof. Choose an arbitrary orbital $B$ from $\mathcal{B}$, and pair of rows, $i$ and $j$, from $\Phi$ such that $i \neq j$. By definition there exists some pair of entries $\left(t_{\alpha}, t_{\alpha+s}\right)$ of $\mathrm{T},\left(t_{\alpha}, t_{\alpha+s}\right)$ at distance $s$-apart where $s \equiv j-i \bmod k a p p a-1$. Let $\theta \equiv i-\alpha \bmod \kappa-1$. Then in column $\theta$ the pair $\left(t_{\alpha}, t_{\alpha+s}\right)$ occurs in the rows $(i, j)$.

Table 3.5: A circulant array developed from $(0,1,2,2,1,0)^{T}$ that is a difference covering array of size $6 \times 6$ over $\mathbb{Z}_{3}$.

$$
\left(\begin{array}{llllll}
0 & 1 & 2 & 2 & 1 & 0 \\
1 & 2 & 2 & 1 & 0 & 0 \\
2 & 2 & 1 & 0 & 0 & 1 \\
2 & 1 & 0 & 0 & 1 & 2 \\
1 & 0 & 0 & 1 & 2 & 2 \\
0 & 0 & 1 & 2 & 2 & 1
\end{array}\right)
$$


The number of columns in a $\left[M^{G}, C\right]$ construction is $N=l|G|+l_{c}$. The two most influential components of $N$ are $l$ and $|G|$. In Meagher and Stevens' original construction $l$ is fixed equal to $k$ since $M$ is a $k \times k$ circulant. Thus the size of the group $|G|$ is the main parameter influencing $N$ for their construction.

Meagher and Stevens discuss which types of group actions are efficient for the strength 2 covering arrays developed by their $\left[M^{G}, C\right]$ construction. They mention the Cauchy-Frobenius lemma, also known as Burnside's lemma, as a guide to finding the groups that give useful results $[19,20,21]$. Burnside's Lemma relates the number of orbitals, $b$, the number of ordered pairs that a $g \in G$ leaves fixed, $\mid$ fix $(g) \mid$, and the index of the group, $|G|$.

Lemma 3.5 (The Burnside's Lemma). $b|G|=\sum_{g \in G}|f i x(g)|$

From Burnside's Lemma, Meagher and Stevens were inspired to use group actions with large orbitals from small groups. They achieved this balance by choosing groups of size $|G|=v-1$. The groups act regularly on $v-1$ points and fix the remaining point. For simplicity they only used cyclic groups $[19,20,21]$.

The fixed point of the group is denoted as $\infty$ and the rest of the elements are an embedding of $G$ into $X \backslash\{\infty\}$. The set is labelled as $X=\left\{\infty, g_{1}, g_{2}, \ldots, g_{|G|}\right\}$. The initial array, $M$, is of size $k \times k$ as it is a circulant developed from a $k \times 1$ cover starter. It can be considered a difference covering array over the elements $X \backslash\{\infty\}$ that also contains the orbitals of the form $\left(\infty, g_{i}\right)$ and $\left(g_{i}, \infty\right)$ for every $2 \times k$ subarray of $M . C$ is a $k \times 1$ array with $\infty$ in every position and accounts for only remaining orbital, $\{(\infty, \infty)\}$. There are four constructions used in this thesis with a 
length $k$ cover starter, i.e. $\kappa=k$. They are denoted as an Entire- $k$ style of construction.

This version of a $\left[M^{G}, C\right]$ produces a $C A((v-1) k+1 ; k, v)$. The naive lower bound for $k$ is $v+1$. This bound is explained in Subsection 4.3.2 When $k=v+1$ the resulting covering array, $C A\left(v^{2} ;(v+1), v\right)$, is an orthogonal array. This demonstrates that the covering arrays built by this construction, for cover starters found close to the lower bound on $k$, are efficient.

Colbourn et al. use a similar $\left[M^{G}, C\right]$ construction to develop covering arrays with the same parameters [10]. The construction has the same group actions as Meagher and Stevens and thus $M^{G}$ and $C$ must account for the same orbitals. Again, the initial array, $M$, is a difference covering array over the elements $X \backslash\{\infty\}$ that also contains the orbitals of the form $\left(\infty, g_{i}\right)$ and $\left(g_{i}, \infty\right)$ for every $2 \times k$ subarray of $M$. The $k \times 1$ array with $\infty$ in every position, $C$, accounts for only remaining orbital, $(\infty, \infty)$. The difference is how $M$ is formed.

Definition 3.6 (Distinct Cover Starter). Given a set $X$, a group $G$ acting on $X$, and a set of orbitals resulting from the action of $G$ on $X$, $\mathcal{B}=\left\{B_{1}, \ldots, B_{i}\right\}, a$ distinct cover starter is a $\kappa \times 1$ array that has entries from a set $X$ such that a representative pair of each orbital $B_{i} \in \mathcal{B}$ is at each distance $s$-apart for $s \in\{1, \ldots,(\kappa-1)\}$ and each element $x \in X$ is contained somewhere in the distinct cover starter.

In the Colbourn et al. variation a length $k-1$ cover starter is employed. The four constructions analyzed in this thesis that use a length $k-1$ cover starter are termed Partial- $k$ style constructions. This construction style requires a distinct cover starter. The cover starter is required to contain a 
representative of all the orbitals at each distance apart except for the orbitals with pairs of the form $\left\{\left(g_{i}, g_{i}\right)^{G}\right\}$ and $\{(\infty, \infty)\}$. A $(k-1) \times(k-1)$ circulant subarray is developed from the cover starter. $M$ is formed by first concatenating this circulant subarray vertically with a $1 \times(k-1)$ array with $g_{1} \in X$ in every entry; the element $g_{1}$ is arbitrarily chosen. Next, this $k \times(k-1)$ array is concatenated horizontally with a $k \times 1$ array that has $\infty$ in $k^{\text {th }}$ position and $g_{1}$ in the remaining entries. Table 3.6 illustrates the resulting $M$.

Table 3.6: The $M$ of the Partial- $k$ single fixed point construction formed from a distinct cover starter.

$$
\left(\begin{array}{ccccc}
x_{1} & x_{k} & \ldots & x_{2} & g_{1} \\
x_{2} & x_{1} & \ldots & x_{3} & g_{1} \\
x_{3} & x_{2} & \ldots & x_{4} & g_{1} \\
\vdots & \vdots & \ddots & \vdots & \ldots \\
x_{k-1} & x_{k-2} & \ldots & x_{1} & g_{1} \\
g_{1} & g_{1} & \ldots & g_{1} & \infty
\end{array}\right)
$$

The naive lower bound on the size of $M$ is still $(v+1) \times(v+1)$, which is explained in Subsection 4.3.3. As with the Entire- $k$ construction, covering arrays built meeting this bound are orthogonal arrays. 


\subsection{Analysis of Past Results}

Meagher and Stevens, and Colbourn et al. respectively used Entire- $k$ and Partial- $k$ styles of single fixed point constructions with cyclic groups to find covering arrays, $C A(k(v-1)+1 ; k, v)[10,19,20,21]$. New least upper bounds for covering arrays were obtained and are noted in Table 3.7 and the existence results from Meagher and Stevens exhaustive searches for cyclic groups with $|G| \leq 9$ are given in Table 3.8. These bounds are used to achieve new least upper bounds on higher strength covering arrays as well $[10,21]$. In general, the smaller the value of $k$ by a construction, the more likely the covering array produces a new bound. When $k$ is relatively high compared to $v$ other techniques overtake the cover starter style constructions in efficiency [21]. For this reason the upper bound of the existence of cover starters is not explored and the existences of $k$ are only exhaustively searched for near the lower bound of $k$ for a given $v$ and $G$.

Table 3.7: Parameters where Meagher and Stevens' Entire- $k$, and Colbourn et al.'s Partial- $k$ single fixed point constructions yielded new least upper bounds on $C A N_{1}(2, k, v)[10,19,20,21]$

\begin{tabular}{|c|c|}
\hline$v$ & $k$ \\
\hline 3 & 5 \\
4 & 6 \\
5 & 7,8 \\
6 & 9,10 \\
7 & $10-12$ \\
8 & $11-15$ \\
\hline
\end{tabular}

\begin{tabular}{|c|c|}
\hline$v$ & $k$ \\
\hline 9 & $13-18$ \\
10 & $15-19$ \\
11 & $16-19$ \\
12 & $18-24$ \\
13 & $21-26$ \\
\hline
\end{tabular}

\begin{tabular}{|c|c|}
\hline$v$ & $k$ \\
\hline 14 & $23-32$ \\
15 & $26-30$ \\
16 & $28-32$ \\
17 & $31-42$ \\
18 & $33-38$ \\
\hline
\end{tabular}


Two questions that arise from the results produced by Meagher and Stevens' results for the Entire- $k$ single fixed point cover starters using cyclic groups:

- What causes gaps in the spectra of $k$ ? Is the spectra determined by group employed? The exhaustive search results given in Table 3.8 indicate a gap in the spectra of $k$ for $v=8$ when $k=10$.

- Can a cover starter of length $\kappa+1$ be formed from one of length $\kappa$ ? In Table 3.9 this discontinuity is contrasted by two sets of cover starters of length $k+1$ that appear to directly follow from a starter of length $k$.

Previously no behavioural analysis of cover starter existences was given for the Entire- $k$ or Partial- $k$ single fixed point constructions. Note that the errors in previously recorded values for $v=4$ have been corrected in this thesis: the values of $k$ for which a cover starter by $\mathbb{Z}_{2}$ for the Entire- $k$ single fixed point construction where $v=3 \mathrm{e}$ is listed as $5,8[10,19,20,21]$ and the updated values for $k$ are $5-8$. There are no reported exhaustive search results by Colbourn et al. for the Partial- $k$ single fixed point construction by cyclic groups; only parameters that yield new least upper bound were mentioned.

This thesis analyzes the spectra of $k$ and obtains new least upper bounds by expanding the knowledge of cover starter constructions of strength 2 covering arrays in the three following ways:

- By expanding the exhaustive and heuristic search results of the Entire- $k$ and Partial- $k$ single fixed point construction. 
Table 3.8: Previously Found spectra of $k$ by Meagher and Stevens' exhaustive searches for the Entire- $k$ single fixed point construction [21].

\begin{tabular}{|c|c|c|}
\hline$v$ & Group & Existence Spectra of $k$ \\
\hline \hline 3 & $\mathbb{Z}_{2}$ & $5-8$ \\
\hline 4 & $\mathbb{Z}_{3}$ & $5-10$ \\
\hline 5 & $\mathbb{Z}_{4}$ & $7-12$ \\
\hline 6 & $\mathbb{Z}_{5}$ & $9-14$ \\
\hline 7 & $\mathbb{Z}_{6}$ & $10-16$ \\
\hline 8 & $\mathbb{Z}_{7}$ & $9,11-18$ \\
\hline 9 & $\mathbb{Z}_{8}$ & $13-20$ \\
\hline 10 & $\mathbb{Z}_{9}$ & $15-22$ \\
\hline
\end{tabular}

- With six new cover starter constructions of covering arrays by Entire- $k$ and Partial- $k$ styles for no fixed point and double fixed point group actions.

- For the first time, in the case of all eight constructions, cover starters for all groups of a given order are search for instead of just the cyclic groups.

This thesis expands the exhaustive searches of Meagher and Stevens for the Entire- $k$ single fixed point construction and for the first time performs exhaustive searches for the Partial- $k$ single fixed point construction. The proofs of correctness of these two constructions are given in Section 4.3. Also note that for the first time non-cyclic groups are employed. These alternate groups' cover starters are exhaustively searched in same ranges that the cyclic groups are searched.

The six new constructions are introduced in Sections 4.2 and 4.4. There 
Table 3.9: Selected cover starters found by Meagher and Stevens for the Entire- $k$ single fixed point construction [21]

\begin{tabular}{|c|c|c|r|}
\hline$v$ & $G$ & $k$ & \\
\hline \hline 8 & $Z_{7}$ & 13 & $\infty 000010265023$ \\
& & 14 & $\infty 0000010265023$ \\
& & 15 & $\infty 00000010265023$ \\
\hline 11 & $Z_{10}$ & 18 & $\infty 00000014720295471$ \\
& & 19 & $\infty 000000014720295471$ \\
& & 20 & $\infty 0000000014720295471$ \\
& & 21 & $\infty 00000000014720295471$ \\
& & 22 & $\infty 000000000014720295471$ \\
& & 23 & $\infty 0000000000014720295471$ \\
\hline
\end{tabular}

is an Entire- $k$ and a Partial- $k$ version given for each of a no fixed point, simple double fixed point, and augmented double fixed point constructions. All groups of an appropriate order are used to find cover starters for a given $v$. These constructions are exhaustively searched for when values of $v$ and $k$ are feasible and in most cases heuristics are used to search beyond the exhaustive limits. The details of the search methods used for all the constructions are given in Section 4.5.

Analysis of the spectra of $k$ in Chapter 5 explains much of the spectra's behaviour that was previously unresolved. New least upper bounds on strength 2 covering arrays are produced by the work of this thesis via expanded searches, the use of non-cyclic groups, and the use of new constructions. 


\section{Chapter 4}

\section{The Constructions and Search Methods}

\subsection{Introduction}

In this chapter the details and proofs of the eight covering array constructions are given and the search methods are explained. Six of the constructions are new and the two single fixed point constructions have been previously used $[10,19,20,21]$. In this section an outline of the $\left[M^{G}, C\right]$ format as employed by the eight constructions is given and the orbital types as seen in Table 4.1 are defined. Section 4.5 describes the searches. Sections $4.2-4$ are each dedicated to the constructions of a like number of fixed points. There is a construction of the Entire- $k$ style and the Partial- $k$ style for each of the no fixed point, single fixed point, simple double fixed point, and augmented double fixed point. The introduction of each of these sections describes the similarities between the constructions and searches used for a given number 
of fixed points. Each of these three sections have a Entire- $k$ and Partial- $k$ subsection that detail the bounds and proofs for the constructions of that style. All the constructions are of the $\left[M^{G}, C\right]$ format as laid out in the Chapter 3.

The set size, $|X|=v$, is equal to $|G|$ for the no fixed point, $|G|+1$ for the single fixed point, and $|G|+2$ for the double fixed point constructions. The fixed points are denoted as $\infty$ for the single fixed point cases and $\left\{\infty_{1}, \infty_{2}\right\}$ in the double fixed point cases. The number of factors, $k$, has its own specific naive lower bound relative to $v$ for given construction but in all cases $k \geq v$.

The group action on $X$ produces a set of orbitals that are partitioned into orbital types in Table 4.1 to simplify the proofs of the constructions. For all the eight constructions $M^{G}$ covers the pairs of the Type II, III and IV orbitals and $C$ covers the pairs of the Type I orbitals.

Table 4.1: The orbital types of the group action of $G$ on $X=\left\{g_{1}, \ldots, g_{|G|}\right\} \cup$ $\left\{\infty_{1}, \ldots, \infty_{\rho}\right\}$ when $G$ acts regularly on $\left\{g_{1}, \ldots, g_{|G|}\right\}$ and fixes $\left\{\infty_{1}, \ldots \infty_{\rho}.\right\}$

$$
\begin{aligned}
& \text { Type I }\left(\infty_{i}, \infty_{j}\right) \\
& \text { Type II }\left(\infty_{i}, g_{j}\right) \text { and }\left(g_{j}, \infty_{i}\right) \\
& \text { Type III }\left(g_{i}, g_{i}\right) \\
& \text { Type IV }\left(g_{i}, g_{j}\right) i \neq j
\end{aligned}
$$

The number of Type I and II orbitals depend on the number of fixed points. The number of Type I orbitals is simply the square of the fixed points, $(v-|G|)^{2}$. The number of orbitals of Type II is two times the number 
of fixed points, $2(v-|G|)$. The is always one Type III orbital. The Type IV orbitals depend on the size of the group. There are $|G|^{2}$ total pairs of the set $G \times G$. Thus there are $|G|^{2}$ total pairs for all the type III and IV orbitals. Each orbit of a $g \in G$ for the group $G$ acting regularly on the set $G$ is of length $|G|$. Thus the total number of Type III and IV orbitals is $|G|$. There is one Type III orbitals leaving $|G|-1$ Type IV orbitals.

The $\left[M^{G}, C\right]$ structure as it is employed by the eight constructions is given now. The group $G$ acts regularly on $|G|$ elements of $X$ and fixes the remaining $v-|G|$. An array, $M$, of size $k \times l$ has entries from $X$. The $M$ contains in every $2 \times l$ subarray a representative pair of all the Type II, III, and IV orbitals. For every element $g \in G$ there is a corresponding array $M^{g}$, whose entries are simply $m_{i j}^{g}$, where is $m_{i j}$ is the $i j^{t h}$ entry of $M$. The $|G|$ resulting arrays are concatenated horizontally to form the array $M^{G}$ of size $k \times|G| l$. By Lemma 3.1 $M^{G}$ covers all the pairs of Type II, III, and IV orbitals. For six of the constructions another array another array $C$ of size $k \times l_{c}$ to form $\left[M^{G}, C\right] . C$ covers the type I orbitals.

\subsection{No Fixed Points}

\subsubsection{Introduction and the Search Reductions}

In the no fixed point case there is a set $X=\left\{g_{1}, \ldots, g_{|G|}\right\}$ and a group $G$ that acts regularly on $X$. This gives that $|G|=|X|$ by Theorem 2.12. Since there are no fixed points there no Type I orbitals and no subarray $C$ is needed. Thus covering array is formed by $M^{G}$ alone. Resulting from this group action are $|G|$ orbitals of Type III and IV. The form of these orbital types are given 
in Table 4.2. There is one orbital of type III, $B_{1}$, that is of the form $(x, x)$. The other $|G|-1$ orbitals are type IV of the form $(x, y)$ where $x \neq y$.

Table 4.2: Orbital Types Resulting From Group Acting on Itself.

$$
\begin{aligned}
& \text { Type III }\left(g_{i}, g_{i}\right): g_{i} \in X \\
& \text { Type IV }\left(g_{i}, g_{j}\right): g_{i}, g_{j} \in X, i \neq j
\end{aligned}
$$

Table 4.3 gives a concrete example of the resulting orbitals with $X=$ $\{0,1,2,3\}$ and $G$ an embedding of $\mathbb{Z}_{4}$. The elements of $X$ correspond to addition modulo four.

Table 4.3: Orbitals resulting from a the group $\mathbb{Z}_{4}$ acting on itself by left multiplication.

$$
\begin{aligned}
& B_{1}=\{(0,0),(1,1),(2,2),(3,3)\} \\
& B_{2}=\{(0,1),(1,2),(2,3),(3,0)\} \\
& B_{3}=\{(0,2),(1,3),(2,0),(3,1)\} \\
& B_{4}=\{(0,3),(1,0),(2,1),(3,2)\}
\end{aligned}
$$

The array $M$ is of size $k \times k$ and $|G|=v$. Thus the number of columns of the resulting covering array is $N=v k$. Also, since the orbitals are only comprised of embedded group elements, the resulting $M$ is a difference covering array.

The exhaustive searches fix the first element of $T$ without loss of generality by Theorem 4.1. This gives the number of $\kappa$-tuples needed to be searched as $v^{\kappa-1}$. 
Theorem 4.1. Let $X$ be a set and $G$ be a group that acts on regularly on $|G|$ elements of $X$ and fixes the rest. Let $T$ be a valid $\kappa \times 1$ cover starter. Then $T^{g}$ for all $g \in G$ also a valid cover starter.

Proof. Assume the contrary: that is there exists $g \in G$ such that for a distance, $d, T^{g}$ has no representative of an orbital $B$. By definition there exists a pair $\left(t_{i}, t_{j}\right)$ such that $(j-i) \equiv d \bmod \kappa$. This gives that $\left(t_{i}^{g}, t_{j}^{g}\right)$ would be $d$-apart in $T^{g}$ and $\left(t_{i}^{g}, t_{j}^{g}\right)$ is in the pair orbital $B$. Hence a contradiction on the assumption and the theorem is proven.

\subsubsection{Entire- $k$ Style Construction}

The cover starter for this construction is a $1 \times k$ array that contains a representative pair of all the orbitals at the distances $s$-apart for $s=1, \ldots,(k-1)$. The form of the array $M$ is shown in Table 4.4 and Table 4.5 is an example of a $C A(20 ; 5,4)$ by the Entire- $k$ no fixed point construction with $G=\mathbb{Z}_{4}$.

The lower bound on $k$ relative to $v$ of this construction is dictated by the number of orbitals that the cover starter $T$ has to contain. There are $|G|$ orbitals in total and hence $k$ must be at least $|G| \times 1$ to account for every orbital at distance apart. Since $v=|G|, k \geq v$.

Theorem 4.2. [Entire- $k$ No Fixed Point Construction] Let $X$ be a set where $|X|=v$ and $G$ be a group where $v=|G|$ and $G$ acts regularly on $X$. Let $T$ be a cover starter of size $k \times 1$ that covers all the orbitals $\mathcal{B}=\left\{B_{1}, B_{2}, \ldots, B_{|G|}\right\}$ at each distance $s$-apart for $s=1,2, \ldots,(k-1)$. If $M$ is the circulant array of $T$, then $M^{G}$ is a covering array, $C A(k v ; k, v)$. 
Table 4.4: The $M$ of the Entire- $k$ constructions.

$$
\left(\begin{array}{cccc}
x_{1} & x_{k} & \ldots & x_{2} \\
x_{2} & x_{1} & \ldots & x_{3} \\
x_{3} & x_{2} & \ldots & x_{4} \\
\vdots & \vdots & \ddots & \vdots \\
x_{k} & x_{k-1} & \ldots & x_{1}
\end{array}\right)
$$

Table 4.5: A Entire- $k C A(20 ; 5,4)$ with No Fixed Points with the Group $G=\mathbb{Z}_{4}$

$$
\left(\begin{array}{lllll|lllll|lllll|lllll}
0 & 2 & 1 & 2 & 0 & 1 & 3 & 2 & 3 & 1 & 2 & 0 & 3 & 0 & 2 & 3 & 1 & 0 & 1 & 3 \\
0 & 0 & 2 & 1 & 2 & 1 & 1 & 3 & 2 & 3 & 2 & 2 & 0 & 3 & 0 & 3 & 3 & 1 & 0 & 1 \\
2 & 0 & 0 & 2 & 1 & 3 & 1 & 1 & 3 & 2 & 0 & 2 & 2 & 0 & 3 & 1 & 3 & 3 & 1 & 0 \\
1 & 2 & 0 & 0 & 2 & 2 & 3 & 1 & 1 & 3 & 3 & 0 & 2 & 2 & 0 & 0 & 1 & 3 & 3 & 1 \\
2 & 1 & 2 & 0 & 0 & 3 & 2 & 3 & 1 & 1 & 0 & 3 & 0 & 2 & 2 & 1 & 0 & 1 & 3 & 3
\end{array}\right)
$$

Proof. Since the Entire- $k$ no fixed point cover starter contains a representative pair of every $B_{i} \in \mathcal{B}$, Lemma 3.4 and the Orbit Covering Lemma 3.1 give that $M^{G}$ is a covering array.

\subsubsection{Partial- $k$ Style Construction}

The array $M$ is adapted from the Partial- $k$ single fixed point construction and its form shown Table 4.6. The type III orbitals are accounted for by the last column of $M$. The type IV orbitals are accounted for by the first $(k-1)$

columns. The cover starter, $T$, must contain all the elements of $X \backslash\left\{g_{i}\right\}$ 
for an arbitrarily chosen $g_{i}$. In the examples $g_{1}$ is chosen. An example of a $C A(20 ; 5,4)$ by this construction is given in Table 4.7 where $|G|=\mathbb{Z}_{4}$.

The lower limit on the $k$ with respect to $v$ is determined independently by two requirements. One is the number of orbitals that the cover starter must contain. This is the number of type IV orbitals, $|G|-1$. This gives that $T$ must be at least of length $|G|-1$, since $v-1=|G|-1, k \geq v$. Also, the cover starter must contain $|G|-1$ unique elements, which independently gives the same bound on $k: k \geq|G|=v$.

Table 4.6: The Array $M$ of the Partial- $k$ no fixed point construction

$$
\left(\begin{array}{ccccc}
x_{1} & x_{k-1} & \ldots & x_{2} & g_{1} \\
x_{2} & x_{1} & \ldots & x_{3} & g_{1} \\
x_{3} & x_{2} & \ldots & x_{4} & g_{1} \\
\vdots & \vdots & \ddots & \vdots & g_{1} \\
x_{k-1} & x_{k-2} & \ldots & x_{1} & g_{1} \\
g_{1} & g_{1} & \ldots & g_{1} & g_{1}
\end{array}\right)
$$

Table 4.7: A Partial- $k$ no fixed points $C A(20 ; 5,4)$ with the group $G=\mathbb{Z}_{4}$

$$
\left(\begin{array}{lllll|lllll|lllll|lllll}
0 & 2 & 3 & 1 & 0 & 1 & 3 & 0 & 0 & 1 & 2 & 0 & 1 & 3 & 2 & 3 & 1 & 2 & 0 & 3 \\
1 & 0 & 2 & 3 & 0 & 2 & 1 & 3 & 3 & 1 & 3 & 2 & 0 & 1 & 3 & 0 & 3 & 1 & 2 & 3 \\
3 & 1 & 0 & 2 & 0 & 0 & 2 & 1 & 1 & 1 & 1 & 3 & 2 & 0 & 1 & 2 & 0 & 3 & 1 & 3 \\
2 & 3 & 1 & 0 & 0 & 3 & 0 & 2 & 1 & 1 & 0 & 1 & 3 & 2 & 0 & 1 & 2 & 0 & 3 & 3 \\
0 & 0 & 0 & 0 & 0 & 1 & 1 & 1 & 1 & 1 & 2 & 2 & 2 & 2 & 2 & 3 & 3 & 3 & 3 & 3
\end{array}\right)
$$


Theorem 4.3. [Partial-k No Fixed Point Construction] Let $X$ be a set where $|X|=v$ and $G$ be a group that acts regularly on $X$. Let the orbitals be $\mathcal{B}=\left\{B_{1}, B_{2}, \ldots, B_{|G|}\right\}$ and the orbital $B_{1}$ be the type III orbital. Let $T$ be a cover starter of size $(k-1) \times 1$ that covers all the type IV orbitals at every $s$-apart distance, $s=1,2, \ldots,(k-2)$ and also has every element from the set $\left\{g_{2}, g_{3}, \ldots, g_{|G|}\right\}$ in some position. Let $M$ be of the Partial-k no fixed point construction. Then $M^{G}$ is a covering array $C A(k v ; k, v)$.

Proof. There are no fixed points and thus only the orbitals of type III and IV need to be covered. $M^{G}$ covers the pairs of of $X \times X$ in 3 cases.

Case 1: Since the Partial- $k$ no fixed point cover starter contains a representative pair of every Type IV orbital, Lemma 3.4 gives there is a representative pair for every Type IV orbit in each pair of rows $i, j$ for all $i, j=1, \ldots, k-1$ and $i \neq j$.

Case 2: Let $(x, y)$ be a pair from a type III or IV orbital with one element from row $k$ in $M$ and the other in a row $1, \ldots,(k-1)$. By construction $g_{1}$ is placed in row $k$ of $M$ from the first $k-1$ columns and $T$ has every element from $\left\{g_{1}, g_{2}, \ldots, g_{|G|}\right\}$ in some position. The circulant array of $T$ is a subarray that comprises the first $(k-1)$ rows and columns of $M$. Thus there exists a column, $j$, of $M$ where $g_{1}$ is in row $k$ with $g_{i} \forall\left\{g_{1}, \ldots, g_{|G|}\right\}$ in a given row of the first $(k-1)$ rows. Consider the pair $\left(g_{1}, g_{\alpha}\right)$ and $\left(g_{1}, g_{\beta}\right), \alpha \neq \beta$ cannot be in the same orbital. Thus by the pigeon hole principle all the orbitals of type III and IV have a representative at every distance apart when one element of the pair is in row $k$.

Case 3: Let $(x, x)$ be a pair from the type III orbital. In $M$ every entry in the final column is $g_{1}$. Hence, for all rows $i, j$ with $i, j$ from $1, \ldots, k$ the 
pair $\left(g_{1}, g_{1}\right)$ represents the type III orbital.

The three cases give that $M$ has a representative pair of each orbital of the group action in every $2 \times \kappa$ subarray of $M$ and thus by the Orbit Covering Lemma 3.1 the Partial- $k$ no fixed point construction produces a covering array.

\subsection{Single Fixed Point}

\subsubsection{Introduction and the Search Reductions}

The single fixed point construction is the original of both the Entire- $k$ and Partial- $k$ styles $[10,21]$. Here $X=\left\{\infty, g_{1}, g_{2}, \ldots, g_{|G|}\right\}$. The fixed point is $\infty$ and $\left\{g_{1}, g_{2}, \ldots, g_{|G|}\right\}$ is the embedding of $G$ into $X \backslash\{\infty\}$. All four of the types of orbitals are present when $G$ acts on $X$.

In total there are $v+2$ orbitals. There is one orbital of type I, two of type II, one type III, and $|G|-1$ type IV orbitals. Table 4.8 is an example of the orbitals that result when the group used is $\mathbb{Z}_{4}$. In the example $X=$ $\{\infty, 0,1,2,3\}$.

For the single fixed point constructions the array $M^{G}$ covers the type II, III, and IV orbitals and the array $C$ covers the type I orbitals. $M$ is of size $k \times k$. C is the same for both constructions and is simply a column of the fixed element, $\infty$. Thus the covering array produced has $N=k \cdot(v-1)+1$ columns.

Efficiencies are utilized to reduce the size of the search space of the exhaustive search for the single fixed point cover starters. Theorem 4.4 gives that if a single fixed point cover starter exists, then a cover starter with a sin- 
Table 4.8: Orbitals Resulting From The Group $\mathbb{Z}_{4}$ Acting Regularly on $X \backslash$ $\{\infty\}$ and Fixed $\infty$

$$
\begin{aligned}
& B_{1}=\{(\infty, \infty)\} \\
& B_{2}=\{(\infty, 0),(\infty, 1),(\infty, 2),(\infty, 3)\} \\
& B_{3}=\{(0, \infty),(1, \infty),(2, \infty),(3, \infty)\} \\
& B_{4}=\{(0,0),(1,1),(2,2),(3,3)\} \\
& B_{5}=\{(0,1),(1,2),(2,3),(3,0)\} \\
& B_{6}=\{(0,2),(1,3),(2,0),(3,1)\} \\
& B_{7}=\{(0,3),(1,0),(2,1),(3,2)\}
\end{aligned}
$$

gle occurrence of the fixed point can be formed from the known starter. The starter is formed only by elements from $X \backslash\{\infty\}$ in the other $k-1$ positions. Theorem 4.1 and 4.4 combine to allow one to fix the two positions of $T$ for the exhaustive searches. That is one position as the fixed point and one as a chosen $g_{i}$. The exhaustive search can then be limited to checking $(v-1)^{\kappa-2}$ starters. Note that Theorem 4.4 is written specifically for the Entire- $k$ single fixed point cover starter but the analog for the Partial- $k$ starter is provided when the type III orbitals are omitted from the theorem and proof.

Theorem 4.4. Let $X=\left\{\infty, g_{1}, g_{2}, \ldots, g_{|G|}\right\}$ and $G$ act regularly on $X \backslash\{\infty\}$. Let $T$ be a $\kappa \times 1$ array containing representative pairs at each distance s-apart for $s=1,2, \ldots,(\kappa-1)$ for all the orbitals of type II, III, and IV. Create $T_{0}$ by replacing all but one arbitrary occurrence of the fixed point, $\infty$, in $T$ with some $g \in X \backslash\{\infty\}$. Then $T_{0}$ is also a cover starter.

Proof. First note that the fixed point must occur at least once in $T$ since $T$ 
contains representatives of type II orbitals.

$T$ contains representative pairs of all the type III and IV orbitals at each distance $s$-apart for $s=1,2, \ldots,(\kappa-1)$ by $T$. Both elements of the pairs from these orbitals are from $X \backslash\{\infty\} . T_{0}$ inherits all the elements in $T$ from $X \backslash\{\infty\}$ in the same positions as $T$. Thus $T_{0}$ contains a representative pair of each orbital at every distance $s$-apart, $s=1,2, \ldots,(\kappa-1)$. Now, by construction of $T_{0}$ it is a $k \times 1$ array with only one instance of $\infty$ which gives that the type II orbitals at every distance $s$-apart, $s=1,2, \ldots,(\kappa-1)$ are contained in $T$. Thus $T_{0}$ is a valid cover starter.

The arrays generated by both styles of single fixed point construction generate arrays that are of size $(v-1) \times k+1$ and thus produce covering arrays with parameters $C A((v-1) k+1 ; v, k)$.

\subsubsection{Entire- $k$ Style Construction}

The Entire- $k$ style requires a cover starter of length $k$. The array $M$ is the circulant array produced from the cover starter and is shown in Table 4.4. The cover starters cover all the type II, III, and IV orbitals at all the distances apart in the cover starter. By the enumerations given in Section 3.3 there are $2(v-|G|)$ Type II orbitals, 1 Type II, and $(|G|-1)$ Type IV orbitals giving $v+1$ of these three types of orbitals. Since $k$ must cover all these orbitals at each distance $k \geq v+1$. Table 4.9 is a $C A(29 ; 7,5)$ that is formed by this construction with the group $\mathbb{Z}_{4}$.

Theorem 4.5. [Entire-k Single Fixed Point Construction] Let $X$ be a set and $G$ be a group where $X=\left\{\infty, g_{1}, g_{2}, \ldots, g_{|G|}\right\}$ and $G$ be a group that 
Table 4.9: A $C A(29 ; 7,5)$ by the Meagher Stevens single fixed point construction

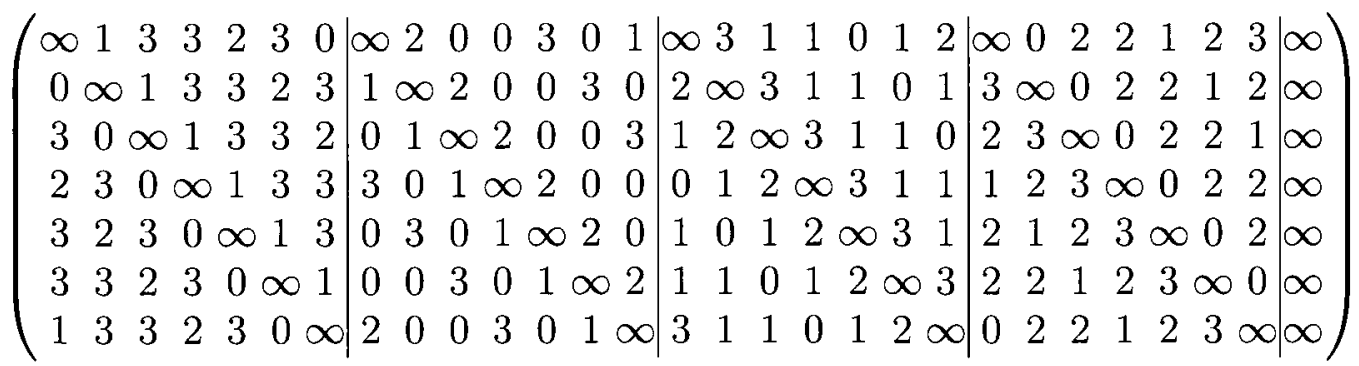

acts regularly on $X \backslash\{\infty\}$. As well, let $T$ be a cover starter of size $k \times 1$ that contains a representative pair of all type II, III, and IV orbitals at each distance $s$-apart, $s=1,2, \ldots,(k-1)$. If $M$ is the circulant array formed from the starter and $C$ is a $k \times 1$ array with $\infty$ in every position, then $\left[M^{G}, C\right]$ is a covering array, $C A(k(v-1)+1 ; k, v)$.

Proof. Since the Entire- $k$ single point cover starter contains a representative pair of all the Type II, III, and IV orbitals Lemma 3.4 and the Orbit Covering Lemma 3.1 give that $M^{G}$ is covers all the pairs of the Type II, III, and IV orbitals.

$C$ by definition covers all the Type I pairs.

\subsubsection{Partial- $k$ Style Construction}

Colbourn et al developed this variant of the single fixed point construction [10]. The cover starter is of length $k-1$ and contains all the type II and type IV orbitals at $s$-apart distances for $s=1,2, \ldots,(k-2)$. Table 4.10 
gives the array $M$ of this construction. The lower limit on $k$ is enforced by both the number of orbitals that the cover starter needs to cover, which is the sum of the Type II, III, and IV orbitals is $v=2+(|G|-1)=v-1$, and the number of elements, $v$, that are needed in the cover starter. This gives that $k-1 \geq v$, which is the same naive bound on $k$ as for the Entire- $k$ single fixed point construction. Table 4.11 is a $C A(36 ; 7,5)$ developed with this construction where the group used is $\mathbb{Z}_{4}$.

Table 4.10: The Array $M$ Partial- $k$ single fixed point construction.

$$
\left(\begin{array}{ccccc}
x_{1} & x_{k-1} & \ldots & x_{2} & g_{1} \\
x_{2} & x_{1} & \ldots & x_{3} & g_{1} \\
x_{3} & x_{2} & \ldots & x_{4} & g_{1} \\
\vdots & \vdots & \ddots & \vdots & g_{1} \\
x_{k-1} & x_{k-2} & \ldots & x_{1} & g_{1} \\
g_{1} & g_{1} & g_{1} & g_{1} & \infty
\end{array}\right)
$$

Table 4.11: A $C A(36 ; 7,5)$ by the Partial- $k$ Single Fixed Point Construction.

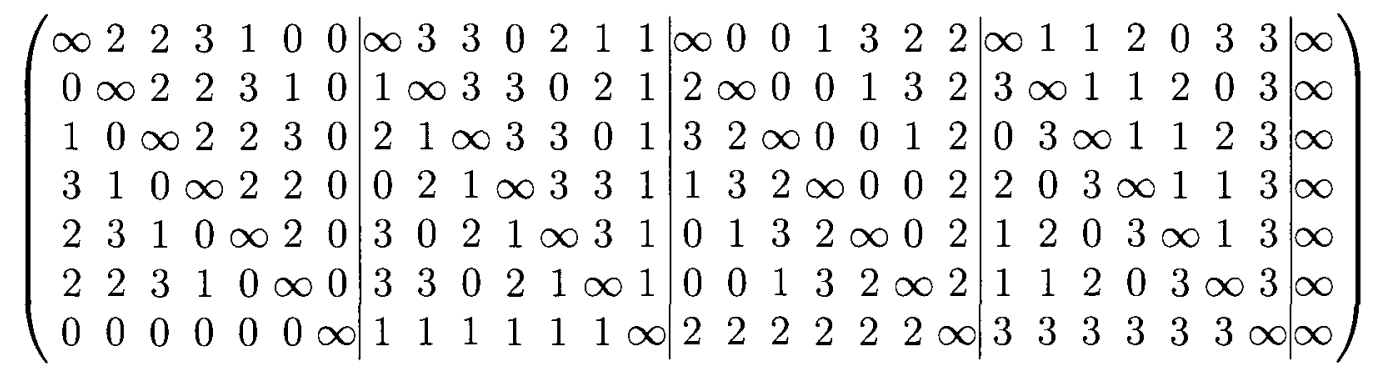


Theorem 4.6. [Partial-k Single Fixed Point Construction] Let $X$ be a set and $G$ be a group where $X=\left\{\infty, g_{1}, g_{2}, \ldots, g_{|G|}\right\}$ and $G$ act regularly on $X \backslash\{\infty\}$. Let $T$ be a cover starter of size $(k-1) \times 1$ which covers all type II and IV orbitals at each distance $s$-apart, $s=1,2, \ldots,(k-2)$. Let $C$ be $a k \times 1$ array with $\infty$ in every position. Form an array, $M$, from Partial- $k$ single fixed point $T$. Then the $\left[M^{G}, C\right]$ construction yields a covering array

Proof. The type II, III, and IV orbitals are covered via Theorem 3.1 and $M$. These orbitals are covered in four cases.

Case 1: Since the Partial- $k$ no fixed point cover starter contains a representative pair of every Type II and IV orbital, Lemma 3.4 give there is a representative pair for every Type II and IV orbit in each pair of rows $i, j$ for all $i, j=1, \ldots, k-1$ and $i \neq j$.

Case 2: Let $(x, y)$ be a pair from a type III orbital with elements from distinct rows $i, j$ in $M$ where $i, j=1, \ldots,(k-1)$. The final column in $M$ gives a representative pair by construction.

Case 3: Let $(x, y)$ be a pair from a type III or IV orbital with one element from row $k$ in $M$ and the other in a row $1, \ldots,(k-1)$. By construction $g_{1}$ is placed in row $k$ of $M$ from the first $k-1$ columns and $T$ has every element from $\left\{g_{1}, g_{2}, \ldots, g_{|G|}\right\}$ in some position. The circulant array of $T$ is a subarray that comprises the first $(k-1)$ rows and columns of $M$. Thus there exists a column, $j$, of $M$ where $g_{1}$ is in row $k$ with $g_{i} \forall\left\{g_{1}, \ldots, g_{|G|}\right\}$ in a given row of the first $(k-1)$ rows. Consider the pair $\left(g_{1}, g_{\alpha}\right)$ and $\left(g_{1}, g_{\beta}\right), \alpha \neq \beta$ cannot be in the same orbital. Thus by the pigeon hole principle all the orbitals of type III and IV have a representative at every distance apart when one element of the pair is in row $k$. 
Case 4: Let $(x, y)$ be a pair from a type II orbital with one element from row $k$. For the pairs that have $\infty$ in row $k$ then it is easy to see that by construction column $k$ of $M$ yields a representative pair for all possible positions. By construction $T$ contains $\infty$ and thus $\infty$ is contained in some row of the circulant of $T$. Thus in $M$ columns $\infty$ occurs in all the rows $1, \ldots,(k-1)$ somewhere in the first $k-1$ columns. Also, in the first $(k-1)$

columns $g_{1}$ is in row $k$. This gives a representative pair for distinct rows $i, j$ for $i, j=1, \ldots, k$ of the type II orbitals

The four cases give a representative of every orbital of type II, III, and IV in some column of $M$ for any two distinct rows of $M$. Thus by the orbital covering lemma $M^{G}$ is a covering array.

By construction $C$ covers the type I orbitals. Thus $\left[M^{G}, C\right]$ is a covering array.

\subsection{Double Fixed Point}

\subsubsection{Introduction and the Simple Construction}

The double fixed point constructions have $X=\left\{\infty_{1}, \infty_{2}, g_{1}, g_{2}, \ldots, g_{|G|}\right\}$ the action of $G$ is regular on $X \backslash\left\{\infty_{1}, \infty_{2}\right\}$ and fixes $\left\{\infty_{1}, \infty_{2}\right\}$. An example of the orbitals of such a group action where the group is $\mathbb{Z}_{4}$ is shown in Table 4.12. In this case the number of orbitals of the differing types are 4 Type I, 4 Type II, 1 Type III, and $|G|-1$ Type IV. In all cases the array $C$ covers all pairs of the type I orbitals. To accomplish this $C$ is a $C A(N ; 2, k)$ where the set of elements is $\left\{\infty_{1}, \infty_{2}\right\}$ and $N=C A N(k, v)$. The covering array numbers of $C A(N ; 2, k)$ were given by Katona [14]. $C A N(2, k)$ is noted as $\mathcal{N}_{k}$ in this 
thesis. $M^{G}$ covers all the pairs from the type II, II and IV orbitals.

Table 4.12: Orbitals Resulting From a the Group $\mathbb{Z}_{4}$ Acting Regularly on $X \backslash\left\{\infty_{1}, \infty_{2}\right\}$ and Fixes $\left\{\infty_{1}, \infty_{2}\right\}$.

$$
\begin{aligned}
& B_{1}=\left\{\left(\infty_{1}, \infty_{1}\right)\right\} \\
& B_{2}=\left\{\left(\infty_{1}, \infty_{2}\right)\right\} \\
& B_{3}=\left\{\left(\infty_{2}, \infty_{1}\right)\right\} \\
& B_{4}=\left\{\left(\infty_{2}, \infty_{2}\right)\right\} \\
& B_{5}=\left\{\left(\infty_{1}, 0\right),\left(\infty_{1}, 1\right),\left(\infty_{1}, 2\right),\left(\infty_{1}, 3\right)\right\} \\
& B_{6}=\left\{\left(\infty_{2}, 0\right),\left(\infty_{2}, 1\right),\left(\infty_{2}, 2\right),\left(\infty_{2}, 3\right)\right\} \\
& B_{7}=\left\{\left(0, \infty_{1}\right),\left(1, \infty_{1}\right),\left(2, \infty_{1}\right),\left(3, \infty_{1}\right)\right\} \\
& B_{8}=\left\{\left(0, \infty_{2}\right),\left(1, \infty_{2}\right),\left(2, \infty_{2}\right),\left(3, \infty_{2}\right)\right\} \\
& B_{9}=\{(0,0),(1,1),(2,2),(3,3)\} \\
& B_{10}=\{(0,1),(1,2),(2,3),(3,0)\} \\
& B_{11}=\{(0,2),(1,3),(2,0),(3,1)\} \\
& B_{12}=\{(0,3),(1,0),(2,1),(3,2)\}
\end{aligned}
$$

There is a complication that arises from having multiple fixed points in a cover starter. In the other constructions the circulant of the cover starter is solely responsible for covering type II orbits over a range of rows. When there is only one instance of each of the two fixed points, $\left\{\infty_{1}, \infty_{2}\right\}$, in the cover starter the cover starter can not contain all the Type II orbits. In this case $\infty_{1}$ is at some distance $d$-apart from $\infty_{2}$. This is the only occurrence of $\infty_{1}$ and thus cannot be $d$-apart from some $g_{i} \in X$. Hence there is no representative pair at this distance apart from the Type II orbitals of the 
form $\left(\infty_{1}, g_{i}\right)$ and $\left(g_{i}, \infty_{2}\right)$ at distance $d$ when there is only one instance of each fixed point in the starter. This also applies to the type II orbitals $\left(g_{i}, \infty_{1}\right)$ and $\left(\infty_{2}, g_{i}\right)$ at distance $-d \bmod (m-1)$ but if one of these sets of pairs is contained at their respective distance apart then the counterpart is found at the other distance apart. If the circulant is solely responsible for the type II orbits then more than one instance of each of the fixed points must occur in the cover starter. Thus two different types of $M$ are used in constructions of each the Entire- $k$ and Partial- $k$ style.

The two types of $M$ have disparate approaches to finding new least upper bounds for covering arrays. As discussed in Section 4.4.4 and 4.4.5. The one approach is a the Simple double fixed point construction whose $M$ 's are similar to the other constructions and the circulant covers the all the Type II orbitals. The Augmented constructions require additional columns to cover the exceptional Type II orbitals at distance $d$ apart. For this simple $M$, in both styles of construction, the lower bound on $k$ is $v+4$. The other construction, the Augmented double fixed point, the has a bound on $k$ of $v+2$. The Simple fixed point construction has a high lower bound on $k$ relative to $v$ and the Augmented are less efficient but can be found for much lower values of $k$ more detail of this comparison is given in Section 5.2.

\subsubsection{The Augmented Constructions}

The Augmented double fixed point has extra columns in its $M$ that contain representatives of the orbitals that can not be covered at distance $d$-apart when there is a single occurrence of each fixed point in the cover starter. The advantage over the Simple double fixed point type of $M$ is that the 
lower bound on $k$ is $v+2$. The hope is that these less efficient constructions are able to be found for small enough $k$ to yield new bounds despite the inefficiencies of construction.

A $\kappa \times \phi$ array, $E$, is now introduced. The purpose of $E$ is to contain the Type II orbits $\left(\infty_{1}, g_{i}\right)$ and $\left(g_{i}, \infty_{2}\right)$ at distance $d$-apart when $\left(\infty_{1}, \infty_{2}\right)$ is at distance $d$-apart in a cover starter. $E$ is a subarray of $M$ that is concatenated horizontally with the $\kappa \times \kappa$ circulant array formed from the cover starter. See Table 4.13 for a generalized example. The array $E$ has $\phi=3$ or 4 . If $3 d \mid \kappa$ then $\phi=3$, otherwise $\phi=4$. The entries of $E$ are largely made of $d$ sized blocks of $\infty_{1}, \infty_{2}$, and an arbitrarily chosen $g_{i}$. Here $g_{1}$ is chosen. Table 4.18 gives examples of the four possibilities of $E$. If $3 d \mid \kappa$ then the $E$ defined by Table 4.14 is used because $M$ has one less column than the other possibilities of $E$. If $3 d \nmid \kappa$ then the $E$ defined in Tables $4.15,4.16$, and 4.17 are used when $2 d \mid \kappa, \kappa \equiv 1, \ldots, d \bmod 2 d$ and $\kappa \equiv(d+1), \ldots,(2 d-1)$ $\bmod 2 d$ respectively. The proof of the coverage properties of $E$ is given by Theorem 4.7.

Table 4.13: The Concatenation of a Circulant Array with an the Array E.

$$
\left(\begin{array}{cccc}
x_{1} & x_{\kappa} & \ldots & x_{2} \\
x_{2} & x_{1} & \ldots & x_{3} \\
x_{3} & x_{2} & \ldots & x_{4} \\
\vdots & \vdots & \ddots & \vdots \\
x_{k} & x_{\kappa-1} & \ldots & x_{1}
\end{array}\right)
$$


Table 4.14: Definition of subarray $E$ of $M$ used when $3 d \mid \kappa$.

$$
e_{i j}=\left\{\begin{array}{llll}
\infty_{1} & j=1 & \left\lceil\frac{i}{3 d}\right\rceil=0 & \bmod 3 \\
& j=2 & \left\lceil\frac{i}{3 d}\right\rceil=1 & \bmod 3 \\
& j=3 & \left\lceil\frac{i}{3 d}\right\rceil=2 & \bmod 3 \\
g_{1} & j=1 & \left\lceil\frac{i}{3 d}\right\rceil=2 & \bmod 3 \\
j=2 & \left\lceil\frac{i}{3 d}\right\rceil=0 & \bmod 3 \\
j=3 & \left\lceil\frac{i}{3 d}\right\rceil=1 & \bmod 3 \\
\infty_{2} & j=1 & \left\lceil\frac{i}{3 d}\right\rceil=1 & \bmod 3 \\
j=2 & \left\lceil\frac{i}{3 d}\right\rceil=2 & \bmod 3 \\
j=3 & \left\lceil\frac{i}{3 d}\right\rceil=0 & \bmod 3
\end{array}\right.
$$

Table 4.15: Definition of subarray $E$ of $M$ when $2 d \mid m$ and $3 d \nmid \kappa$.

$$
e_{i j}=\left\{\begin{array}{rrrr}
\infty_{1} & j=1 & \left\lceil\frac{i}{2 d}\right\rceil=0 & \bmod 2 \\
& j=2 & \left\lceil\frac{i}{2 d}\right\rceil=1 & \bmod 2 \\
g_{1} & j=1 & \left\lceil\frac{i}{2 d}\right\rceil=1 & \bmod 2 \\
j=2 & \left\lceil\frac{i}{2 d}\right\rceil=0 & \bmod 2 \\
j=3 & \left\lceil\frac{i}{2 d}\right\rceil=1 & \bmod 2 \\
j=4 & \left\lceil\frac{i}{2 d}\right\rceil=0 & \bmod 2 \\
\infty_{2} & j=3 & \left\lceil\frac{i}{2 d}\right\rceil=0 & \bmod 2 \\
& j=4 & \left\lceil\frac{i}{2 d}\right\rceil=1 & \bmod 2
\end{array}\right.
$$


Table 4.16: Definition of the subarray $E$ of $M$ when $\kappa \equiv 1, \ldots, d \bmod 2 d$ and $3 d \nmid \kappa$.

$$
e_{i j}=\left\{\begin{array}{rll}
\infty_{1} & j=1 & \left\lceil\frac{i}{2 d}\right\rceil=0 \bmod 2 \text { for } i \leq m-r_{m} \\
j=2 & \left\lceil\frac{i}{2 d}\right\rceil=1 \bmod 2 \text { for } i \leq m-r_{m} \\
j=4 & i=\left(m-r_{m}\right), \ldots, m \\
g_{1} & j=1 & \left\lceil\frac{i}{2 d}\right\rceil=1 \bmod 2 \text { for } i \leq m-r_{m} \text { and } i=\left(m-r_{m}\right), \ldots, m \\
j=2 & \left\lceil\frac{i}{2 d}\right\rceil=0 \bmod 2 \text { for } i \leq m-r_{m} \\
j=3 & \left\lceil\frac{i}{2 d}\right\rceil=1 \bmod 2 \text { for } i \leq m-r_{m} \text { and } i=\left(m-r_{m}\right), \ldots, m \\
j=4 & \left\lceil\frac{i}{2 d}\right\rceil=0 \bmod 2 \text { for } i \leq m-r_{m} \\
\infty_{2} j=1 \quad i=\left(m-r_{n}\right), \ldots, m \\
j=3 & \left\lceil\frac{i}{2 d}\right\rceil=0 \bmod 2 \text { for } i \leq m-r_{m} \\
j=4 & \left\lceil\frac{i}{2 d}\right\rceil=1 \bmod 2 \text { for } i \leq m-r_{m}
\end{array}\right.
$$

Table 4.17: Definition of the $E$ when $\kappa \equiv(d+1), \ldots,(2 d-1) \bmod 2 d$ and

$$
\begin{aligned}
& 3 d \nmid \kappa \text {. } \\
& e_{i j}=\left\{\begin{array}{rll}
\infty_{1} \quad j=1 & \left\lceil\frac{i}{d}\right\rceil=0 \bmod 2 \text { for } i \leq m-d \\
j=2 & \left\lceil\frac{i}{2 d}\right\rceil=1 \bmod 2 \text { for } i \leq m-d \text { and } i=(m-d+1), \ldots,\left(m-r_{m}\right) \\
j=4 & i=(m-d+1), \ldots,\left(m-r_{m}\right) \\
g_{1} \quad j=1 & \left\lceil\frac{i}{2 d}\right\rceil=1 \bmod 2 \text { for } i \leq m-d \text { and } i=\left(m-r_{m}+1\right), \ldots, m \\
j=2 & \left\lceil\frac{i}{2 d}\right\rceil=0 \bmod 2 \text { for } i \leq m-d \text { and } i=(m-d+1), \ldots\left(m-r_{m}\right) \\
j=3 & \left\lceil\frac{i}{2 d}\right\rceil=1 \bmod 2 \text { for } i \leq m-d \text { and } i=(m-d+1), \ldots, m \\
j=4 & \left\lceil\frac{i}{2 d}\right\rceil=0 \bmod 2 \text { for } i \leq m-d \\
\infty_{2} \quad j=1 & i=(m-d+1), \ldots,\left(m-r_{m}\right) \\
j=3 & \left\lceil\frac{i}{2 d}\right\rceil=0 \bmod 2 \text { for } i \leq m-d \\
j=4 & \left\lceil\frac{i}{2 d}\right\rceil=1 \bmod 2 \text { for } i \leq m-d \text { and } i=\left(m-r_{m}+1\right), \ldots, m
\end{array}\right.
\end{aligned}
$$


Table 4.18: Examples of $\mathrm{E}$ for $3 d|\kappa, 2 d| \kappa, 2 d \equiv 0, \ldots, d \bmod 2 d$, and $\kappa \equiv(d+1), \ldots,(2 d-1) \bmod 2 d$ respectively.

\begin{tabular}{|c|c|c|c|}
\hline$\infty_{1} \infty_{2} g_{1}$ & $\infty_{1} \quad g_{1} \quad \infty_{2} \quad g_{1}$ & $\infty_{1} \quad g_{1} \infty_{2} \quad g_{1}$ & $\infty_{1} \quad g_{1} \quad \infty_{2} g_{1}$ \\
\hline $\mathrm{o}_{1} \infty_{2} g_{1}$ & $\begin{array}{llll}\infty_{1} & g_{1} & \infty_{2} & g_{1}\end{array}$ & $\infty_{1} \quad g_{1} \quad \infty_{2} \quad g_{1}$ & $\infty_{1} \quad g_{1} \infty_{2} \quad g_{1}$ \\
\hline${ }_{1} \infty_{2} \quad g_{1}$ & $\infty_{1} \quad g_{1} \quad \infty_{2} \quad g_{1}$ & $\infty_{1} \quad g_{1} \quad \infty_{2} \quad g_{1}$ & $\infty_{1} g_{1} \infty_{2} g_{1}$ \\
\hline$g_{1} \infty_{1} \infty_{2}$ & $\begin{array}{llll}g_{1} & \infty_{1} & g_{1} & \infty_{2}\end{array}$ & $\begin{array}{llll}g_{1} & \infty_{1} & g_{1} & \infty_{2}\end{array}$ & $\begin{array}{llll}g_{1} & \infty_{1} & g_{1} & \infty_{2}\end{array}$ \\
\hline${ }_{1} \infty_{1} \infty_{2}$ & $\begin{array}{llll}g_{1} & \infty_{1} & g_{1} & \infty_{2}\end{array}$ & $\begin{array}{llll}g_{1} & \infty_{1} & g_{1} & \infty_{2}\end{array}$ & $\begin{array}{llll}g_{1} & \infty_{1} & g_{1} & \infty\end{array}$ \\
\hline$g_{1} \infty_{1} \infty_{2}$ & $\begin{array}{llll}g_{1} & \infty_{1} & g_{1} & \infty_{2}\end{array}$ & $\begin{array}{llll}g_{1} & \infty_{1} & g_{1} & \infty_{2}\end{array}$ & $\begin{array}{llll}g_{1} & \infty_{1} & g_{1} & \infty_{2}\end{array}$ \\
\hline$\infty_{2} \quad g_{1} \infty_{1}$ & $\begin{array}{llll}\infty_{1} & g_{1} & \infty_{2} & g_{1}\end{array}$ & $\infty_{1} \quad g_{1} \quad \infty_{2} \quad g_{1}$ & $\infty_{1} \quad g_{1} \quad \infty_{2} \quad g_{1}$ \\
\hline$\infty_{2} g_{1} \infty_{1}$ & $\infty_{1} \quad g_{1} \infty_{2} g_{1}$ & $\infty_{1} \quad g_{1} \infty_{2} \quad g_{1}$ & $\infty_{1} g_{1} \quad \infty_{2} \quad g_{1}$ \\
\hline$\infty_{2} \quad g_{1} \infty_{1}$ & $\infty_{1} \quad g_{1} \quad \infty_{2} \quad g_{1}$ & $\infty_{1} \quad g_{1} \infty_{2} \quad g_{1}$ & $\infty_{1} \quad g_{1} \infty_{2} \quad g_{1}$ \\
\hline${ }_{1} \infty_{2} g_{1}$ & $\begin{array}{llll}g_{1} & \infty_{1} & g_{1} & \infty_{2}\end{array}$ & $\begin{array}{llll}g_{1} & \infty_{1} & g_{1} & \infty_{2}\end{array}$ & $\begin{array}{lll}g_{1} & \infty_{1} \quad g_{1} \quad \infty_{2}\end{array}$ \\
\hline$\infty_{1} \infty_{2} \quad g_{1}$ & $\begin{array}{llll}g_{1} & \infty_{1} & g_{1} & \infty_{2}\end{array}$ & $g_{1} \infty_{1} g_{1} \infty_{2}$ & $\begin{array}{llll}g_{1} & \infty_{1} & g_{1} & \infty_{2}\end{array}$ \\
\hline$\infty_{1} \infty_{2} g_{1}$ & $\begin{array}{llll}g_{1} & \infty_{1} & g_{1} & \infty_{2}\end{array}$ & $\begin{array}{llll}g_{1} & \infty_{1} & g_{1} & \infty_{2}\end{array}$ & $\begin{array}{llll}g_{1} & \infty_{1} & g_{1} & \infty_{2}\end{array}$ \\
\hline$g_{1} \infty_{1} \infty_{2}$ & $\infty_{1} g_{1} \infty_{2} g_{1}$ & $\infty_{2} \quad g_{1} \quad g_{1} \quad \infty_{1}$ & $\infty_{1} g_{1} \infty_{2} g_{1}$ \\
\hline$g_{1} \infty_{1} \infty_{2}$ & $\infty_{1} \quad g_{1} \infty_{2} g_{1}$ & $\begin{array}{llll}\infty_{2} & g_{1} & g_{1} & \infty_{1}\end{array}$ & $\infty_{2} \quad g_{1} \quad g_{1} \quad \infty_{1}$ \\
\hline$g_{1} \infty_{1} \infty_{2}$ & $\infty_{1} g_{1} \infty_{2} g$ & & $\infty_{2} \quad g_{1} \quad g_{1} \quad \infty_{1}$ \\
\hline$\infty_{2} \quad g_{1}$ & $\begin{array}{llll}g_{1} & \infty_{1} & g_{1} & \circ\end{array}$ & & $\begin{array}{llll}g_{1} & \infty_{1} & g_{1} & \infty_{2}\end{array}$ \\
\hline $2 g_{1} \propto$ & $\begin{array}{llll}g_{1} & \infty_{1} & g_{1} & \circ\end{array}$ & & \\
\hline $\mathrm{O}_{2} \quad g_{1}$ & $g_{1}$ & & \\
\hline
\end{tabular}


Theorem 4.7. Each $E$ defined in Tables 4.14, 4.15, 4.16, and 4.17 contain the pairs $\left(\infty_{1}, g_{1}\right)$ and $\left(g_{1}, \infty_{2}\right)$ in all rows $i, j j-i \equiv d \bmod \kappa$ and $i \neq j$.

Proof. Note if $\infty_{2}$ is at distance $-d \bmod \kappa$-apart from $g_{1}$ then $g_{1}$ is at distance $d$-apart from $\infty_{2}$

Case $1(3 d \mid \kappa)$ : By definition the following entries $E$ of $\infty_{1}$ are at distance $d$-apart from $g_{1}$ :

$$
\begin{aligned}
& j=1\left\lceil\frac{i}{3 d}\right\rceil=0 \bmod 3 \\
& j=2\left\lceil\frac{i}{3 d}\right\rceil=1 \bmod 3 \\
& j=3\left\lceil\frac{i}{3 d}\right\rceil=2 \bmod 3
\end{aligned}
$$

By definition the following entries $E$ of $\infty_{2}$ are at distance $-d \bmod \kappa$ apart from $g_{1}$ :

$$
\begin{aligned}
& j=1\left\lceil\frac{i}{3 d}\right\rceil=1 \bmod 3 \\
& j=2\left\lceil\frac{i}{3 d}\right\rceil=2 \bmod 3 \\
& j=3\left\lceil\frac{i}{3 d}\right\rceil=0 \bmod 3
\end{aligned}
$$

Case $2(2 d \mid \kappa)$ : By definition the following entries $E$ of $\infty_{1}$ are at distance $d$-apart from $g_{1}$ :

$$
\begin{aligned}
& j=1\left\lceil\frac{i}{2 d}\right\rceil=0 \bmod 2 \\
& j=2\left\lceil\frac{i}{2 d}\right\rceil=1 \bmod 2
\end{aligned}
$$

By definition the following entries $E$ of $\infty_{2}$ are at distance $-d \bmod \kappa$ apart from $g_{1}$ :

$$
\begin{aligned}
& j=3\left\lceil\frac{i}{2 d}\right\rceil=0 \bmod 2 \\
& j=4\left\lceil\frac{i}{2 d}\right\rceil=1 \bmod 2
\end{aligned}
$$


Case 3: $(\kappa \equiv 1, \ldots, d \bmod 2 d)$ : By definition the following entries $E$ of $\infty_{1}$ are at distance $d$-apart from $g_{1}$ :

$$
\begin{aligned}
& j=1\left\lceil\frac{i}{2 d}\right\rceil=0 \bmod 2 \text { for } i \leq \kappa-d \\
& j=2\left\lceil\frac{i}{2 d}\right\rceil=1 \bmod 2 \text { for } i \leq \kappa-d \text { and } i=(\kappa-d+1), \ldots,\left(\kappa-r_{\kappa}\right) \\
& j=4 i=\left(\kappa-d+1, \ldots,\left(\kappa-r_{\kappa}\right)\right.
\end{aligned}
$$

By definition the following entries $E$ of $\infty_{2}$ are at distance $-d \bmod \kappa$-apart from $g_{1}$ :

$$
\begin{aligned}
& j=1 i=\left(\kappa-r_{n}\right), \ldots, \kappa \\
& j=3\left\lceil\frac{i}{2 d}\right\rceil=0 \bmod 2 \text { for } i \leq \kappa-r_{\kappa} \\
& j=4\left\lceil\frac{i}{2 d}\right\rceil=1 \bmod 2 \text { for } i \leq \kappa-r_{\kappa}
\end{aligned}
$$

Case $4 \kappa \equiv(d+1), \ldots,(2 d-1) \bmod 2 d$ By definition the following entries $E$ of $\infty_{1}$ are at distance $d$-apart from $g_{1}$ :

$$
\begin{aligned}
& j=1\left\lceil\frac{i}{2 d}\right\rceil=0 \bmod 2 \text { for } i \leq \kappa-d \\
& j=2\left\lceil\frac{i}{2 d}\right\rceil=1 \bmod 2 \text { for } i \leq \kappa-d \text { and } i=(\kappa-d+1), \ldots,\left(\kappa-r_{\kappa}\right) \\
& j=4 i=(\kappa-d+1), \ldots,\left(\kappa-r_{\kappa}\right)
\end{aligned}
$$

By definition the following entries $E$ of $\infty_{2}$ are at distance $-d \bmod \kappa$-apart from $g_{1}$ :

$$
\begin{aligned}
& j=1 i=(\kappa-d+1), \ldots,\left(\kappa-r_{\kappa}\right) \\
& j=3\left\lceil\frac{i}{2 d}\right\rceil=0 \bmod 2 \text { for } i \leq \kappa-d \\
& j=4\left\lceil\frac{i}{2 d}\right\rceil=1 \bmod 2 \text { for } i \leq \kappa-d \text { and } i=\left(\kappa-r_{\kappa}+1\right), \ldots, \kappa
\end{aligned}
$$

Thus in every case the pairs $\left(\infty_{1}, g_{1}\right)$ and $\left(g_{1}, \infty_{2}\right)$ occur in some column for every pair of rows distance $d$-apart. 


\subsubsection{The Search Reductions}

The exhaustive searches for both the double fixed point methods use a reduction on the naive search space of $v^{\kappa}$ possible $\kappa \times 1$ arrays. In the case of the simple double fixed point cover starter a pair from the orbital $\left(\infty_{1}, g_{i}\right)$ is required to be at distance 1-apart. A cyclic shift is used to place this pair in the first two positions of the cover starter. By Theorem $4.1 g_{i}$ can be set to $g_{1}$. This ability to assume $\infty_{1}$ and $g_{1}$ are in the first two positions of a potential cover starter reduces the number of $\kappa \times 1$ arrays to search to $v^{\kappa-2}$. Due to the necessity of multiple $\infty_{i}$ 's in the simple double fixed point cover starter a reduction on the search alphabet is not applicable as is possible in the single fixed point and the augmented double fixed point searches.

The augmented $M$ has more extensive reductions in the naive search space. First note by Theorem 4.8 that only one occurrence of each of the fixed points in the starter is needed in the search. The single occurrence of $\left(\infty_{1}, \infty_{2}\right)$ need not be searched for every $\kappa-1$ possible distances apart as given by Theorem 4.9. This reduces the number of $\kappa \times 1$ arrays to check from $v^{\kappa}$ to $\left\lceil\frac{\kappa}{2}\right\rceil(v-2)^{\kappa-2}$.

Theorem 4.8. Let $X=\left\{\infty_{1}, \infty_{2}, g_{1}, g_{2}, \ldots, g_{|G|}\right\}$ be a set and $G$ be a group that acts regularly on $X \backslash\left\{\infty_{1}, \infty_{2}\right\}$ and fixes $\left\{\infty_{1}, \infty_{2}\right\}$. Let $T$ be a cover starter that contains a representative pair of all the Type II, III, and IV orbitals at the distances $s$-apart for $s=1, \ldots, \kappa-1$ with two possible exceptions: the Type II orbitals of the form $\left(\infty_{1}, g_{i}\right)$ and $\left(g_{i}, \infty_{2}\right)$ at the distance $d$-apart when there exists the pair $\left(\infty_{1}, \infty_{2}\right)$ at distance $d$-apart. Define $\bar{T}$ by replacing all the fixed points in $T$ with non fixed points except for a pair of entries $\left(\infty_{1}, \infty_{2}\right)$ at distance $d$. Then $\bar{T}$ is still a cover starter that contains all the 
representative pairs of the Type II, III, and IV orbits with the exception of the orbitals of the from $\left(\infty_{1}, g_{i}\right)$ and $\left(g_{i}, \infty_{2}\right)$ at distance $d$ apart.

Proof. By assumption $T$ contains representative pairs of all the Type II, III and IV orbitals at each distance $s$-apart for $s=1,2, \ldots,(k-1)$ with the exceptions of the Type II orbitals of the form $\left(\infty_{1}, g_{i}\right)$ and $\left(g_{i}, \infty_{2}\right)$ at the distance $d$-apart. At each distance $s$-apart for $s=1,2, \ldots,(k-1)$ the Type III and IV orbitals are contained by $T$. Both elements of the pairs from these orbitals are from $X \backslash\left\{\infty_{1}, \infty_{2}\right\} . \bar{T}$ inherits all the elements in $T$ from $X \backslash\left\{\infty_{1}, \infty_{2}\right\}$ in the same positions as $T$ and thus $\bar{T}$ contains a representative pair of each Type III and IV orbital at every distance s-apart, $s=1,2, \ldots,(k-1)$. Now, by construction of $\bar{T}$ it is a $\kappa \times 1$ array with only one instance of the pair $\left(\infty_{1}, \infty_{2}\right)$ which gives a representative pair of the Type II orbitals at every distance $s$-apart, $s=1,2, \ldots,(k-1)$ are contained in $\bar{T}$ with the exceptions of $\left(\infty_{1}, g_{i}\right)$ and $\left(g_{i}, \infty_{2}\right)$ at the distance $d$-apart. Thus $T_{0}$ is a valid cover starter.

Theorem 4.9. Let $X=\left\{\infty_{1}, \infty_{2}, g_{1}, g_{2}, \ldots, g_{|G|}\right\}$ be a set and $G$ be a group that acts regularly on $X \backslash\left\{\infty_{1}, \infty_{2}\right\}$ and fixes $\left\{\infty_{1}, \infty_{2}\right\}$. Let $T$ be a cover starter that contains a representative pair of all the Type II, III, and IV orbitals at the distances $s$-apart for $s=1, \ldots, \kappa-1$ with two possible exceptions: the type II orbitals of the form $\left(\infty_{1}, g_{i}\right)$ and $\left(g_{i}, \infty_{2}\right)$ at the distance $d$-apart when there exists the pair $\left(\infty_{1}, \infty_{2}\right)$ at distance $d$-apart. Define $\bar{T}$ by exchanging the $\infty_{1}$ entries with the $\infty_{2}$; then $\bar{T}$ is a valid cover starter.

Proof. By assumption $T$ contains representative pairs of all the type II, III and IV orbitals at each distance $s$-apart for $s=1,2, \ldots,(\kappa-1)$ with the 
exceptions of the type II orbitals of the form $\left(\infty_{1}, g_{i}\right)$ and $\left(g_{i}, \infty_{2}\right)$ at the distance $d$-apart. At each distance $s$-apart for $s=1,2, \ldots,(\kappa-1)$ by $T$ the type III and IV orbitals are contained. $\bar{T}$ inherits all the elements in $T$ from $X \backslash\left\{\infty_{1}, \infty_{2}\right\}$ in the same positions as $T$ and thus $\bar{T}$ contains a representative pair of each orbital at every distance $s$-apart, $s=\{1,2, \ldots,(\kappa-1)\}$.

Now with the $\infty_{i}$ 's replacing each other in the same position gives that the Type II orbitals are covered except for the type II orbitals of the form $\left(\infty_{2}, g_{i}\right)$ and $\left(g_{i}, \infty_{1}\right)$. Thus $\bar{T}$ is a valid Augmented double fixed point cover starter.

\subsubsection{Entire- $k$ Style Constructions}

Both the Simple and Augmented Entire- $k$ style double fixed point constructions use a cover starter of length $k$. The covering arrays that result from the Simple $M$ have $N=(v-2) k+C A N(2, k)$ and for the Augmented $M$ have $N=(v-2)(k+\phi)+C A N(2, k))$. Both types of $M$ cover a representative of all the type II, III, and IV orbitals at the distances $s$-apart for $s=1, \ldots,(k-1)$. In the Simple case the cover starter contains a representative of all the type II, III, and IV orbitals at $1, \ldots,(k-1)$ distances apart. The Augmented case the cover starter contains a representative of all the type II, III, and IV orbitals at $1, \ldots,(k-1)$ distances apart from the possibility of type II orbitals of the form $\left(\infty_{1}, g_{i}\right)$ and $\left(g_{i} . \infty_{2}\right)$ at a distance $d$. The augmented $M$ contains the subarray $E$ which accounts for the orbitals of the form $\left(\infty_{1}, g_{i}\right)$ and $\left(g_{i} . \infty_{2}\right)$ at distance $d$-apart. $E$ is defined by Tables $4.14,4.15,4.16$ and 4.17

In the simple case the array $M$ is the circulant array produced from 
the cover starter and is shown in Table 4.4. The cover starter contains a representative of all the type II, III, and IV orbitals at all the distances $s$ apart $s=1, \ldots,(k-1)$ in the cover starter. There are $(2 \cdot 2)+1+(v-3)=v+2$ of these three types of orbitals. Since $k$ must cover all these orbitals at each distance and there must be multiple occurrences of each $\infty_{i}, k \geq v+4$. The proof that the array $\left[M^{G}, C\right]$ that results from the Entire- $k$ simple double fixed point construction is a $C A(k(v-2) ; k, v)$ is given in Theorem 4.10.

Theorem 4.10. [Entire-k Simple Double Fixed Point Construction] Let $X$ be a set and $G$ be a group where $X=\left\{\infty_{1}, \infty_{2}, g_{1}, g_{2}, \ldots, g_{|G|}\right\}$ and $G$ acts regularly on $X \backslash\left\{\infty_{1}, \infty_{2}\right\}$. Given is a cover starter, $T$, of size $k \times 1$ that has representative pair from all type II, III, and IV orbitals at each distance $s$-apart, $s=1,2, \ldots,(k-1)$. If $M$ is the circulant array formed from the starter and $C$ is an optimal $C A(2, k)$ with entries of $\left\{\infty_{1}, \infty_{2}\right\}$, then $\left[M^{G}, C\right]$ is a covering array, $C A(k(v-2)+C A N(2, k) ; k, v)$.

Proof. Since the Entire- $k$ simple double point cover starter contains a representative pair of all the Type II, III, and IV orbitals Lemma 3.4 and the Orbit Covering Lemma 3.1 give that $M^{G}$ is a covering array covers all the pairs of the Type II, III, and IV orbitals.

In the case of the augmented construction, $M$ is comprised of the circulant array produced from the cover starter with $E$ concatenated. The cover starter contains a representative of all the type II, III, and IV pair orbitals at all the distances apart in the cover starter with the exception of orbitals of the form $\left(\infty_{1}, g_{i}\right)$ and $\left(g_{i}, \infty_{2}\right)$ at the distance $d$ apart. There are $(2 \cdot 2)+1+(v-3)=$ $v+2$ of these three types of orbitals. Since $k$ must cover all these orbitals at 
each distance apart $k \geq 2+v$. This forms the $k \times(k+\phi)$ array, $M$, which is shown in Table 4.19 .

Table 4.19: The $M$ of the Augmented Entire- $k$ Double Fixed Point Construction

$$
\left(\begin{array}{cccc|c}
x_{1} & x_{k} & \ldots & x_{2} \\
x_{2} & x_{1} & \ldots & x_{3} \\
x_{3} & x_{2} & \ldots & x_{4} & \\
\vdots & \vdots & \ddots & \vdots \\
x_{k} & x_{k-1} & \ldots & x_{1}
\end{array}\right)
$$

Theorem 4.11. [Entire- $k$ Augmented Double Fixed Point Construction] Let $X$ be a set and $G$ be a group where $X=\left\{\infty_{1}, \infty_{2}, g_{1}, g_{2}, \ldots, g_{|G|}\right\}$ and $G$ act regularly on $X \backslash\left\{\infty_{1}, \infty_{2}\right\}$ while leaving $\left\{\infty_{1}, \infty_{2}\right\}$ fixed. The cover starter $T$ is of size $k \times 1$ containing a representative pair from all type $I$, $I I I$, and IV orbitals at each distance s-apart, $s=1,2, \ldots,(k-1)$ with two exceptions. The exceptions are the type II orbitals of the form $\left(\infty_{1}, g_{i}\right)$ and $\left(g_{i}, \infty_{2}\right)$ at distance d-apart. Let $M$ be a Entire-k augmented double fixed point construction $M$ and $C$ be an optimal $C A(2, k)$ on $\left(\infty_{1}, \infty_{2}\right)$. Then $\left[M^{G}, C\right]$ is a covering array, $C A((k+\phi)(v-2)+C A N(2, k) ; k, v)$ where $\phi=3$ if $3 d \mid(k-1)$ otherwise $\phi=4$.

Proof. Since the Entire- $k$ augmented double point cover starter contains a representative pair of all the Type II, III, and IV orbitals except the pairs of the Type II orbitals at the distance $d$-apart as noted Lemma 3.4 gives that 
these representative pairs are present in the circulant. Theorem 4.7 ensures that $M$ contains the exceptional Type II representatives needed at distance $d$-apart. Since all orbitals have representative pairs at every distance apart the Orbit Covering Lemma 3.1 gives that $M^{G}$ is a covering array covers all the pairs of the Type II, III, and IV.

By definition of $C$ it covers the Type I pairs.

\subsubsection{Partial- $k$ Style Constructions}

The Partial- $k$ style double fixed point constructions have a cover starter of length $k-1$. As well, in both cases the cover starter must contain all the elements of $X$.

In the simple case the first $k-1$ columns of $M$ are comprised of a ( $k-$ 1) $\times(k-1)$ circulant of the cover starter with a row of $(k-1)$ entries of $g_{1}$ concatenated on the bottom. The last two columns of $M$ columns have $g_{1}$ in the first $(k-1)$ positions and $\infty_{1}$ in the final position of one of the columns and $\infty_{2}$ in the final position of the other. Table 4.20 is a general example of the Simple $M$. The cover starter must account for $(2 \cdot 2)+(v-3)=v+1$ orbitals and have at least two occurrences of each fixed point thus $k \geq v+2$. The covering arrays that result from the Partial- $k$ Simple double fixed construction are of size $(v-2)(k+1)+C A N(2, k))$.

Theorem 4.12. [Simple Partial-k Double Fixed Point Construction] Let $X$ be a set and $G$ be a group where $X=\left\{\infty_{1}, \infty_{2}, g_{1}, g_{2}, \ldots, g_{|G|}\right\}$ and $G$ acts regularly on $X \backslash\left\{\infty_{1}, \infty_{2}\right\}$ and fixes $\left\{\infty_{1}, \infty_{2}\right\}$. Given $T$ is a cover starter of size $(k-1) \times 1$ that covers all type II and IV orbitals at each distance s-apart, $s=1,2, \ldots,(k-2)$. Form a simple Partial-k double fixed point $M$ from 
Table 4.20: The $M$ of the simple Partial- $k$ double fixed point construction

$$
\left(\begin{array}{cccccc}
x_{1} & x_{k-1} & \cdots & x_{2} & g_{1} & g_{1} \\
x_{2} & x_{1} & \cdots & x_{3} & g_{1} & g_{1} \\
x_{3} & x_{2} & \cdots & x_{4} & g_{1} & g_{1} \\
\vdots & \vdots & \ddots & \vdots & \vdots & \vdots \\
x_{k-1} & x_{k-2} & \cdots & x_{1} & g_{1} & g_{1} \\
g_{1} & g_{1} & g_{1} & g_{1} & \infty_{1} & \infty_{2}
\end{array}\right)
$$

the cover starter $T$ and let $C$ be a optimal $C A(2, k)$ on $\left(\infty_{1}, \infty_{2}\right)$. Then the $\left[M^{G}, C\right]$ construction yields a covering array, $C A(k(v-2)+C A N(2, k) ; k, v)$.

Proof. The type II, III, and IV orbitals are covered in $M^{G}$ via Theorem 3.1 These orbitals will be covered in four cases.

Case 1: Since the Partial- $k$ no fixed point cover starter contains a representative pair of every Type II and IV orbital, Lemma 3.4 give there is a representative pair for every Type II and IV orbit in each pair of rows $i, j$ for all $i, j=1, \ldots, k-1$ and $i \neq j$.

Case 2: Let $(x, y)$ be a pair from a type III orbital with elements from rows $1, \ldots,(k-1)$ in $M$. Column $k$ in $M$ gives a representative pair by construction.

Case 3: Let $(x, y)$ be a pair from a type III or IV orbital with one element from row $k$ in $M$. By construction $g_{1}$ is placed in row $k$ of $M$ for the first $k-1$ columns and $T$ has every element from $\left\{g_{1}, g_{2}, \ldots, g_{|G|}\right\}$ in some position. Thus there exists a column $j$ such that the pair $\left(g_{1}, g_{i}\right)$ for $i=2, \ldots,|G|$ at every distance apart. Now the pair $\left(g_{1}, g_{i}\right)$ and $\left(g_{1}, g_{j}\right) i \neq j$ cannot be in 
the same orbital. Thus by the pigeon hole principle all the orbitals of type III and IV have a representative at every distance apart when one element of the pair is in row $k$.

Case 4: Let $(x, y)$ be a pair from a type II orbital with one element from row $k$. For the pairs that have $\infty_{1}$ or $\infty_{2}$ in row $k$ then it is easy to see that by construction column $k$ and $k+1$ of $M$ yields a representative pair for all possible positions. The first $k-1$ columns have $g_{1}$ in row $k$ and since both $\infty_{1}$ and $\infty_{2}$ are in $T$ by construction, somewhere in the first $k-1$ columns there is a representative pair present.

The four cases give a representative of every orbital of type II, III, and IV at each distance apart in some column of $M$. Thus by the orbital covering lemma $M^{G}$ is a covering array.

By construction $C$ covers the type I orbitals. Thus $\left[M^{G}, C\right]$ is a covering array.

The construction with extra columns in $M$ needs the cover starter to contain a representative pair of all the type II and IV orbitals at the distances $s$-apart for $=1, \ldots, k-2$ with two exceptions. The exceptions are the type II orbitals of the form $\left(\infty_{1}, g_{i}\right)\left(g_{i}, \infty_{2}\right)$ at distance apart, $d$. The cover starter must cover $(2 \cdot 2)+(v-3)=v+1$ orbitals for all but one distance apart thus $k \geq v+2$. Theorem 4.12 demonstrates that array $\left[M^{G}, C\right]$ produces the cover starter via the construction is a covering array.

The $M$ of this construction is formed by concatenating four subarrays. The $(k-1) \times(k-1)$ circulant developed from the cover starter is horizontally concatenated with a $(k-1) \times 1$ array with $g_{1}$ as entry. Concatenate again 
with the subarray $E$ to form a $(k-1) \times(k+\phi)$ array. A $1 \times(k+\phi)$ array with $g_{1}$ in the first $(k-1)$ entries, $\infty_{1}$ in its $k^{\text {th }}$ entry and $\infty_{2}$ in the last $\phi$ entries is concatenated to form the $k^{\text {th }}$ row of $M$.. Table 4.21 is an example of this $M . E$ is defined by Table 4.14, 4.15, 4.16, or 4.17 and which table is used depends on $d$ and $k$. The Partial- $k$ augmented double fixed point construction yields a covering array of size $N=(v-2)(k+\phi)+C A N(2, k)$.

Table 4.21: The Form of the Array $M$ for the Augmented Partial- $k$ Double Fixed Point Construction.

$$
\left(\begin{array}{ccccc|ccc}
x_{1} & x_{k} & \cdots & x_{2} & g_{1} & & & \\
x_{2} & x_{1} & \cdots & x_{3} & g_{1} & & & \\
x_{3} & x_{2} & \cdots & x_{4} & g_{1} & & E & \\
\vdots & \vdots & \ddots & \vdots & \vdots & & & \\
x_{k} & x_{k-1} & \cdots & x_{1} & g_{1} & & & \\
g_{1} & g_{1} & \cdots & g_{1} \infty_{1} & \infty_{2} & \cdots & \infty_{2}
\end{array}\right)
$$

Theorem 4.13. [Partial-k Augmented Double Fixed Point Construction] Let $X=\left\{\infty_{1}, \infty_{2}, g_{1}, g_{2}, \ldots, g_{|G|}\right\}$ be a set and $G$ be a group that acts regularly on $X \backslash\left\{\infty_{1}, \infty_{2}\right\}$. Given $T$ is a cover starter of size $(k-1) \times 1$ that covers all type II and IV orbitals at each distance $s$-apart, $s=1,2, \ldots,(k-2)$ with two exceptions. The exceptions are the type II orbitals of the form $\left(\infty_{1}, g_{i}\right)$ and $\left(g_{i}, \infty_{2}\right)$ at a distance $d$-apart where the pair $\left(\infty_{1}, \infty_{2}\right)$ is at the distance $d$-apart in $T$. Form a Partial-k double fixed point $M$ with extra columns from $T$ and let $C$ be an optimal $C A(2, k)$ on $\left\{\infty_{1}, \infty_{2}\right\}$. Then the $\left[M^{G}, C\right]$ construction is a $C A((k+3)(v-2)+C A N(2, k) ; k, v)$ if $3 d \mid(k-1)$ else 
$C A((k+3)(v-2)+C A N(2, k) ; k, v)$.

Proof. The Type II, III, and IV orbitals will be covered in $M^{G}$ via Theorem 3.1 These orbitals will be covered in four cases.

Case 1: Since the Entire- $k$ no fixed point cover starter contains a representative pair of all the Type II, III, and IV orbitals except the pairs of the Type II orbitals at the distance $d$-apart as noted Lemma 3.4 Theorem 4.7 insures that $M$ contains the Type II representatives needed at distance $d$-apart

Case 2: Let $(x, y)$ be a pair from a Type III orbital with elements from rows $1, \ldots,(k-1)$ in $M$. Column $k$ in $M$ gives a representative pair by construction.

Case 3: Let $(x, y)$ be a pair from a Type III or IV orbital with one element from row $k$ in $M$. By construction $g_{1}$ is placed in row $k$ of $M$ for the first $k-1$ columns and $T$ has every element from $\left\{g_{1}, g_{2}, \ldots, g_{|G|}\right\}$ in some position, Thus there exists a column $j$ such that the pair $\left(g_{1}, g_{i}\right)$ for $i=2, \ldots,|G|$ at every distance apart. Now the pair $\left(g_{1}, g_{i}\right)$ and $\left(g_{1}, g_{j}\right) i \neq j$ cannot be in the same orbital. Thus by the pigeon hole principle all the orbitals of Type III and IV have a representative at every distance apart when one element of the pair is in row $k$.

Case 4: Let $(x, y)$ be a pair from a Type II orbital with one element from row $k$. The required representative pairs that have $\infty_{1}$ or $\infty_{2}$ in row $k$ are contained in one of two cases. The case when $\infty_{1}$ is in row $k$ is contained in column $k$. The case when $\infty_{2}$ is in row $k$ is contained in the columns where the subarray $E$ is in the first $k-1$ rows. By construction $g_{1}$ is in some column in every position $1, \ldots,(k-1)$. The first $k-1$ columns have $g_{1}$ is in row $k$ and since both $\infty_{1}$ and $\infty_{2}$ are in $T$ by construction, somewhere in 
the first $k-1$ columns there is a representative pairs of the form $\left(g_{1}, \infty_{i}\right)$ is present.

The four cases give a representative of every orbital of Type II, III, and IV at each distance apart in some column of $M$. Thus by the orbital covering lemma $M^{G}$ is a covering array.

By construction $C$ covers the Type I orbitals. Thus $\left[M^{G}, C\right]$ is a covering array.

\subsection{Description of the searches}

In this section exhaustive and heuristic searches are outlined, the reductions on the exhaustive search space for each construction is outlined and a brief explanation of the method of implementation is given. There are common arrays for both types of searches to store the orbitals, keep track of the orbital coverage, and ensure conditions are met for the Partial- $k$ construction.

A set of arrays are employed used by the searches to keep track of various properties. The group action is used to create an array, $P$, of size $v \times v$. The rows and columns are indexed by elements of the set $X$. For a pair $\left(x_{i}, x_{j}\right)$, row numbers correspond to the first element in the pair and the column numbers correspond to the second element. The entry of $p_{i j}$ is the orbital of the pair $\left(x_{i}, x_{j}\right)$. A $\kappa \times 1$ array, $T$, is the potential cover starter. The array, $A$, is the number of occurrences of the orbitals at $s$-apart in $T$. $A$ has $b$ columns that correspond to the orbitals the cover starter is required to contain and the rows correspond to the number of distances apart in the starter, but not all of them; only the first $\left\lfloor\frac{\kappa}{2}\right\rfloor$ distances apart from a entry of $T$. In Sections 
4.2-4 it is given that a cover starter either covers all orbitals of a given type or none of a given type and as such Theorem 4.14 gives that only the first $\left\lfloor\frac{\kappa}{2}\right\rfloor$ distances apart are necessary. In the Partial- $k$ cases the cover starters are required to contain all or all but one element of the set $X$. A $v \times 1$ array, $F$, is assigned such that the entry in the $i^{\text {th }}$ position of $F$ is the number of occurrences of the $i^{\text {th }}$ element of $X$ occurs in $T$. In the exhaustive searches there are also arrays for the Gray code [15] that enumerates all tuples for checking. The position of change of the grey code is referred to as $t_{\delta}$.

Theorem 4.14. Given a finite set $X=\left\{\infty_{1}, \infty_{2}, \ldots, \infty_{\mu}\right\} \cup\left\{g_{1}, g_{2}, \ldots, g_{|G|}\right\}$ and a group $G$ that acts on $X$ define the action of $G$ on $X$ is such that $G$ acts regularly on the elements $\left\{g_{1}, g_{2}, \ldots, g_{|G|}\right\} \in X$ while leaving the other elements of $X,\left\{\infty_{1}, \infty_{2}, \ldots \infty_{\mu}\right\}$, fixed. Then if an $\kappa \times 1$ array, $T$, that has a representative of every orbital of a type, as defined by Table 4.1, at each distance s-apart $\kappa \in\left\{1 \ldots\left\lfloor\frac{\kappa}{2}\right\rfloor\right\}$ apart, then $T$ also has representatives of all the orbitals of that type at the distances s-apart $s \in\left\{\left\lfloor\frac{\kappa}{2}\right\rfloor+1, \ldots, m-1\right\}$ apart.

Proof. Assume such a $\kappa \times 1$ array, $T$, exists.

Consider an arbitrary orbital, $B$, of a given type and an arbitrary distance $\bar{d}$-apart where $\bar{d} \in\left\{\left\lfloor\frac{\kappa}{2}\right\rfloor+1, \ldots, m-1\right\}$ and a pair $(x, y) \in B$ contained in $T$ at distance $d$-apart. If the pair $(x, y) \in B$ is such that the pair's transpose, $(y, x)$, at distance $\hat{d}$-apart such that $\hat{d} \equiv-d \bmod \kappa$ then $B$ has a representative pair at distance $d$-apart.

There exists $B_{0}$ of the same orbital type such that $(y, x) \in B_{0}$ since every pair of an orbital type have the same number of fixed points. Type I and Type II orbitals have their type defined by the number of fixed points. If 
$(x, y)$ is in the Type III orbital then III $(y, x)$ is contained by definition the orbital Type. A pair $(x, y)$ from a Type IV orbital can not have $(x, y)$ as a Type I or II orbital as there are no fixed points in Type IV orbitals and $x \neq y$ by definitions of Type IV orbitals. Thus there exists some $\left(y^{g}, x^{g}\right) \in B_{0}$ at distance $\hat{d}$-apart in $T$. This implies that $\left(x^{g}, y^{g}\right)$ is at distance $d$-apart in $T$.

For $T$ to be a cover starter the entries of $A$ need to have nonzero entries. All the search methods were designed with the idea of reducing the required amount of updating of $A$ as to reduce run-times.

The exhaustive searches then progress through the search spaces via a Gray code [15]. In the case of a Gray code, there is only one entry of $T, t_{\delta}$, changed at any time. Only the orbitals of the pairs with $t_{\delta}$ and the entries at distances $1, \ldots,\left\lfloor\frac{\kappa}{2}\right\rfloor$ need to be updated. An affected pair has one subtracted from the corresponding entry of $A$. $T$ is then updated by changing the $\delta t$ entry in accordance with the Gray code. Now for each pair at distance a $1, \ldots,\left\lfloor\frac{\kappa}{2}\right\rfloor$ from the updated $t_{\delta}$ one is added the entry of $A$ corresponding to the pair. $A$ is tested after each change for zero entries to check if $T$ is a valid starter. The Partial- $k$ case also requires a check of $F$ for zero entries to make sure that the required elements of $X$ are present in the starter.

Efficiencies that make use of the group action and cyclic shifts of the cover starters within $T$ are employed to reduce search spaces of the exhaustive search. Table 4.22 gives a summary of all sizes of the exhaustive search spaces and the theorems that use the group action to reduce the space are given.

The setup of the heuristics is a bit different. The parsing of the group 


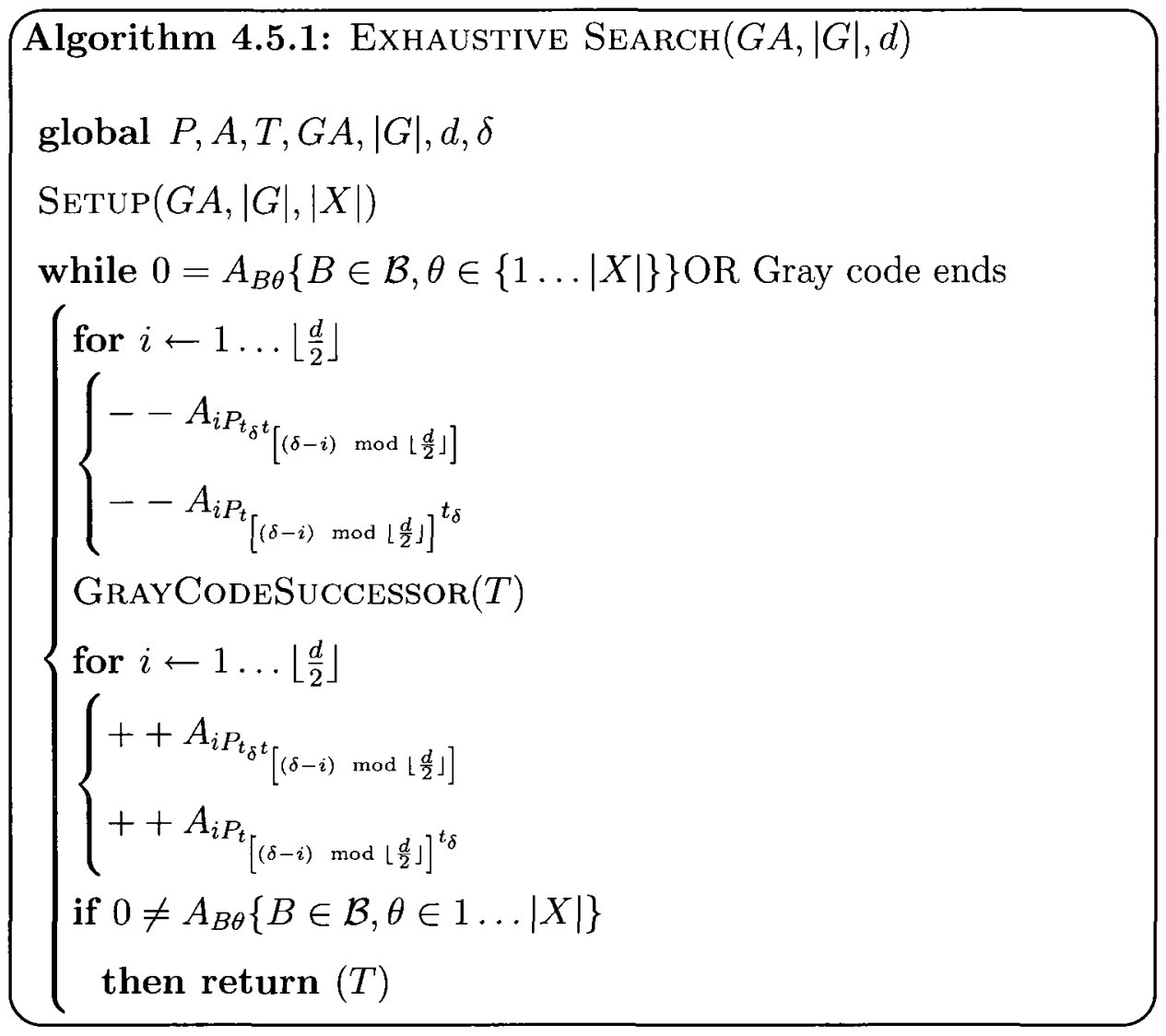

table to $P$ is the only one time setup routine. $T$ has random initial entries from $X$. Then the array $A$ is evaluated according to $T$.

The hillclimb style of heuristic search was used as it is simple to implement and run. Thus, since it succeeded where it was needed, we did not need to examine other meta-heuristics. Even new cover starters are found values of $k$ less then previous searches provided []. In a hill climb the set $\mathcal{T}$ is the set of all the possible $T$ of the search. A cost function, $C(T)$, is defined on all $T \in \mathcal{T}$. A neighbourhood, $N\left(T_{0}\right)$, is defined for all $T_{0} \in \mathcal{T}$ as the set of all 
Table 4.22: Summary of the Search Spaces of the Exhaustive Search

\begin{tabular}{|c|c|c|c|}
\hline Construction & Style & $\begin{array}{c}\text { Size of } \\
\text { Search Space }\end{array}$ & $\begin{array}{l}\text { Theorems } \\
\text { Used }\end{array}$ \\
\hline $\begin{array}{l}\text { No Fixed Points } \\
\text { No Fixed Points }\end{array}$ & $\begin{array}{l}\text { Entire- } k \\
\text { Partial- } k\end{array}$ & $\begin{array}{l}v^{k-1} \\
v^{k-2}\end{array}$ & Theorem 4.1 \\
\hline $\begin{array}{l}\text { Single Fixed Point } \\
\text { Single Fixed Point }\end{array}$ & $\begin{array}{l}\text { Entire- } k \\
\text { Partial- } k\end{array}$ & $\begin{array}{l}(v-1)^{k-2} \\
(v-1)^{k-3}\end{array}$ & $\begin{array}{l}\text { Theorems } 4.1 \\
\text { and } 4.4\end{array}$ \\
\hline $\begin{array}{l}\text { Simple Double Fixed Point } \\
\text { Simple Double Fixed Point }\end{array}$ & $\begin{array}{l}\text { Entire- } k \\
\text { Partial- } k\end{array}$ & $\begin{array}{l}v^{k-2} \\
v^{k-2}\end{array}$ & Theorem 4.1 \\
\hline $\begin{array}{l}\text { Augmented Double Fixed Point } \\
\text { Augmented Double Fixed Point }\end{array}$ & $\begin{array}{l}\text { Entire- } k \\
\text { Partial- } k\end{array}$ & $\begin{array}{c}\left\lceil\frac{k}{2}\right\rceil(v-2)^{k-2} \\
\left\lceil\frac{k-1}{2}\right\rceil(v-2)^{k-3}\end{array}$ & $\begin{array}{l}\text { Theorems } 4.1 \\
4.8, \text { and } 4.9\end{array}$ \\
\hline
\end{tabular}

the $T \in \mathcal{T}$ with a certain degree of similarity to $T_{0}$.

A hill climb is named as such because it follows the 'steepest accent' in its progression. The hill climb first chooses a random initial $T_{0} \in \mathcal{T}$. Then the first iteration of the hill climb is initiated. First $C\left(T_{0}\right)$ is evaluated. Next the $C(T)$ of the $T \in N\left(T_{0}\right)$ are evaluated and a $T^{\prime} \in\left\{\max C(T): T \in N\left(T_{0}\right)\right\}$ is chosen. If $C\left(T^{\prime}\right)>C\left(T_{0}\right)$ then $T^{\prime}$ becomes $T_{0}$ and the another iteration is performed.

The search terminates when no satisfactory $T^{\prime}$ can be found. In some searches, as was done in this thesis, a $T^{\prime}$ such that $C\left(T^{\prime}\right)=C\left(T_{0}\right)$ is allowed. In this case a maximum number of these 'lateral moves', $\sigma$, must be set or the algorithm could get stuck in a loop and not terminate. As well the implementation is such when an individual hill climb search finishes another is started for $T$ with pseudo-random entries. More information on hillclimbs can be found in Kreher and Stinson [16]. 


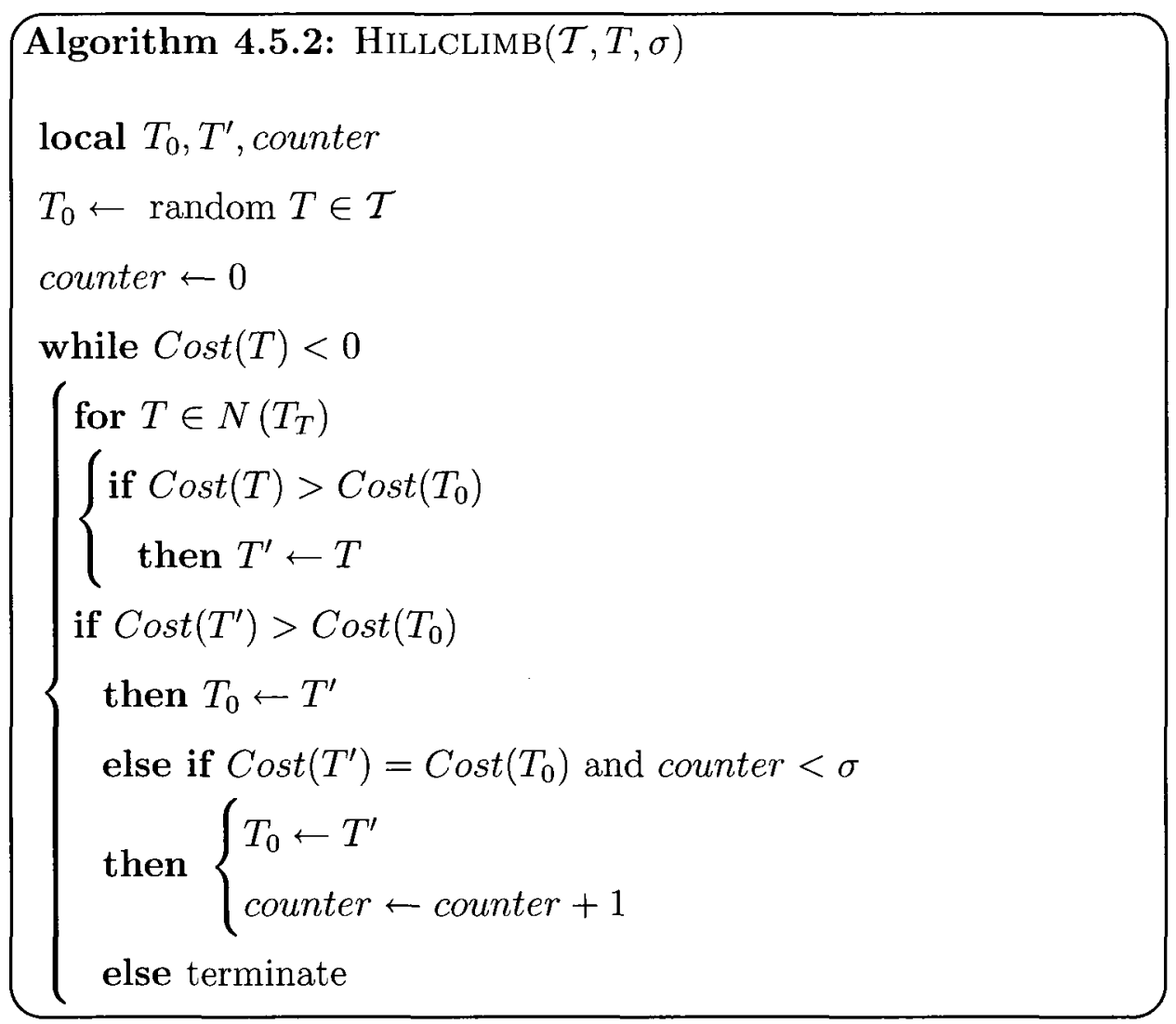

The hill climbs used here has a cost function that is the negative number of zero entries in the array $A$. When $C(T)=0, T$ is a cover starter and there is no need to search further.

The difference in the definition of the neighbourhoods is what sets apart the two hillclimbs used here. The hill climb for the Entire- $k$ is the simpler of the two. The neighbourhood of the hillclimb is all the possible one entry changes to $T$. The Partial- $k$ hill climb is different due to the necessity of a valid $T$ to satisfy the condition of the frequency array $F$. In the Partial- $k$ case the neighbourhood of $T_{0}$ only includes a $T$ if satisfied the frequency condition 
of a construction. This is restrictive on the size of the neighbourhood of a $T_{0}$ and is overcome by allowing all the possible two position changes $T$ such that $F$ has non-zero entries as required.

The exhaustive searches were run for at least a month if not many months in order to find cover starters. For a given $v, G$, and construction exhaustive searching started from lower bound on $k$ and progressed higher. Where the exhaustive searches are infeasible the hill climb would be run from a value of $k$ where with a fair amount of certainty and/or where the construction used would yield a new least upper bound. Then the searches would be initiated for progressively lower values of $k$. 


\section{Chapter 5}

\section{Results and Analysis}

The results of the searches for cover starters given by the eight constructions and analysis of this information is contained in this chapter. The major topics to be addressed are:

- Results and New Least Upper Bounds

- Comparison of Covering Array Sizes by the Different Constructions

- Discontinuities in the Existence Spectra of $k$ for a Given $v$ and $G$ by a Construction

- Expected Existence of a Cover Starter 


\subsection{Results of Searches and New Least Upper Bounds}

The new values for least upper bounds are given in Table 5.5. The values of $k$ for which cover starters exist for a given $v$ and $G$ for each construction are given in Tables 5.1, 5.2, 5.3, and 5.4. The lower bounds on $k$ are listed in italics to the left of the existences. Later in Lemma 5.1 it is shown that the parity of the cover starter length is a determining factor of the lower bound and thus the bounds are given for both odd and even $k$. The upper bounds of cover starter existences are not considered. For one, the constructions do not produce new least upper bounds for $k$ significantly greater than $v$ and Conjecture 5.7 speculates that once a starter is found for each parity then cover starters exist for all higher values of $k$ for a given $v, G$, and construction.

The absolute lower bounds for $v$ of each constructions up to a given value are achieved by exhaustive searches. The absolute lower bounds of no fixed point and single fixed point constructions are found up to $v=10$. Exhaustive searching produces greatest lower bounds on $k$ when $v=11$ for those two types of constructions. Primarily the searches used for the double fixed point are exhaustive. The only exceptions are for the Entire$k$ double fixed point construction for $v=9,10,11$ where a hill climb was used. Exhaustive searching was used until it was unfeasible. At minimum exhaustive runs would be given a month of run time to complete on Intel Xeon $2.8 \mathrm{MHz}$ processors. More information on the implementations of the searches is given in Section 4.5.

Many new least upper bounds on covering arrays are established and 
Table 5.1: Zero Fixed Points Constructions' Greatest Lower Bounds on, and Existences Spectra of $k$

\begin{tabular}{|c|c||c|c||c|c|}
\hline \multirow{2}{*}{$v$} & \multirow{2}{*}{ Group } & \multicolumn{2}{|c||}{ Entire- $k$} & \multicolumn{2}{c|}{ Partial- $k$} \\
\cline { 3 - 6 } & & $\begin{array}{c}\text { Lower Bounds } \\
\text { Odd,Even }\end{array}$ & $\begin{array}{c}\text { Existence } \\
\text { Spectra }\end{array}$ & $\begin{array}{c}\text { Lower Bounds } \\
\text { Odd,Even }\end{array}$ & $\begin{array}{c}\text { Existence } \\
\text { Spectra }\end{array}$ \\
\hline \hline 2 & $\mathbb{Z}_{2}$ & $3_{b}, 4_{e}$ & $3-6$ & $3_{b}, 2_{b}$ & $2-6$ \\
\hline 3 & $\mathbb{Z}_{3}$ & $3_{b}, 4 b$ & $3-8$ & $3_{b}, 4 b$ & $3-8$ \\
\hline 4 & $\mathbb{Z}_{4}$ & $5_{b}, 8_{e}$ & $5,7-10$ & $\gamma_{e}, 6_{e}$ & $6-10$ \\
& $K_{4}$ & $7_{e}, 8_{p}$ & $7-10$ & $\eta_{p}, 4 b$ & $4,6-10$ \\
\hline 5 & $\mathbb{Z}_{5}$ & $5_{b}, 8_{e}$ & $5,7-10$ & $5_{b}, 8_{e}$ & $5,7-10$ \\
\hline 6 & $\mathbb{Z}_{6}$ & $9_{e}, 10_{e}$ & $9-13$ & $9_{e}, 8_{e}$ & $8-13$ \\
& $S_{3}$ & $9_{e}, 10_{p}$ & $9-13$ & $11_{e}, 10_{e}$ & $10-13$ \\
\hline 7 & $\mathbb{Z}_{7}$ & $7_{b}, 10_{e}$ & $7,9-14$ & $7_{b}, 10_{e}$ & $7,9-14$ \\
\hline 8 & $\mathbb{Z}_{8}$ & $11_{e}, 12_{e}$ & $11-17$ & $11_{e}, 12_{e}$ & $11-17$ \\
& $\mathbb{Z}_{4} \times \mathbb{Z}_{2}$ & $13_{e}, 14_{e}$ & $13-17$ & $13_{e}, 14 e$ & $12-17$ \\
& $\mathbb{Z}_{2}^{3}$ & $13_{e}, 16_{p}$ & $13,15-18$ & $15_{p}, 8_{b}$ & $8,12,14-18$ \\
& $Q_{8}$ & $11_{e}, 12_{e}$ & $11-17$ & $11_{e}, 12_{e}$ & $11-17$ \\
& $D_{4}$ & $11_{e}, 14_{p}$ & $11,13-17$ & $13_{p}, 12_{e}$ & $12-14$ \\
\hline 9 & $\mathbb{Z}_{9}$ & $13_{e}, 14_{e}$ & $13-16$ & $13_{e}, 14_{e}$ & $13-16$ \\
& $\mathbb{Z}_{3} \times \mathbb{Z}_{3}$ & $13_{e}, 14_{e}$ & $13-16$ & $9_{b}, 14_{e}$ & $9,13-16$ \\
\hline 10 & $\mathbb{Z}_{10}$ & $15_{e}, 14_{e}$ & $14-17$ & $13_{e}, 12_{e}$ & $12-17$ \\
& $D_{5}$ & $15_{e}, 16_{p}$ & $15-18$ & $15_{p}, 14_{e}$ & $16-20$ \\
\hline 11 & $\mathbb{Z}_{11}$ & $11_{b}, 16_{e}$ & $11,15-18$ & $15_{e}, 14_{e}$ & $15,17-18$ \\
\hline
\end{tabular}

The lower bounds on $k$ are given by:

$b$, the naive bound; $p$, self paired lemma; and e, exhaustive search. 
Table 5.2: Single Fixed Point Constructions' Greatest Lower Bounds on, and Spectra of $k$

\begin{tabular}{|c|c|c|c|c|c|}
\hline \multirow[t]{2}{*}{$v$} & \multirow[t]{2}{*}{ Group } & \multicolumn{2}{|c|}{ Entire- $k$} & \multicolumn{2}{|c|}{ Partial- $k$} \\
\hline & & $\begin{array}{c}\text { Lower Bounds } \\
\text { Odd,Even }\end{array}$ & $\begin{array}{c}\text { Existence } \\
\text { Spectra }\end{array}$ & $\begin{array}{c}\text { Lower Bounds } \\
\text { Odd,Even }\end{array}$ & $\begin{array}{c}\text { Existence } \\
\text { Spectra }\end{array}$ \\
\hline 3 & $\mathbb{Z}_{2}$ & $5_{b}, 6_{e}$ & $5-8$ & $5 b, 4 b$ & $4-8$ \\
\hline 4 & $\mathbb{Z}_{3}$ & $5_{b}, 6_{b}$ & $5-10$ & $7_{e}, 8_{e}$ & $7-10$ \\
\hline 5 & $\begin{array}{l}\mathbb{Z}_{4} \\
K_{4}\end{array}$ & $\begin{array}{c}7_{b}, 8_{e} \\
\gamma_{b}, 10_{p}\end{array}$ & $\begin{array}{c}7-10 \\
7,9-10\end{array}$ & $\begin{array}{l}7_{b}, 6_{b} \\
g_{p}, 8_{e}\end{array}$ & $\begin{array}{l}6-10 \\
8-10\end{array}$ \\
\hline 6 & $\mathbb{Z}_{5}$ & $g_{e}, 10_{e}$ & $9-12$ & $9_{p}, 10_{e}$ & $9-12$ \\
\hline 7 & $\begin{array}{l}\mathbb{Z}_{6} \\
S_{3}\end{array}$ & $\begin{array}{l}11_{e}, 10_{p} \\
11_{e}, 12_{p}\end{array}$ & $\begin{array}{l}10-14 \\
11-14\end{array}$ & $\begin{array}{c}11_{e}, 8_{b} \\
13_{e}, 12_{e}\end{array}$ & $\begin{array}{c}8,10-14 \\
12-14\end{array}$ \\
\hline 8 & $\mathbb{Z}_{7}$ & $9_{e}, 12_{e}$ & $9,11-15$ & $13_{e}, 12_{e}$ & $12-15$ \\
\hline 9 & $\begin{array}{c}\mathbb{Z}_{8} \\
\mathbb{Z}_{4} \times \mathbb{Z}_{2} \\
\mathbb{Z}_{2}^{3} \\
Q_{8} \\
D_{4} \\
\end{array}$ & $\begin{array}{l}13_{e}, 14_{e} \\
13_{e}, 14_{p} \\
15_{e}, 18_{p} \\
13_{e}, 14_{e} \\
13_{e}, 16_{p} \\
\end{array}$ & $\begin{array}{c}13-18 \\
13-18 \\
15,17,18 \\
13-18 \\
13,15-18 \\
\end{array}$ & $\begin{array}{l}13_{e}, 14 e \\
15_{e}, 14_{e} \\
17_{p}, 14 e \\
13_{e}, 14 e \\
15_{p}, 14 e \\
\end{array}$ & $\begin{array}{c}13-18 \\
14-18 \\
14,16-18 \\
13-18 \\
14-18 \\
\end{array}$ \\
\hline 10 & $\begin{array}{c}\mathbb{Z}_{9} \\
\mathbb{Z}_{3} \times \mathbb{Z}_{3}\end{array}$ & $\begin{array}{l}15_{e}, 16_{e} \\
15_{e}, 16_{e}\end{array}$ & $\begin{array}{l}15-22 \\
15-22\end{array}$ & $\begin{array}{l}15_{e}, 16_{e} \\
15_{e}, 14_{e}\end{array}$ & $\begin{array}{l}15-20 \\
14-20\end{array}$ \\
\hline 11 & $\begin{array}{l}\mathbb{Z}_{10} \\
D_{5}\end{array}$ & $\begin{array}{l}17_{e}, 16_{e} \\
17_{e}, 18_{p}\end{array}$ & $\begin{array}{l}16-22 \\
17-23\end{array}$ & $\begin{array}{l}17_{e}, 12_{e} \\
17_{p}, 16_{e}\end{array}$ & $\begin{array}{c}12,16-23 \\
18-23\end{array}$ \\
\hline 12 & $\mathbb{Z}_{11}$ & $15_{e}, 14 b$ & $17-24$ & $15_{e}, 16_{e}$ & $18-24$ \\
\hline 13 & $\begin{array}{c}\mathbb{Z}_{12} \\
\mathbb{Z}_{6} \times \mathbb{Z}_{2} \\
\mathbb{Z}_{3} \rtimes \mathbb{Z}_{4} \\
A_{4} \\
D_{6}\end{array}$ & $\begin{array}{l}15_{b}, 16_{p} \\
15_{b}, 18_{p} \\
15_{b}, 20_{p} \\
15_{b}, 18_{p} \\
15_{b}, 22_{p}\end{array}$ & $\begin{array}{c}19-27 \\
19,21-27 \\
20-27 \\
19,21-27 \\
19,21-27\end{array}$ & $\begin{array}{l}15_{p}, 14 b \\
17_{p}, 14 b \\
19_{p}, 14 b \\
19_{p}, 14 b \\
21_{p}, 14 b\end{array}$ & $\begin{array}{c}14,18-27 \\
20-27 \\
21-27 \\
20-27 \\
20,22-27\end{array}$ \\
\hline 14 & $\mathbb{Z}_{13}$ & $15_{b}, 16_{b}$ & $21-29$ & $15_{b}, 16_{b}$ & $19,21-29$ \\
\hline 15 & $\begin{array}{l}\mathbb{Z}_{14} \\
D_{7}\end{array}$ & $\begin{array}{l}17_{b}, 18_{p} \\
17_{b}, 24 p\end{array}$ & $\begin{array}{l}24-31 \\
25-31\end{array}$ & $\begin{array}{l}17_{b}, 16_{b} \\
23_{p}, 16_{b}\end{array}$ & $\begin{array}{l}24-31 \\
26-31\end{array}$ \\
\hline 16 & $\mathbb{Z}_{15}$ & $17_{b}, 18_{b}$ & $17,26-33$ & $17_{b}, 18_{b}$ & $27-33$ \\
\hline
\end{tabular}

The lower bounds on $k$ are given by:

$b$, the naive bound; $p$, self paired lemma; and e, exhaustive search. 
Table 5.3: Simple Double Point Constructions' Greatest Lower Bounds on, and Existence Spectra of $k$

\begin{tabular}{|c|c||c|c||c|c|}
\hline \multirow{2}{*}{$v$} & \multirow{2}{*}{ Group } & \multicolumn{2}{|c||}{ Entire- $k$} & \multicolumn{2}{c|}{ Partial- $k$} \\
\cline { 3 - 6 } & & $\begin{array}{c}\text { Lower Bounds } \\
\text { Odd,Even }\end{array}$ & $\begin{array}{c}\text { Existence } \\
\text { Spectra }\end{array}$ & $\begin{array}{c}\text { Lower Bounds } \\
\text { Odd,Even }\end{array}$ & $\begin{array}{c}\text { Existence } \\
\text { Spectra }\end{array}$ \\
\hline \hline 4 & $\mathbb{Z}_{2}$ & $9_{e}, 10_{p}$ & $9-13$ & $11_{e}, 10_{e}$ & $10-13$ \\
\hline 5 & $\mathbb{Z}_{3}$ & $11_{e}, 10_{p}$ & $10-14$ & $11_{e}, 10_{e}$ & $10-14$ \\
\hline 6 & $\mathbb{Z}_{4}$ & $13_{3}, 14_{e}$ & $13-17$ & $13_{e}, 12_{e}$ & $12-16$ \\
& $K_{4}$ & $13_{e}, 14_{p}$ & $13-17$ & $13_{p}, 12_{e}$ & $12-15$ \\
\hline 7 & $\mathbb{Z}_{5}$ & $13_{e}, 14 e$ & $13-18$ & $13_{e}, 14_{e}$ & $13-16$ \\
\hline 8 & $\mathbb{Z}_{6}$ & $15_{e}, 16 e$ & $15-18$ & $15_{e}, 14 e$ & $14-18$ \\
& $S_{3}$ & $13_{e}, 16_{p}$ & $13,15-18$ & $17_{e}, 16_{e}$ & $16-18$ \\
\hline 8 & $\mathbb{Z}_{7}$ & $13_{b}, 14_{p}$ & $16-18$ & $13_{b}, 14 b$ & \\
\hline 9 & $\mathbb{Z}_{8}$ & $13_{b}, 16_{p}$ & $18-20$ & $15_{p}, 14 b$ & \\
& $\mathbb{Z}_{4} \times \mathbb{Z}_{2}$ & $13_{b}, 18_{p}$ & $18-20$ & $17_{p}, 14 b$ & \\
& $\mathbb{Z}_{2}^{3}$ & $13_{b}, 22_{p}$ & & $21_{p}, 14 b$ & \\
& $Q_{8}$ & $13_{b}, 16_{p}$ & $17-20$ & $13_{b}, 14 b$ & \\
& $D_{4}$ & $13_{b}, 20_{p}$ & $19-20$ & $19_{p}, 14 b$ & \\
\hline 10 & $\mathbb{Z}_{9}$ & $15_{b}, 16_{p}$ & $19-22$ & $15_{b}, 14 b$ & \\
\hline
\end{tabular}

The lower bounds on $k$ are given by:

$b$, the naive bound; $p$, self paired lemma; and e, exhaustive search. 
Table 5.4: Augmented Double Point Constructions' Greatest Lower Bounds on, and Existence Spectra of $k$

\begin{tabular}{|c|c||c|c||c|c|}
\hline$v$ & \multirow{2}{*}{ Group } & \multicolumn{2}{c||}{ Entire- $k$} & \multicolumn{2}{c|}{ Partial- $k$} \\
\cline { 3 - 6 } & & $\begin{array}{c}\text { Lower Bounds } \\
\text { Odd,Even }\end{array}$ & $\begin{array}{c}\text { Existence } \\
\text { Spectra }\end{array}$ & $\begin{array}{c}\text { Lower Bounds } \\
\text { Odd,Even }\end{array}$ & $\begin{array}{c}\text { Existence } \\
\text { Spectra }\end{array}$ \\
\hline \hline 4 & $\mathbb{Z}_{2}$ & $7_{b}, 6_{b}$ & $6-10$ & $7_{b}, 6_{b}$ & $6-9$ \\
\hline 5 & $\mathbb{Z}_{3}$ & $7_{b}, 8_{b}$ & $7-11$ & $7_{b}, 8_{b}$ & $7-10$ \\
\hline 6 & $\mathbb{Z}_{4}$ & $9_{b}, 10_{p}$ & $9-13$ & $9_{b}, 10_{e}$ & $9-12$ \\
& $K_{4}$ & $9_{b}, 12_{p}$ & $9,11-14$ & $11_{b}, 10_{e}$ & $10-13$ \\
\hline 7 & $\mathbb{Z}_{5}$ & $11_{e}, 10_{b}$ & $11-14$ & $9_{b}, 10_{b}$ & $9-13$ \\
\hline 8 & $\mathbb{Z}_{6}$ & $13_{e}, 12_{p}$ & $12-15$ & $11_{p}, 12_{e}$ & $11-14$ \\
& $S_{3}$ & $13_{e}, 14_{p}$ & $13-16$ & $13_{p}, 14_{e}$ & $13-16$ \\
\hline 9 & $\mathbb{Z}_{7}$ & $13_{e}, 14_{e}$ & $13-18$ & $13_{e}, 14_{e}$ & $13-16$ \\
\hline 10 & $\mathbb{Z}_{8}$ & $15_{e}, 14_{p}$ & $14-18$ & $15_{e}, 16_{e}$ & $15-18$ \\
& $\mathbb{Z}_{4} \times \mathbb{Z}_{2}$ & $15_{e}, 16_{p}$ & $15-18$ & $15_{p}, 16_{e}$ & $15-18$ \\
& $\mathbb{Z}_{2}^{3}$ & $17_{e}, 20_{p}$ & & $19_{p}, 18_{e}$ & \\
& $Q_{8}$ & $15_{e}, 14_{p}$ & $14-18$ & $15_{p}, 14_{e}$ & $14-18$ \\
& $D_{4}$ & $15_{e}, 18_{p}$ & $15,17-18$ & $17_{p}, 16_{e}$ & $16-19$ \\
\hline
\end{tabular}

The lower bounds on $k$ are given by:

$b$, the naive bound; $p$, self paired lemma; and e, exhaustive search. 
Table 5.5: New Least Upper Bounds Found by the Constructions

\begin{tabular}{|c|c|c|c|c|}
\hline$v$ & $k$ & Construction & New Bound & Old Bound \\
\hline \hline 7 & 13 & Simple Double Fixed Point & 72 & 76 \\
\hline 8 & 13 & Simple Double Fixed Point & 85 & 92 \\
& 14 & Simple Double Fixed Point & 97 & 99 \\
& 15 & Simple Double Fixed Point & 97 & 105 \\
& 16 & Simple Double Fixed Point & 104 & 107 \\
\hline 9 & 16 & Simple Double Fixed Point & 120 & 129 \\
& 17 & Simple Double Fixed Point & 127 & 137 \\
& 18 & Simple Double Fixed Point & 134 & 141 \\
& 19 & Simple Double Fixed Point & 141 & 144 \\
\hline 10 & 13 & Single Fixed Point & 127 & 136 \\
& 17 & Simple Double Fixed Point & 144 & 152 \\
& 18 & Simple Double Fixed Point & 152 & 155 \\
\hline 11 & 19 & Simple Double Fixed Point & 179 & 191 \\
& 20 & Simple Double Fixed Point & 188 & 201 \\
\hline 12 & 17 & Single Fixed Point & 188 & 199 \\
\hline 13 & 18 & Single Fixed Point & 217 & 237 \\
& 19 & Single Fixed Point & 229 & 240 \\
& 20 & Single Fixed Point & 241 & 244 \\
\hline 14 & 19 & Single Fixed Point & 248 & 252 \\
& 21 & Single Fixed Point & 274 & 277 \\
& 22 & Single Fixed Point & 287 & 300 \\
\hline 15 & 25 & Single Fixed Point & 351 & 365 \\
\hline 16 & 25 & Single Fixed Point & 391 & 421 \\
& 27 & Single Fixed Point & 406 & 421 \\
\hline
\end{tabular}


are noted in Table 5.5. The new bounds come from both the single fixed point constructions and the Entire- $k$ simple double fixed point construction. The use of groups other than the cyclic groups do not lower the $k$ value in most cases. One systematic exception is by the Partial- $k$ no fixed point construction where the groups are direct products of a prime cyclic group. These groups are $K_{4}, \mathbb{Z}_{2}^{3}$, and $\mathbb{Z}_{3} \times \mathbb{Z}_{3}$. Orthogonal arrays are constructed for parameters where orthogonal arrays are known to already exist. Thus these groups produce no new least upper bounds. More insight into the orthogonal arrays produced is given in subsection 5.3.3.

More notably the non-cyclic groups $S_{3}, Q_{8}$ and $\mathbb{Z}_{3} \times \mathbb{Z}_{3}$ provided new least upper bounds in three cases where the cyclic groups fail to produce a cover starter to achieve the bound. The case with the $\mathbb{Z}_{3} \times \mathbb{Z}_{3}$ is by the Partial- $k$ single fixed point construction with $v=10$ and $k=13$. The other two cases are obtained by the Entire- $k$ simple double fixed point construction $G=S_{3}$ when $k=13$ and $v=8$ and for $G=Q_{8}, k=17$ and $v=10$. Note that for the cover starter found for $Q_{8}$ exhaustive searching was not performed.

\subsection{Comparison of Size and Lower Bounds Found for Covering Arrays Produced by Each Construction}

The eight constructions that are examined in this thesis produce covering arrays of varying size for a given $v$ and $k$. In this section these differing sizes of $N$ are compared. The value of $N$ is treated as a measure of efficiency; the 
lower the values of $N$ for a given $v$ and $k$ the more efficient the construction is and the better a choice for testing applications. This comparison provides the information for establishing a procedure to use when searching for new least upper bounds on covering arrays with these constructions. Note that the discussion does not take into account the orthogonal arrays found. The orthogonal arrays exist isolated from the rest of a spectrum for a given construction and group. As well, the orthogonal arrays produced do not yield new least upper bounds on covering arrays.

The sizes of $N$ for each construction are listed in Table 5.6. Table 5.7 is a simplified comparison of the existences which compares the no fixed point, single fixed point, simple double fixed point, and augmented double fixed point constructions. The Entire- $k$ and Partial- $k$ constructions of each type are grouped together as the size of the arrays they produce are the same or similar for given $v$ and $k$. The most efficient construction for a given $v$ and $k$, which is the one with the lowest $N$, is in bold. If no construction is bold faced in a cell then those constructions fail to reduce the $N$ achieved by a construction of a greater $k$ and/or $v$.

Table 5.7 shows the simple double fixed point is most efficient construction. The only entry that is not bold faced is for $v=8$ and $k=12$. Only the Partial- $k$ style is found here which produces the same size covering array as the Entire- $k$ style with $v=8$ and $k=13$.

The single fixed point construction is the next most efficient in the table. It is only bested by the simple double fixed point constructions. Also it is the only other construction to yield new least upper bounds.

Although the no fixed point constructions failed to yield new least upper 
Table 5.6: The Number of Columns Produced, $N$, by Each Cover Starter Construction

\begin{tabular}{|l|l|c|}
\hline Construction & Style & $N$ \\
\hline \hline No Fixed Points & Entire- $k$ & $k v$ \\
No Fixed Points & Partial- $k$ & $k v$ \\
\hline Single Fixed Point & Entire- $k$ & $k(v-1)+1$ \\
Single Fixed Point & Partial- $k$ & $k(v-1)+1$ \\
\hline Simple Double Fixed Point & Entire- $k$ & $k(v-2)+\mathcal{N}_{k}$ \\
Simple Double Fixed Point & Partial- $k$ & $(k+1)(v-2)+\mathcal{N}_{k}$ \\
\hline Augmented Double Fixed Point & Entire- $k$ & $(k+\phi)(v-2)+\mathcal{N}_{k}$ \\
Augmented Double Fixed Point & Partial- $k$ & $(k+\phi)(v-2)+\mathcal{N}_{k}$ \\
\hline
\end{tabular}

$\mathcal{N}_{k}=C A N(2, k)$ and $\phi=3$ or 4

bounds for the values $v$ and $k$ searched there is an instance where it did find a lower $N$ for a given $v$ and $k$ then the other constructions. This case is where $v=10, G=\mathbb{Z}_{10}$, and $k=12$. Also note in the regions searched the augmented double fixed point constructions can be found for several values of $k$ lower than the simple double fixed point construction for a given $v$, thus proving that they are worth further investigation.

The constructions are now compared arithmetically to give the order of the constructions to use when searching for new least upper bounds for values of $v$ and $k$ not proven by this thesis. The basis of the comparison is the difference between the values of $N$ by the constructions for a given $k$ and $v$. The value $\alpha$ is denotes as how much lower of a value of $k$ is needed for a given $v$ so that a less efficient construction is able to produce a $N$ less than the $N$ of a more efficient construction that can produce a covering array for $v$ and $(k+\alpha)$. 

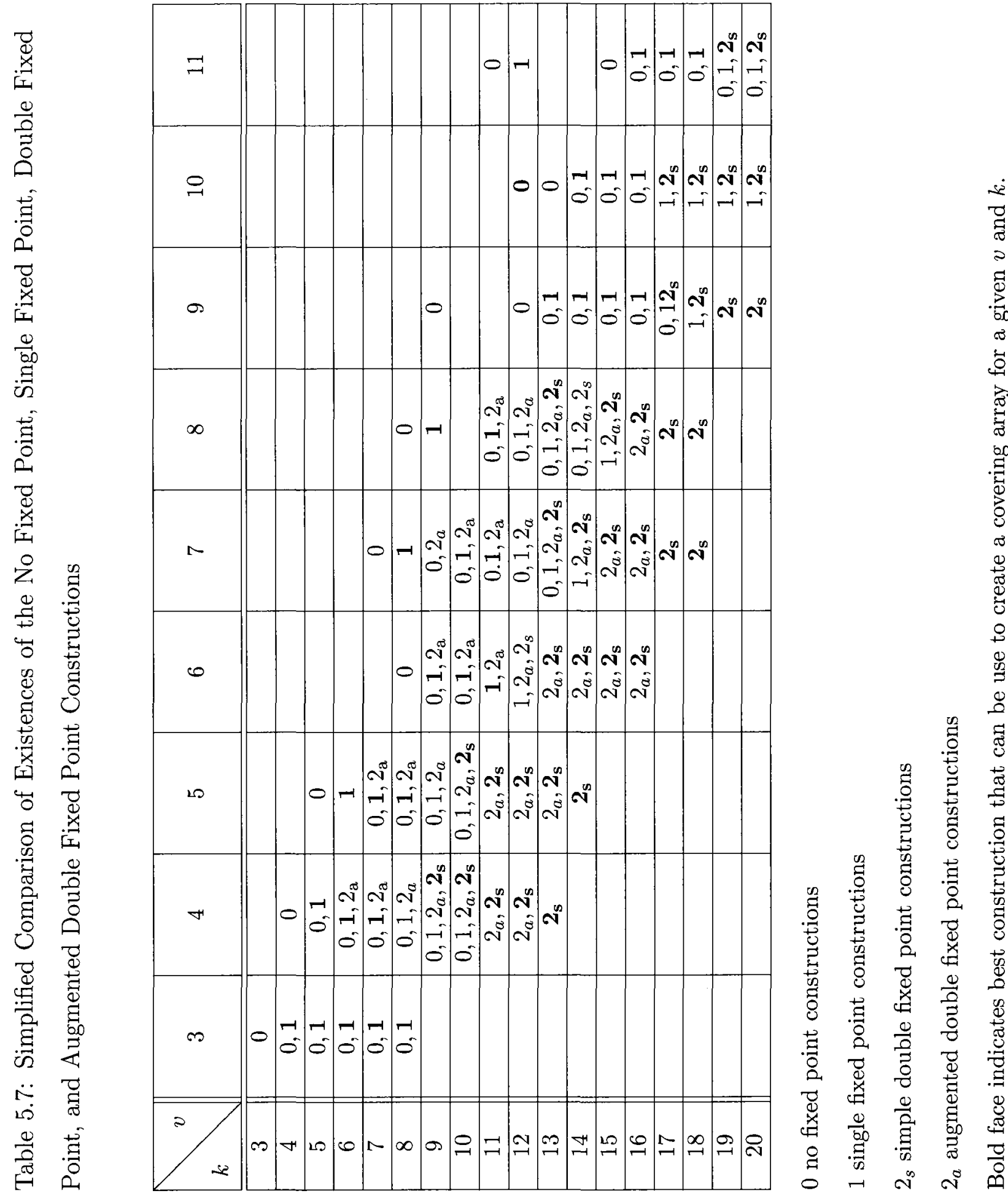
The sizes of the covering arrays produced by the different constructions are given in Table 5.6. Naive bounds of the constructions are reiterated as they provide information use by the comparisons. The no fixed point constructions require $k \geq v$, the single fixed point require $k \geq v+1$, and the double fixed point constructions need $k \geq v+4$ for the simple and $k \geq v+2$ for the augmented. As well in order for there to be a non trivial group action $v \geq 2$ for the no fixed point, $v \geq 3$ for the single fixed point, and $v \geq 4$ for the double fixed point constructions.

The comparison showing the single fixed point produces a smaller $N$ than the no fixed point constructions for a given $v$ and $k$ is in Equation 5.1. Note that in all cases it can be assumed that $k \geq v \geq 1$.

$$
k(v-1)+1<k(v-1)+k=k v
$$

The no fixed point construction needs a starter to exist for a $k$ that is $\alpha$ less than the lowest existence of a value of $k$ for the single fixed point construction of $k$ for a given $v$ by Equation 5.2. Here, $k<v$ and as such $\alpha \geq 2$.

$$
\begin{gathered}
k v<(k+\alpha)(v-1)+1 \\
\frac{k}{v-1}<\alpha
\end{gathered}
$$

All of the double fixed point constructions rely on a $C A(N ; 2, k)$ such with $N=C A N(2, k)$. For the purpose of concise notation $\mathcal{N}_{k}$ is set such that $\mathcal{N}_{k}=C A N(2, k)$. The following relationship between $k$ and $\mathcal{N}_{k}$ was established by Katona[14]: 


$$
k=\left(\begin{array}{c}
\mathcal{N}_{k}-1 \\
\left\lfloor\mathcal{N}_{k}-1\right\rfloor
\end{array}\right) .
$$

The Entire- $k$ simple double fixed point is more efficient than the Partial- $k$ style for a given $v$ and $k$ and the augmented double fixed point constructions are larger than either of the simple constructions by Equation 5.3. The naive bounds on the simple double fixed point constructions give that where both the double fixed point constructions exist is when $k+4 \geq v \geq 4$. Recall that $\phi=3$ or 4.

$$
k(v-2)+\mathcal{N}_{k}<(k+1)(v-2)+\mathcal{N}_{k}<(k+\phi)(v-2)+\mathcal{N}_{k}
$$

It can also be gleaned from equation 5.3 that the Partial- $k$ simple double fixed point construction needs to exist where $k$ is 2 less, $\alpha=2$, than the Entire- $k$ equivalent in order to be a best construction. Note an augmented double fixed point would require $\alpha=4$ or 5 depending on the value of $\phi$ to better an Entire- $k$ construction for the same value of $v$.

Where the Entire- $k$ simple double fixed point exists it is most efficient by Equation 5.4. The relation of $\mathcal{N}_{k}$ to $k$ gives when $k \geq 8$ then $k>\mathcal{N}_{k}$. For the simple double fixed point cases $k \geq 8$ as $k+4 \geq v \geq 4$ due to the bounds on the construction.

$$
k(v-2)+\mathcal{N}_{k}<k(v-2)+k<k(v-1)+1<k v
$$

The $\alpha$ value required for single fixed point constructions to be more efficient then the lower bound of the spectra of the Entire- $k$ simple double fixed point is given in Equation 5.5.

$$
k(v-1)+1<(k+\alpha)(v-2)+\mathcal{N}_{k}
$$




$$
\frac{k-\mathcal{N}_{k}+1}{v-2}<\alpha
$$

The Partial- $k$ simple fixed point is the next best construction. This is easy to see by calculating, via Equation 5.6, the $\alpha$ comparing the construction to the single fixed point constructions, which are the next best after the simple double fixed point.

$$
\begin{gathered}
k(v-1)+1<(k+\alpha+1)(v-2)+\mathcal{N}_{k} \\
\frac{k-v-\mathcal{N}_{k}+3}{v-2}<\alpha
\end{gathered}
$$

Note $k+4 \geq v \geq 4$ and $k+1>\mathcal{N}_{k}$ for all $k>8$. As well $k$ grows at a factorial rate in comparison to $\mathcal{N}_{k}$. This gives that the left hand side of the equation is positive and $\alpha \geq 1$.

Equation 5.7 determines when the augmented double fixed point constructions are more efficient then the no fixed point.

$$
\begin{gathered}
k v<(k+\phi)(v+2)+\mathcal{N}_{k} \\
\frac{\phi v+\mathcal{N}_{k}-2 \phi}{2}<k
\end{gathered}
$$

Since $\mathcal{N}_{k}$ grows at inverse factorial to $k$ the equation, $\frac{\phi}{2} v<k$ can be used to estimate where, for high enough $k$, the augmented double fixed point is more efficient than the no fixed point construction. For $\phi=3$ this value is where $k$ is $\frac{3}{2}$. In the ranges of $v$ and $k$ that are searched in this thesis $\frac{3}{2} v=k$ is approximately to the cusp of the lower end of both of those constructions spectra of $k$ as seen in Table 5.7. Experimentally these two constructions existence spectra values extend to lower values of $k$ than the single fixed point or simple double fixed point. The no fixed point yields a covering 
array that is the most efficient of the eight constructions for $v=10$ and $k=12$.

The augmented double fixed point is estimated to overtake the single fixed point in efficiency when $k \geq \phi$ by Equation 5.8. From the data obtained by the searches the simple double fixed point constructions are expected to exist and best all other constructions for these values of $k$.

$$
\begin{gathered}
k(v-1)+1<(k+\phi)(v+2)+\mathcal{N}_{k} \\
\phi v+\mathcal{N}_{k}+1-2 \phi<k
\end{gathered}
$$

The sequence of constructions to use when searching for new least upper bounds is to first use the Entire- $k$ double fixed point by Equations 5.3 and 5.4 followed by the Partial- $k$ double fixed point by Equation 5.6. Next the single fixed point constructions by Equations 5.1 and 5.8. The no fixed point constructions follow unless the values of $v$ and $k$ reverse the inequality of Equation 5.7. In that case the simple double fixed point constructions should be used.

\subsection{Gaps in the Spectra of $k$ values for a Given Group and Construction}

\subsubsection{Introduction}

There are constructions that do not have continuous existence spectra of $k$ values for cover starters for given $v$ and $G$. Some of these spectra are missing a single $k$ value. Examples include the groups $K_{4}, \mathbb{Z}_{7}$, and $D_{4}$ by the Entire- $k$ 
single fixed point as seen in Table 5.2. Others discontinuities have a larger gaps between the values of $k$ such as $\mathbb{Z}_{10}$ and $\mathbb{Z}_{12}$ by the Partial- $k$ single fixed point.

These gaps in the values are largely accounted for by two arguments. One being the notion of self paired orbitals that yields new bounds on $k$ via Lemma 5.2 and the other is based on a discussion of patterns of orthogonal arrays existences.

Lemma 5.2 and the existence of orthogonal arrays reasonably account for all but two of the discontinuities. These peculiar cases are by the Partial- $k$ single fixed point construction where $v=14, k=20$, and $G=\mathbb{Z}_{13}$ and the Partial- $k$ no fixed point construction where $v=11, k=16$, and $G=$ $\mathbb{Z}_{11}$. Note due to the size of $v$ and $k$ these ranges were not exhaustively searched and are indefinite as to their non existence and for the groups and constructions where these two gaps are found no self pair orbitals are required to be contained by the cover starters. Thus every proven gap is accounted for by Lemma 5.2 and the orthogonal array existence patterns.

\subsubsection{Self Paired Orbitals}

The single gaps in a spectra of $k$ values, by and large, exist when the number of factors is even for the Entire- $k$ style constructions and is odd for the Partial- $k$ style constructions. Lemma 5.2 explains this phenomena. It gives a new bound on the length of a cover starter, $\kappa$, when $\kappa$ is even. This bound is forced by the number of self-paired orbitals, $b^{*}$, a given group has. The naive bound was simply $\kappa \geq b$, where $b$ is the number of orbitals the cover starter need to contain except for the simple double fixed point cases. There 
the naive bound was $\kappa \geq b+2$ as multiple occurrences of each fixed point are required.

Definition 5.1 (Self Paired Orbital). A self paired orbital, $B$, is one that for every pair $(x, y) \in B$ then the pair $(y, x) \in B$.

Lemma 5.2 (Even Length Cover Starter Lemma). Let there be a cover starter, $T$, of length $\kappa$ and a group, $G$. Let the number of orbitals the cover starter is required to cover be $b$ and let $b^{*}$ be the number of the orbitals that are self paired. If $2 \mid \kappa$ then $T$ is at least of length $b+b^{*}$.

Proof. A cover starter, $T$, is required to contain a representative pair of every required orbital at every distance $s$-apart for $\{1, \ldots,(\kappa-1)\}$ and when $2 \mid \kappa$ there exists a distance $\frac{\kappa}{2}$-apart. When a pair of distinct entries of $T,(x, y)$, is at distance $\frac{\kappa}{2}$-apart then the pair $(y, x)$ is at $-\frac{\kappa}{2}$-apart. Moreover the pair $(y, x)$ is at distance $\frac{\kappa}{2}$-apart, since $-\frac{\kappa}{2} \equiv \frac{\kappa}{2} \bmod m$. Thus when a self paired orbital has a representative pair, $(x, y)$, at $\frac{\kappa}{2}$-apart then there is an additional pair $(y, x)$ from the same orbital in the cover starter at distance $\frac{\kappa}{2}$-apart.

Recall the starter vector needs a representative pair from each required orbital at every distance $s$-apart $s=1, \ldots,(\kappa-1)$. A self paired orbital occupies at least two positions when $2 \mid \kappa$ for the distance apart $\frac{\kappa}{2}$. Thus at the distance $\frac{\kappa}{2}$-apart $b+b^{*}$ positions of the cover starter are needed to contain all the representative pairs required.

For all the constructions, except the two simple double fixed point cases, the new bound on $\kappa$, when $2 \mid \kappa$, is $\kappa \geq b+b^{*}$. For the simple double fixed point constructions the new bound is $\kappa \geq b+b^{*}+2$. 
Examining the orbital types shows how to find the self paired orbitals of a given group action. Recall the different types are shown in Table 4.1. Representative pairs of the Type I are not required to be contained by any cover starter. The Type II orbitals are easily seen to not be self paired. The Type III and IV orbitals can be represented in the form presented by Table 5.8. The Type III orbital is self paired by definition. If a Type IV orbital is self paired than it contains both pairs $\left(e, g_{i}\right)$ and $\left(g_{i}, e\right)$. That is $g_{i}$ is an element such that $g_{i}^{g_{i}}=e$. Since the action of $G$ used is the action of $G$ on itself by left multiplication the Type IV orbitals are self paired when $g_{i} g_{i}=e$. Thus $b^{*}$ is simply the number of order two elements of $G$ plus one for the type III orbital. This result gives that Lemma 5.2 directly accounts for all the single gaps given in Table 5.9.

Table 5.8: The Type III and IV Orbitals of a Regular Group Action.

$$
\begin{aligned}
B_{1} & =\left\{\left(e, g_{1}\right)^{G}\right\} \\
B_{2} & =\left\{\left(e, g_{2}\right)^{G}\right\} \\
B_{3} & =\left\{\left(e, g_{3}\right)^{G}\right\} \\
\vdots & \vdots \\
B_{|G|} & =\left\{\left(e, g_{|G|}\right)^{G}\right\}
\end{aligned}
$$

There are instances of single value $k$ gaps in the ranges $k$ for $2 \mid \kappa$ that occur just beyond the bound given by Lemma 5.2. This is a plausible consequence of the cover starter having effectively less positions to contain representative pairs due to the self paired orbitals. There are two instances of no fixed point constructions: $\mathbb{Z}_{4}, k=6$, by the Entire- $k$ case and $\mathbb{Z}_{11}, k=13$, by the 
Table 5.9: Gaps in Cover Starter Existence Spectra Directly Due to The Even Length Cover Starter Lemma 5.2

\begin{tabular}{|l|c|c|c|}
\hline Construction & $v$ & $G$ & $k$ \\
\hline \hline Entire- $k$ No Fixed Point & 8 & $D_{4}$ & 12 \\
\hline Partial- $k$ No Fixed Point & 8 & $\mathbb{Z}_{2}^{3}$ & 13 \\
\hline Entire- $k$ Single Fixed Point & 5 & $K_{4}$ & 8 \\
& 9 & $\mathbb{Z}_{2}^{3}$ & 16 \\
& 9 & $D_{4}$ & 14 \\
\hline Partial- $k$ Single Fixed Point & 9 & $\mathbb{Z}_{2}^{3}$ & 15 \\
\hline Entire- $k$ Simple Double Fixed Point & 5 & $K_{4}$ & 8 \\
\hline Entire- $k$ Augmented Double Fixed Point & 10 & $D_{4}$ & 16 \\
\hline
\end{tabular}

Partial- $k$. As well the Entire- $k$ single fixed point has three occurrences where no cover starter exists for $k$ equal to the bound given by the self pair orbitals: $\mathbb{Z}_{7}$ when $k=10, \mathbb{Z}_{6} \times \mathbb{Z}_{2}$ when $k=20$, and $A_{4}$ when $k=20$. Note that three cases where the groups are $\mathbb{Z}_{4}, \mathbb{Z}_{11}$, and $\mathbb{Z}_{7}$ cover starters are proven to not exist by exhaustive search and the two cases with the groups are $\mathbb{Z}_{6} \times \mathbb{Z}_{2}$ and $A_{4}$ where the gaps are not conclusive as only heuristic searches were performed in these ranges.

Many improved lower bounds on $k$ are found as well. Some of these bounds are listed in Table 5.1, 5.2, 5.3, and 5.4 when the bound shown has a subscript of $p$. 


\subsubsection{Analysis of Existences That Provide Orthogonal Arrays}

The parameters that yield orthogonal arrays explain many of the discontinuous spectra of $k$ for constructions by given groups. They account for all the non singular gaps in the spectra and the remaining proven single gaps that Lemma 5.2 can not explain.

First note that orthogonal arrays are only found via the single and no fixed point constructions. As stated in the previous section of this chapter, the Entire- $k$ simple double fixed point is the most efficient of the double fixed point constructions and it has $k \geq v+4$ as the naive lower bound on $k$ and $v \geq 4$ for there to be a non-trivial group action with two fixed points. These relations on $k$ and $v$ determine that $k \geq 8$ and $\mathcal{N}_{k} \geq 6$. These restrictions imply that at the naive lower bound for $k$ that

$$
N=k(v-2)+\mathcal{N}_{k}=(v+4)(v-2)+\mathcal{N}_{k}=v^{2}+2 v-8+\mathcal{N}_{k} .
$$

Since $\mathcal{N}_{k} \geq 6$ and $v \geq 4, N \geq v^{2}+6>v^{2}$.

A strength 2 orthogonal array with repetition number of 1 , by Definition 2.1, requires that $N=v^{2}$. Thus none of the double fixed point constructions yield orthogonal arrays since in all cases $N>v^{2}$.

An orthogonal array is formed when a cover starter is found at the naive lower bound of $k$ for the no fixed point and the single fixed point constructions. In the no fixed point cases the naive lower bound on $k$ is $k=v$ and thus the value of $N$ at this lower bound is such that $N=k v=v^{2}$. The single fixed point cases have the naive lower bound on $k$ as $v+1$. Hence at this bound $N=k(v-1)+1=(v+1)(v-1)+1=v^{2}$. 
Table 5.10 gives all the occurrences of the orthogonal arrays found by the searches and the pattern in which they occur. Orthogonal arrays are only found when $v$ is prime power. Lemma 5.2 gives that the cover starters of the Entire- $k$ constructions can only occur when $k$ is odd. This is reflected in the search results as cover starters producing orthogonal arrays by these constructions are only found for odd primes for the no fixed point construction when $k=v$ and even for the single fixed point $k=v+1$. The Partial- $k$ no fixed point produces orthogonal arrays for $v$ equal to every prime power tested. The Partial- $k$ single fixed point construction realizes an orthogonal array for $v$ equal to a prime. Theorems $5.3,5.4$ and 5.5 give the proofs that orthogonal arrays are always found in these three patterns. The other pattern is given as a conjecture.

Theorem 5.3. When $v$ is an odd prime there exists a length $\kappa=v$ Entire- $k$ No Fixed Point cover starter over the group $Z_{v}$, which can be used to construct $a C A\left(v^{2} ; v, v\right)$

Proof. Let $T$ be a $\kappa \times 1$ array define by

$$
t_{i} \equiv \sum_{j=0}^{i} j \quad \bmod v .
$$

We show that $T$ is a cover starter. Choose an arbitrary distance $s$-apart for some $s=1, \ldots,(\kappa-1)$. It is required to show that $\left\{t_{i+s}-t_{i} \mid i \in \mathbb{Z}_{v}\right\}=\mathbb{Z}_{v}$. This is true if, for any $x \in \mathbb{Z}_{v}, t_{i+s}-t_{i}=x$ is solvable for $i$. Manipulation of 
Table 5.10: The List of Where Cover Starters are Found that Produce Orthogonal Arrays via their Constructions

\begin{tabular}{|l|c|c|}
\hline Construction and Pattern & $v$ & $G$ \\
\hline \hline Entire- $k$ No Fixed Point & 3 & $\mathbb{Z}_{3}$ \\
$v=p, G=\mathbb{Z}_{p}$, and $2 \nmid p$ & 5 & $\mathbb{Z}_{5}$ \\
& 7 & $\mathbb{Z}_{7}$ \\
\hline Partial- $k$ No Fixed Point & 11 & $\mathbb{Z}_{11}$ \\
$v=p^{n}$ and $G=\mathbb{Z}_{p}^{n}$ & 3 & $\mathbb{Z}_{2}$ \\
& 4 & $\mathbb{Z}_{3}$ \\
& 5 & $K_{4}$ \\
& 7 & $\mathbb{Z}_{5}$ \\
& 8 & $\mathbb{Z}_{2}^{3}$ \\
& 9 & $\mathbb{Z}_{3} \times \mathbb{Z}_{3}$ \\
\hline Entire- $k$ Single Fixed Point & 11 & $\mathbb{Z}_{11}$ \\
$v=2^{n}$ and $G=\mathbb{Z}_{2^{n}-1}$ & 8 & $\mathbb{Z}_{3}$ \\
& 16 & $\mathbb{Z}_{15}$ \\
\hline Partial $k$ Single Fixed Point & 3 & $\mathbb{Z}_{2}$ \\
$v=p$ and $G=\mathbb{Z}_{p-1}$ & 5 & $\mathbb{Z}_{4}$ \\
& 7 & $\mathbb{Z}_{6}$ \\
& 11 & $\mathbb{Z}_{10}$ \\
& 13 & $\mathbb{Z}_{12}$ \\
\hline
\end{tabular}


the equations gives:

$$
\begin{aligned}
t_{i+d}-t_{i} & =\frac{(i+s)(i+s-1)}{2}-\frac{(i)(i-i)}{2} \\
& =\frac{s(s+2 i-1)}{2} .
\end{aligned}
$$

Because this $v$ is odd and prime, $x=\frac{1}{2} s(s+2 i-1)$ has the solution

$$
i=\frac{1}{2}\left(\frac{2 x}{s}+1-s\right) \text {. }
$$

This gives that every group difference of $\mathbb{Z}_{v}$ is present.

Theorem 5.4. When $v$ is prime power, $p^{n}$ there exists a length $\kappa=v-1$ Partial-k No Fixed Point cover starter over the group $\mathbb{Z}_{p}^{n}$, which can be used construct a $C A\left(v^{2} ; 2, v, v\right)$.

Proof. Let $v$ be a prime power and $\mathbb{F}_{v}$ be the finite field of order $v$. The additive group of this field is $\mathbb{Z}_{p}^{n}$. Let $\omega$ be a primitive element of $\mathbb{F}_{v}$ such that $\langle\omega\rangle=\mathbb{F}_{v}^{\times}$. Recall that a Partial- $k$ no fixed point construction requires all the Type IV orbitals covered which are the non-identity group differences and the cover starter must contain all but one elements of $X$, here the missed element is 0 .

Assign the entries of a $(v-1) \times 1$ array, $T$, as $t_{i}=\omega^{i}$.

This assignment of $T$ satisfies the condition that all the entries of $T$ are distinct.

Pick an arbitrary distance $s$-apart for some $s=1, \ldots,(v-2)$. Now at each distance $s$-apart there are the pairs of the form $\left(\omega^{i+s}, \omega^{i}\right)$. Thus the pairs at the $s$-apart give the differences:

$$
\omega^{i+s}-\omega^{i}=\omega^{i}\left(\omega^{s}-1\right)
$$


For any non-zero group difference $x \in X$ we can solve $x=\omega^{i}\left(\omega^{s}-1\right)$ for $i$ :

$$
i=\log _{w}\left(s\left(\omega^{s}-1\right)^{-1}\right)
$$

Because $\omega^{s-1} \neq 0$

Theorem 5.5. When $v$ is prime there exists a length $\kappa=v$ Partial-k Single Fixed Point cover starter over group $\mathbb{Z}_{v-1}$, which can be used to construct a $C A\left(v^{2} ; 2, v+1, v\right)$.

Proof. Let $v$ be prime and $\mathbb{F}_{v}=\mathbb{Z}_{v}$ be the finite field of order $v$. Let $\omega$ be a primitive element in $\mathbb{F}_{v}$ so we have that $\langle\omega\rangle=\mathbb{F}_{v}^{\times}$. Define a map

$$
\sigma: \mathbb{F}_{v} \rightarrow \mathbb{Z}_{v-1} \cup \infty
$$

by

$$
\sigma(x)= \begin{cases}\infty & \text { if } x=0 \\ \log _{\omega}(x) & \text { otherwise. }\end{cases}
$$

Now define the $v \times 1$ array, $u$, by $u_{i}=i \cdot 1$ for $0 \leq i<v, 1 \in \mathbb{F}_{v}$. We claim that $t_{i}:=\sigma\left(u_{i}\right) \forall i=1, \ldots, v$ defines a length $v$ Partial- $k$ Single Fixed Point cover starter over group $Z_{v-1}$.

Since $u$ has a single $0, T$ has an $\infty$ in a single position, and thus covers all Type II orbitals at every distance $s$-apart $\forall s=1, \ldots,(v-1)$.

By the pigeon hole principle it suffices to show that all pairs at an arbitrarily chosen distance $s$-apart for $s=1, \ldots,(v-1)$ which do not contain $\infty$ belong to distinct orbitals, in this case group differences. Suppose that two such pairs have the same group difference:

$$
t_{i+s}-t_{i}=t_{j+s}-t_{j}
$$


for $i \neq j \in \mathbb{Z}_{v}$. The inverse image of Equation 5.9 through $\sigma$ gives

$$
\begin{aligned}
u_{i+s} / u_{i} & =u_{j+s} / u_{j} \\
1+s / i & =1+s / j \\
s / i & =s / j \\
i & =j .
\end{aligned}
$$

Conjecture 5.6. When $v=2^{n}$ there exists a length $\kappa=v+1$ Entire- $k$ Single Fixed Point cover starter over the group $\mathbb{Z}_{v-1}$, which is used to construct a $C A\left(v^{2} ; 2, v+1, v\right)$

\subsection{Expected Existence of Starters}

As shown in Table 3.9 there are examples where it seems that a cover starter of length $\kappa$ for a given $G$ and $X$ can have one element added to produce a cover starter of length $\kappa+1$. The results in Tables 5.1, 5.2, 5.3, and 5.4 show many gaps are proven to exist in the spectra of $k$ that demonstrate that in general one can not show that if a cover starter exists for a given $v, k$ and $G$ for a construction then a cover starter for $k+1$ exists. Patterns of orthogonal arrays are found at the naive bound and experimental evidence shows that for the gap between the naive bound and the rest of the existences grows as $v$ increases. Thus the orthogonal arrays are treated as anomalies that are outside the normal range of the spectrum of the $k$ values. The strong relationship between parity of the length of a cover starter and its existence is given by Lemma 5.2. Yet it is interesting to note that if a cover starter 
exists for parameters for a given $k, v$, and $G$, and the construction does not result in an orthogonal array, then there is always a cover starter found for $k+2$. Thus the data gathered in this thesis strongly indicates the plausibility of Conjecture 5.7 .

Conjecture 5.7. If a cover starter of length $\kappa$ exists for a Entire- $k$ or Partial- $k$ style covering array construction that does not produce a orthogonal array, then there exists a cover starter of length $\kappa+2$ 


\section{Chapter 6}

\section{Conclusions and Future Work}

The testing of component-wise systems is a need in a number of fields and one of the most documented is software development $[5,7,17,29]$. In most cases it is infeasible to test every possible configuration and as such methods that consume less resources yet capture a large number of the errors in a system are needed. Covering arrays, $C A_{\lambda}(N ; t, k, v)$, cover all $t$-wise interactions $\lambda$ times. These arrays have been shown to locate a large percentage of errors $[5,7,17,29]$ and very few tests are needed compared to an exhaustive search.

Eight constructions for covering arrays of strength $2, t=2$, and repetition number $\lambda=1$ were described within. They all employ the $\left[M^{G}, C\right]$ format with a cover starter used to build $M$. The $\left[M^{G}, C\right]$ structure requires a permutation group, $G$, and two arrays $M$ and $C$ with $k$ rows. $M$ is acted on by the group generating $|G|$ arrays that are concatenated together forming $M^{G}$. Six of the constructions have the array $C$ concatenated to $M^{G}$ to form $\left[M^{G}, C\right]$. This $\left[M^{G}, C\right]$ format is utilized by Meagher and Stevens along with employing a cover starter $[19,20,21]$, which is a $\kappa \times 1$ array that is 
used to build the $M$ of the $M^{G}$. The search for a cover starter is carried out via algorithmic means and thus this method for generating covering arrays is a hybrid of algebraic and algorithmic search techniques. Colbourn et al. use an adapted cover starter technique to create covering arrays of the same parameters as Meagher and Stevens [10]. In this thesis there are four constructions of each of the Entire- $k$ and the Partial- $k$ style utilized. The Entire- $k$ constructions are based on Meagher and Stevens construction and the Partial- $k$ are based on Colbourn et al.'s construction. The Partial- $k$ cover starters are required to contain a certain number of elements from the set. Three of the Partial- $k$ constructions require the entire set and in these cases the cover starter is termed a distinct cover starter.

Each construction style has four types: zero fixed point, single fixed point, simple double fixed point, and augmented double fixed point. The group action employed by a construction is regular on $|G|$ elements of a set and fixes the remaining elements. The number of fixed points used by a construction is indicated in the name. The Entire- $k$ and Partial- $k$ single fixed point constructions were previously developed and used by Meagher and Stevens and Colbourn et al. respectively. The other six constructions are new and introduced in this thesis. The simple double fixed point constructions produce the most efficient cover arrays of all of the constructions for a given $v$ and $k$ as they produce covering arrays with the smallest $N$. The zero fixed point and augmented double fixed point constructions are less efficient than both the single fixed point and the simple double fixed point types but are capable of producing covering arrays for lower values of $k$ for a given $v$ and show potential for achieving new least upper bounds on covering arrays in these 
lower ranges of $k$ for higher values of $v$ than were tested in this thesis.

Previously only the single fixed point Entire- $k$ and Partial- $k$ constructions were known and these only applied with cyclic groups [10, 19, 20, 21]. In this thesis the single fixed point constructions were examined with a greater range of exhaustive searches. In addition, all groups of a given size were utilized in all constructions. The exhaustive searches for the cover starters was performed via a Gray Code [15] and the reductions on the search spaces for each constructions' cover starter are given in Table 4.22. Beyond the feasible range of exhaustive searches heuristic hill climbs were performed to find cover starters for the zero fixed point, single fixed point, and simple double fixed point constructions.

New least upper bounds on covering arrays were found and a deepened knowledge of the existence behaviour of cover starters was obtained. The existence spectra of cover starters of a construction by a group give tighter lower bounds on $k$ by Lemma 5.2. Orthogonal arrays are found in patterns for the no fixed point and single fixed point constructions. New least upper bounds are achieved by the single fixed point and no fixed point constructions; three new least upper bounds are found via non-cyclic groups. For higher values of $v$ an efficient procedure is described for using the eight constructions to procure new least upper bounds by future work. 


\subsection{Contributions}

This thesis contributes many results useful to studying covering arrays and cover starter constructions:

- New least upper bounds on $C A N_{1}(N ; 2, k, v)$.

- Six new constructions for covering arrays.

- Expanded data via in-depth searching, different groups, and new constructions.

- New lower bounds on cover starter existences using the effect of self paired orbitals.

- Proofs that cover starter constructions yield orthogonal arrays.

The new least upper bounds are summarized in Table 5.5. New bounds were only achieved by the single fixed point constructions and the Entire- $k$ style of the simple double fixed point.

The data collected gives much insight on cover starter constructions. The use of additional groups yields three new least upper bounds. The three alternate groups that obtained these bounds were $S_{3}, Q_{8}$ and $\mathbb{Z}_{3} \times \mathbb{Z}_{3}$. Tables 5.1, $5.2,5.3$, and 5.4 give a comprehensive overview of the spectra and bounds on $k$.

The analysis of the gaps in the spectra of $k$ lead to Lemma 5.2. This lemma explains many gaps in the spectra and gives new greatest lower bounds on $k$ for a given construction and group. 
Table 6.1: The Patterns of Orthogonal Array Existences Occurring in the No Fixed Point and Single Fixed Point Construction Types

\begin{tabular}{|l|c|}
\hline Construction & Pattern \\
\hline \hline Entire- $k$ no fixed point & $v=p, G=\mathbb{Z}_{p}$, and $2 \nmid p$ \\
\hline Partiall $k$ no fixed point & $v=p^{n}$ and $G=\mathbb{Z}_{p}^{n}$ \\
\hline Partial $k$ single fixed point & $v=p$ and $G=\mathbb{Z}_{p-1}$ \\
\hline
\end{tabular}

Orthogonal arrays were found in four patterns highlighted in Table 6.1. The no fixed point constructions' and Partial- $k$ single fixed point construction's pattern are proven to always exist.

\subsection{Future Work}

Presented here is future work that arises from the results of this thesis:

- The fourth pattern possible pattern of orthogonal arrays presented in Conjecture 5.6 which the case where $v=2^{n}$ and $G=\mathbb{Z}_{2^{n}-1}$ for Entire- $k$ single fixed point could be resolved by finding a group, $H$ other than $G$, whose action on the set provides a way of generating a cover starter. That is this second group action of $H$ on a set of size $v$ has a way of generating a $\kappa \times 1$ array which is isomorphic to a cover starter for the Entire- $k$ single fixed point construction by $\mathbb{Z}_{2^{n}-1}$.

- Conjecture 5.7 postulates that if a cover starter of a construction by a group for a given $v$ and $k$ does not produce an orthogonal array then a cover starter exists for $k+2$. When a cover starter which does not 
produce an orthogonal array exists there is repetition of coverage; this ensured repetition in coverage could be use with a counting argument to give the existence result sought by the conjecture.

- Searches for the the eight constructions could be used to produce least upper bounds on covering arrays for higher values of $v$. The procedure outlined in Section 5.4 provides an order of which use of the constructions.

- The cover starters are used to form circulant arrays. The circulant is formed by applying a modular group action on the indices of the cover starter. Forming arrays with the actions of non-cyclic groups could yield cover starters with new least upper bounds. An example of this kind of alternate construction is given in Table 6.2 that is isomorphic to the orthogonal array given in Table 2.2.

The equivalence between the array in Table 2.2 and the one in Table 6.2 is given by the following: the columns are permuted via (1 1613118 $25)(31074151296$ )(14), the rows are permuted via (1 54432 ), and a set isomorphism $\sigma$ between $X=\{1,2,3,4\}$ and $Y=\{\infty, 0,1,2\}$ such that $\sigma(1)=\infty, \sigma(2)=0, \sigma(3)=1$, and $\sigma(4)=2$. The array in Table 6.2 can be seen to be a Partial- $k$ single fixed point construction with the cover starter $\{\infty, 2,0,1\}$ developed into a $(k-1) \times(k-1)$ array by the action of $K_{4}$ on its indices. The action on the array $M$ is by $\mathbb{Z}_{3}$. 
Table 6.2: A Partial- $k$ single fixed point construction with $v=4, k=5$, and $G=\mathbb{Z}_{3}$ and the cover starter's indices acted on by $K_{4}$ instead of a cyclic action.

$$
\left(\begin{array}{ccccc|ccccc|ccccc|c}
\infty & 2 & 0 & 1 & 0 & \infty & 0 & 1 & 2 & 1 & \infty & 1 & 2 & 0 & 2 & \infty \\
2 & \infty & 1 & 0 & 0 & 0 & \infty & 2 & 1 & 1 & 1 & \infty & 0 & 2 & 2 & \infty \\
1 & 0 & 2 & \infty & 0 & 2 & 1 & 0 & \infty & 1 & 0 & 2 & 1 & \infty & 2 & \infty \\
0 & 1 & \infty & 2 & 0 & 1 & 2 & \infty & 0 & 1 & 2 & 0 & \infty & 1 & 2 & \infty \\
0 & 0 & 0 & 0 & \infty & 1 & 1 & 1 & 1 & \infty & 2 & 2 & 2 & 2 & \infty & \infty
\end{array}\right)
$$

- The construction in the proof of Theorem 5.5 is very similar to Welch's construction for Costas arrays [9, 26]. Using cover starter like constructions could be used to create other combinatorial designs or designs of similar nature. For instance an increase of $k$ for given parameters of a Costas array will no longer be orthogonal and there will repetition of pairs. The construction would no longer yield Costas arrays but the result would be arrays with Costas array like properties with small amounts of repetition.

- Other meta-heuristics search techniques could be used such as simulated annealing, tabu search, and genetic algorithms. These searches may produce cover starters for values of $k$ that the hill climb did not in the ranges where exhaustive search is infeasible.

- Similar methods could be used to search for higher strength, $t \geq 3$, cover starters. 


\section{Appendix A}

\section{Cover Starter Examples}

The cover starter examples are given in this appendix. The number of factors, $k$, are in the first column, the groups, $G$, in the second column, and the final column has the groups generators and assignments of the group elements to digits and the cover starters. The cyclic groups are assigned to the expected elements of $\mathbb{Z}_{|G|}$ by addition. 
Table A.1: Entire- $k$ No Fixed Points Cover Starters

\begin{tabular}{|c|c|c|c|}
\hline $\mathrm{v}$ & $\mathrm{k}$ & $G$ & \\
\hline \multirow[t]{5}{*}{2} & & $\mathbb{Z}_{2}$ & \\
\hline & 3 & & 100 \\
\hline & 4 & & 1000 \\
\hline & 5 & & 10000 \\
\hline & 6 & & 1000000 \\
\hline \multirow[t]{7}{*}{3} & & $\mathbb{Z}_{3}$ & \\
\hline & 3 & & 100 \\
\hline & 4 & & 1000 \\
\hline & 5 & & 10000 \\
\hline & 6 & & 100000 \\
\hline & 7 & & 1000000 \\
\hline & 8 & & 10000000 \\
\hline \multirow[t]{6}{*}{ 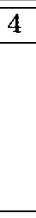 } & & $\mathbb{Z}_{4}$ & \\
\hline & 5 & & 21200 \\
\hline & 7 & & 23100000 \\
\hline & 8 & & 231000000 \\
\hline & 9 & & 231000000 \\
\hline & 10 & & 2310000000000 \\
\hline \multirow[t]{5}{*}{ 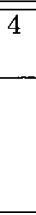 } & & $\overline{K_{4}}$ & $\begin{array}{l}\left\{x, y \mid x^{2}=y^{2}=i, x y=y x\right\} \\
0=i, 1=x, 2=y, 3=x y\end{array}$ \\
\hline & 7 & & 2031000 \\
\hline & 8 & & 203100000 \\
\hline & 9 & & 2031000000 \\
\hline & 10 & & 20310000000 \\
\hline \multirow[t]{6}{*}{5} & & $\mathbb{Z}_{5}$ & \\
\hline & 5 & & 13100 \\
\hline & 7 & & 1310000 \\
\hline & 8 & & 131000000 \\
\hline & 9 & & 131000000 \\
\hline & 10 & & 131000000000 \\
\hline \multirow[t]{6}{*}{6} & & $\mathbb{Z}_{6}$ & \\
\hline & 9 & & 531251000 \\
\hline & 10 & & 4305100000 \\
\hline & 11 & & 413410000000 \\
\hline & 12 & & 413410000000 \\
\hline & 13 & & 4134100000000 \\
\hline \multirow[t]{6}{*}{ 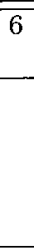 } & & $\overline{\overline{S_{3}}}$ & $\begin{array}{l}\left\{x, y \mid x^{3}=y^{2}=i, x^{-1}=y x y\right\} \\
0=i, 1=x, 2=x^{2}, 3=y, 4=x^{2} y, 5=x y\end{array}$ \\
\hline & 9 & & 134151000 \\
\hline & 10 & & 4124311000 \\
\hline & 11 & & 13415100000 \\
\hline & 12 & & 421053000000 \\
\hline & 13 & & 1341510000000 \\
\hline
\end{tabular}


Table A.1: Entire- $k$ No Fixed Points Cover Starters (continued)

\begin{tabular}{|c|c|c|c|}
\hline $\mathrm{v}$ & $\mathrm{k}$ & $G$ & \\
\hline \multirow[t]{8}{*}{7} & & $\mathbb{Z}_{7}$ & \\
\hline & 7 & & 1363100 \\
\hline & 9 & & 163361000 \\
\hline & 10 & & 4505151000 \\
\hline & 11 & & 14224100000 \\
\hline & 12 & & 4031561000000 \\
\hline & 13 & & 5236610000000 \\
\hline & 14 & & 52366100000000 \\
\hline \multirow[t]{8}{*}{8} & & $\mathbb{Z}_{8}$ & \\
\hline & 11 & & 43673417100 \\
\hline & 12 & & 671304610000 \\
\hline & 13 & & 6137564100000 \\
\hline & 14 & & 56205471000000 \\
\hline & 15 & & 562054710000000 \\
\hline & 16 & & 5620547100000000 \\
\hline & 17 & & 34263371000000000 \\
\hline \multirow[t]{6}{*}{$\overline{8} 8$} & & $\mathbb{Z}_{4} \times \mathbb{Z} 2$ & $\begin{array}{l}\left\{x, y \mid x^{4}=y^{2}=i, x y=y x\right\} \\
0=i, 1=x, 2=x^{2}, 3=x^{3}, 4=y, 5=y x, 6=y x^{2}, 7=y x^{3}\end{array}$ \\
\hline & 13 & & 7441620710000 \\
\hline & 14 & & 57014261000000 \\
\hline & 15 & & 241566100000000 \\
\hline & 16 & & 2415661000000000 \\
\hline & 17 & & 24156610000000000 \\
\hline \multirow[t]{6}{*}{$\overline{8}$} & & $\overline{\mathbb{Z}_{2}^{3}}$ & $\begin{array}{l}\left\{x, y, z \mid x^{2}=y^{2}=i, x y=y x, x z=z x, z y=y z,\right\} \\
0=i 1=x, 2=y, 3=x y, 4=z, 5=x z, 6=y z, 7=x y z\end{array}$ \\
\hline & 13 & & 4365120710000 \\
\hline & 15 & & 436512207100000 \\
\hline & 16 & & 6643052671000000 \\
\hline & 17 & & 64351271000000000 \\
\hline & 18 & & 643512710000000000 \\
\hline \multirow[t]{7}{*}{$\overline{8}$} & & $\overline{D_{4}}$ & $\begin{array}{l}\left\{x, y \mid x^{4}=y^{2}=i, x^{-1}=y x y\right\} \\
0=i, 1=x, 2=x^{2}, 3=x^{3}, 4=y, 5=x^{3} y, 6=x^{2} y, 7=x y\end{array}$ \\
\hline & $\overrightarrow{11}$ & & 12573617100 \\
\hline & 13 & & 5573730710000 \\
\hline & 14 & & 25603647710000 \\
\hline & 15 & & 164252710000000 \\
\hline & 16 & & 6725403100000000 \\
\hline & 17 & & 16425271000000000 \\
\hline \multirow[t]{8}{*}{$\overline{8}$} & & $\overline{\bar{Q}}$ & $\begin{array}{l}\left\{x, y \mid x^{4}=y^{4}=i, y x y=x^{3}\right\} \\
0=i, 1=x, 2=x^{2}, 3=x^{3}, 4=y, 5=x^{3} y, 6=x^{2} y, 7=x y\end{array}$ \\
\hline & 11 & & 37056317100 \\
\hline & 12 & & 274372471000 \\
\hline & 13 & & 4263057100000 \\
\hline & 14 & & \\
\hline & 15 & & 726430710000000 \\
\hline & 16 & & 7264307100000000 \\
\hline & 17 & & 72643071000000000 \\
\hline
\end{tabular}


Table A.1: Entire- $k$ No Fixed Points Cover Starters (continued)

\begin{tabular}{|c|c|c|c|}
\hline $\mathrm{v}$ & $\mathrm{k}$ & $G$ & \\
\hline \multirow[t]{5}{*}{9} & & $\overrightarrow{\mathbb{Z}_{9}}$ & \\
\hline & 13 & & 6124260510000 \\
\hline & 14 & & 46073057810000 \\
\hline & 15 & & 315278351000000 \\
\hline & 16 & & 7465016710000000 \\
\hline \multirow[t]{7}{*}{9} & & $\overline{Z_{3} \times Z_{3}}$ & $\left\{x, y \mid x^{3}=y^{3}=i, x y=y x\right\}$ \\
\hline & & & $0=i, 1=x, 2=x^{2}, 3=y, 4=x y$, \\
\hline & & & $5=x y^{2}, 6=, 7 x y=, 8=x^{2} y^{2}$ \\
\hline & 13 & & 1853235810000 \\
\hline & 14 & & 60570837810000 \\
\hline & 15 & & 836177881000000 \\
\hline & 16 & & 8637107810000000 \\
\hline \multirow[t]{5}{*}{$\overline{10}$} & & $\overline{\mathbb{Z}_{10}}$ & \\
\hline & 14 & & 40575189358100 \\
\hline & 15 & & 618936686100000 \\
\hline & 16 & & 7045610839100000 \\
\hline & 17 & & 27851064710000000 \\
\hline \multirow[t]{7}{*}{10} & & $\overline{D_{5}}$ & $\left\{x, y \mid x^{5}=y^{2}=i, x^{-1}=y x y\right\}$ \\
\hline & & & $0=i, 1=x, 2=x^{2}, 3=x^{3}, 4=x^{4}$, \\
\hline & & & $5=y, 6=x^{4} y, 7=x^{3} y, 8=x^{2} y, 9=x y$ \\
\hline & 15 & & 665212879918680 \\
\hline & 16 & & 3196951781150870 \\
\hline & 17 & & 70918568355034369 \\
\hline & 18 & & 798830892303700445 \\
\hline \multirow[t]{6}{*}{$\overline{11}$} & & $\overline{\mathbb{Z}_{11}}$ & \\
\hline & 11 & & 136041063100 \\
\hline & 15 & & 816181618532235 \\
\hline & 16 & & 069599110851013901 \\
\hline & 17 & & 28165256036095557 \\
\hline & 18 & & 1278890091094374409 \\
\hline
\end{tabular}


Table A.2: Partial- $k$ No Fixed Point Cover Starters

\begin{tabular}{|c|c|c|c|}
\hline $\mathrm{v}$ & $\mathrm{k}$ & $G$ & \\
\hline \multirow[t]{6}{*}{2} & & $\mathbb{Z}_{2}$ & \\
\hline & 2 & & 1 \\
\hline & 3 & & 10 \\
\hline & 4 & & 100 \\
\hline & 5 & & 1000 \\
\hline & 6 & & 100000 \\
\hline \multirow[t]{7}{*}{3} & & $\mathbb{Z}_{3}$ & \\
\hline & 3 & & 21 \\
\hline & 4 & & 221 \\
\hline & 5 & & 1210 \\
\hline & 6 & & 12100 \\
\hline & 7 & & 121000 \\
\hline & 8 & & 1210000 \\
\hline \multirow[t]{6}{*}{4} & & $\mathbb{Z}_{4}$ & \\
\hline & 6 & & 23100 \\
\hline & 7 & & 231000 \\
\hline & 8 & & 2310000 \\
\hline & 9 & & 23100000 \\
\hline & 10 & & 231000000 \\
\hline \multirow[t]{7}{*}{ 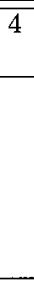 } & & $\overline{K_{4}}$ & $\begin{array}{l}\left\{x, y \mid x^{2}=y^{2}=i, x y=y x\right\} \\
0=i, 1=x, 2=y, 3=x y\end{array}$ \\
\hline & 4 & & 321 \\
\hline & 6 & & 23131 \\
\hline & 7 & & 121310 \\
\hline & 8 & & 2031000 \\
\hline & 9 & & 203100000 \\
\hline & 10 & & 203100000 \\
\hline \multirow[t]{6}{*}{5} & & 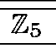 & \\
\hline & 5 & & 2431 \\
\hline & 7 & & 423410 \\
\hline & 8 & & 3234100 \\
\hline & 9 & & 20341000 \\
\hline & 10 & & 203410000 \\
\hline \multirow[t]{7}{*}{6} & & $\overline{\mathbb{Z}_{6}}$ & \\
\hline & 8 & & 3541200 \\
\hline & 9 & & 23415100 \\
\hline & 10 & & 234151000 \\
\hline & 11 & & 2341510000 \\
\hline & 12 & & 34205100000 \\
\hline & 13 & & 342051000000 \\
\hline \multirow[t]{5}{*}{$\overline{6}$} & & $\overline{S_{3}}$ & $\begin{array}{l}\left\{x, y \mid x^{3}=y^{2}=i, x^{-1}=y x y\right\} \\
0=i, 1=x, 2=x^{2}, 3=y, 4=x^{2} y, 5=x y\end{array}$ \\
\hline & 10 & & 143251000 \\
\hline & 11 & & 1432510000 \\
\hline & 12 & & 14325100000 \\
\hline & 13 & & 143251000000 \\
\hline
\end{tabular}


Table A.2: Partial- $k$ No Fixed Point Cover Starters (continued)

\begin{tabular}{|c|c|c|c|}
\hline $\mathrm{v}$ & $\mathrm{k}$ & $G$ & \\
\hline \multirow[t]{8}{*}{7} & & $\overline{\mathbb{Z}_{7}}$ & \\
\hline & 7 & & 326451 \\
\hline & 9 & & 42163561 \\
\hline & 10 & & 542236661 \\
\hline & 11 & & 3452646100 \\
\hline & 12 & & 45203661000 \\
\hline & 13 & & 45325610000000 \\
\hline & 14 & & 4532561000000 \\
\hline \multirow[t]{8}{*}{8} & & $\mathbb{Z}_{8}$ & \\
\hline & 11 & & 2554266371 \\
\hline & 12 & & 56347627171 \\
\hline & 13 & & 624753010000 \\
\hline & 14 & & 6235474100000 \\
\hline & 15 & & 62354741000000 \\
\hline & 16 & & 623547410000000 \\
\hline & 17 & & 6235474100000000 \\
\hline \multirow[t]{7}{*}{ 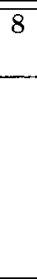 } & & $\mathbb{Z}_{4} \times \mathbb{Z} 2$ & $\begin{array}{l}\left\{x, y \mid x^{4}=y^{2}=i, x y=y x\right\} \\
0=i, 1=x, 2=x^{2}, 3=x^{3}, 4=y, 5=y x, 6=y x^{2}, 7=y x^{3}\end{array}$ \\
\hline & 12 & & 45361027100 \\
\hline & 13 & & 4331562710000 \\
\hline & 14 & & 7465237100000 \\
\hline & 15 & & 74652371000000 \\
\hline & 16 & & 746523710000000 \\
\hline & 17 & & 7465237100000000 \\
\hline \multirow[t]{7}{*}{ 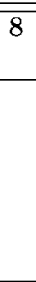 } & & $\mathbb{Z}_{2}^{3}$ & $\begin{array}{l}\left\{x, y, z \mid x^{2}=y^{2}=i, x y=y x, x z=z x, z y=y z,\right\} \\
0=i, 1=x, 2=y, 3=x y, 4=z, 5=x z, 6=y z, 7=x y z\end{array}$ \\
\hline & 8 & & 5643271 \\
\hline & 12 & & 46535271000 \\
\hline & 14 & & 4365120710000 \\
\hline & 15 & & 54364512710000 \\
\hline & 16 & & 643512710000000 \\
\hline & 17 & & 6435127100000000 \\
\hline \multirow[t]{7}{*}{8} & & $\overline{D_{4}}$ & $\begin{array}{l}\left\{x, y \mid x^{4}=y^{2}=i, x^{-1}=y x y\right\} \\
0=i, 1=x, 2=x^{2}, 3=x^{3}, 4=y, 5=x^{3} y, 6=x^{2} y, 7=x y\end{array}$ \\
\hline & 12 & & $2 \longdiv { 1 5 7 4 3 6 6 1 7 1 }$ \\
\hline & 13 & & 645527713100 \\
\hline & 14 & & 1243657100000 \\
\hline & 15 & & 12436571000000 \\
\hline & 16 & & 564243710000000 \\
\hline & 17 & & 5642437100000000 \\
\hline \multirow[t]{8}{*}{8} & & $\overline{Q_{8}}$ & $\begin{array}{l}\left\{x, y \mid x^{4}=y^{4}=i, y x y=x^{3}\right\} \\
0=i, 1=x, 2=x^{2}, 3=x^{3}, 4=y, 5=x^{3} y, 6=x^{2} y, 7=x y\end{array}$ \\
\hline & 11 & & 2630547100 \\
\hline & 12 & & 26334571000 \\
\hline & 13 & & 746523710000 \\
\hline & 14 & & 1145247066673 \\
\hline & 15 & & 45325610000000 \\
\hline & 16 & & 453256100000000 \\
\hline & 17 & & 4532561000000000 \\
\hline
\end{tabular}


Table A.2: Partial- $k$ No Fixed Point Cover Starters (continued)

\begin{tabular}{|c|c|c|c|}
\hline $\mathrm{v}$ & $\mathrm{k}$ & $G$ & \\
\hline 9 & & $\mathbb{Z}_{9}$ & \\
\hline & $\begin{array}{c}9 \\
13 \\
14 \\
15 \\
16\end{array}$ & 45628731 & $\begin{array}{llllllllllllll}5 & 3 & 4 & 1 & 2 & 2 & 6 & 8 & 7 & 1 & 0 & 0 & & \\
6 & 7 & 3 & 2 & 6 & 4 & 5 & 8 & 1 & 0 & 0 & 0 & 0 & \\
6 & 4 & 7 & 2 & 3 & 5 & 1 & 7 & 8 & 1 & 0 & 0 & 0 & 0 \\
6 & 4 & 8 & 4 & 3 & 2 & 5 & 7 & 8 & 1 & 0 & 0 & 0 & 0\end{array}$ \\
\hline 9 & & $Z_{3} \times Z_{3}$ & $\begin{array}{l}\left\{x, y \mid x^{3}=y^{3}=i, x y=y x\right\} \\
0=i, 1=x, 2=x^{2}, 3=y, 4=x y \\
5=x y^{2}, 6=, 7 x y=, 8=x^{2} y^{2}\end{array}$ \\
\hline & $\begin{array}{c}9 \\
13 \\
14 \\
15 \\
16\end{array}$ & & $\begin{array}{lllllllllllllll}4 & 5 & 6 & 2 & 8 & 7 & 3 & 1 & 0 & & & & & \\
6 & 2 & 4 & 7 & 5 & 1 & 3 & 7 & 8 & 1 & 0 & 0 & \\
2 & 3 & 0 & 2 & 7 & 6 & 4 & 5 & 8 & 8 & 1 & 0 & 0 \\
3 & 1 & 5 & 2 & 7 & 6 & 4 & 5 & 8 & 8 & 1 & 0 & 0 & 0 \\
6 & 2 & 3 & 4 & 0 & 2 & 7 & 5 & 8 & 1 & 0 & 0 & 0 & 0 & 0\end{array}$ \\
\hline 10 & & $\mathbb{Z}_{10}$ & \\
\hline & $\begin{array}{l}12 \\
13 \\
14 \\
15 \\
16 \\
17\end{array}$ & & $\begin{array}{lllllllllllllll}5 & 6 & 3 & 7 & 9 & 4 & 2 & 8 & 1 & 0 & 0 & & & & \\
8 & 4 & 6 & 5 & 6 & 9 & 3 & 1 & 7 & 2 & 2 & 1 & & & \\
3 & 5 & 9 & 8 & 2 & 8 & 6 & 7 & 4 & 9 & 1 & 0 & 0 & \\
8 & 4 & 5 & 7 & 1 & 6 & 3 & 7 & 9 & 2 & 9 & 1 & 0 & 0 \\
2 & 7 & 8 & 5 & 2 & 3 & 9 & 6 & 4 & 7 & 1 & 0 & 0 & 0 & 0 \\
5 & 3 & 9 & 6 & 7 & 7 & 6 & 2 & 4 & 8 & 1 & 0 & 0 & 0 & 0\end{array}$ \\
\hline 10 & & $\overline{D_{5}}$ & $\begin{array}{l}\left\{x, y \mid x^{5}=y^{2}=i, x^{-1}=y x y\right\} \\
0=i, 1=x, 2=x^{2}, 3=x^{3}, 4=x^{4} \\
5=y, 6=x^{4} y, 7=x^{3} y, 8=x^{2} y, 9=x y\end{array}$ \\
\hline & $\begin{array}{l}16 \\
17 \\
18 \\
19 \\
20\end{array}$ & & 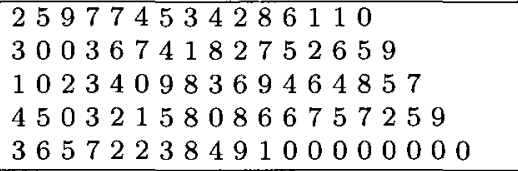 \\
\hline 11 & & $\mathbb{Z}_{11}$ & \\
\hline & $\begin{array}{l}15 \\
17 \\
18\end{array}$ & & $\begin{array}{l}104908751720346 \\
539567510104102368 \\
860341054316259790\end{array}$ \\
\hline
\end{tabular}


Table A.3: Entire- $k$ Single Fixed Point Cover Starters

\begin{tabular}{|c|c|c|c|}
\hline $\mathrm{v}$ & $\mathrm{k}$ & $G$ & \\
\hline 3 & & $\mathbb{Z}_{2}$ & \\
\hline & $\begin{array}{l}5 \\
6 \\
7 \\
8\end{array}$ & & 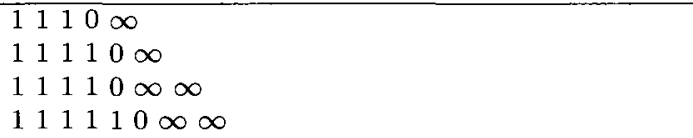 \\
\hline$\overline{4}$ & & $\overline{\mathbb{Z}_{3}}$ & \\
\hline & $\begin{array}{c}5 \\
6 \\
7 \\
8 \\
9 \\
10\end{array}$ & & 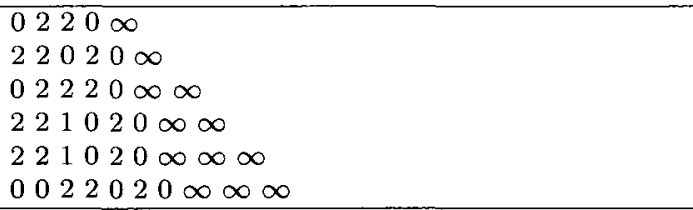 \\
\hline$\overline{5}$ & & $\mathbb{Z}_{4}$ & \\
\hline & $\begin{array}{c}7 \\
8 \\
9 \\
10 \\
\end{array}$ & & $\begin{array}{llllllll}1 & 3 & 3 & 2 & 3 & 1 & \infty \\
1 & 3 & 3 & 2 & 3 & 3 & 1 & \infty \\
0 & 1 & 0 & 1 & 3 & 3 & 0 & \infty \infty \\
3 & 2 & 2 & 0 & 2 & 3 & 3 & 0\end{array}$ \\
\hline 5 & & $\bar{K} K_{4}$ & $\left\{x, y \mid x^{2}=y^{2}=i, x y=y x\right\} 0=i, 1=x, 2=y, 3=x y$ \\
\hline & $\begin{array}{c}7 \\
9 \\
10 \\
11 \\
12 \\
\end{array}$ & & 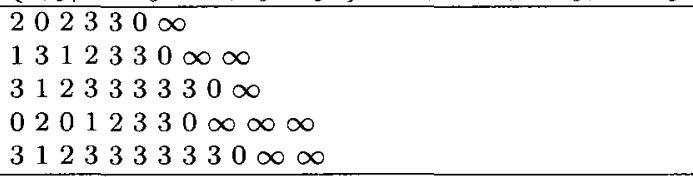 \\
\hline 6 & & $\mathbb{Z}_{5}$ & \\
\hline & $\begin{array}{c}9 \\
10 \\
11 \\
12\end{array}$ & & 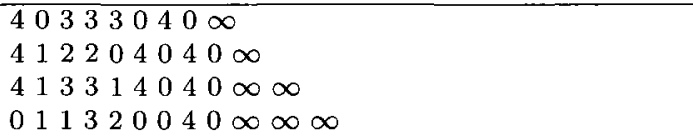 \\
\hline$\overline{7}$ & & $\mathbb{Z}_{6}$ & \\
\hline & $\begin{array}{l}10 \\
11 \\
12 \\
13 \\
14\end{array}$ & & 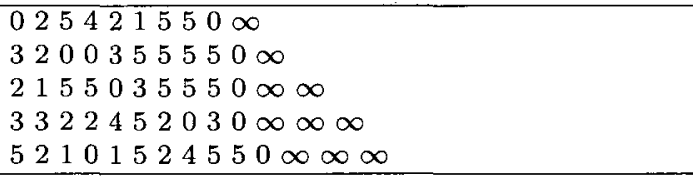 \\
\hline$\overline{77}$ & & 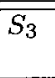 & $\begin{array}{l}\left\{x, y \mid x^{3}=y^{2}=i, x^{-1}=y x y\right\} \\
0=i, 1=x, 2=x^{2}, 3=y, 4=x^{2} y, 5=x y\end{array}$ \\
\hline & $\begin{array}{l}11 \\
12 \\
13 \\
14 \\
\end{array}$ & & 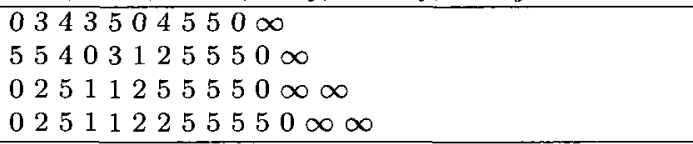 \\
\hline$\overline{8}$ & & $\overline{\mathbb{Z}_{7}}$ & \\
\hline & $\begin{array}{c}9 \\
11 \\
12 \\
13 \\
14\end{array}$ & & 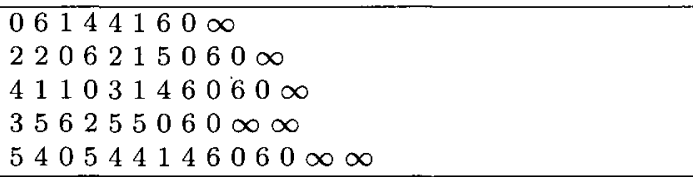 \\
\hline
\end{tabular}


Table A.3: Entire- $k$ Single Fixed Point Cover Starters (continued)

\begin{tabular}{|c|c|c|c|}
\hline $\mathrm{v}$ & $\mathrm{k}$ & $G$ & \\
\hline 9 & & $\mathbb{Z}_{8}$ & \\
\hline & $\begin{array}{l}13 \\
14 \\
15 \\
16 \\
17 \\
18\end{array}$ & & 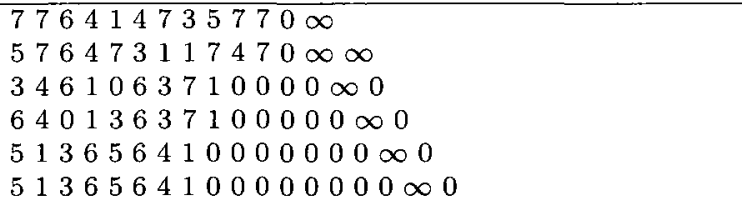 \\
\hline 9 & & $\mathbb{Z}_{4} \times \mathbb{Z} 2$ & $\begin{array}{l}\left\{x, y \mid x^{4}=y^{2}=i, x y=y x\right\} \\
0=i, 1=x, 2=x^{2}, 3=x^{3}, 4=y, 5=y x, 6=y x^{2}, 7=y x^{3}\end{array}$ \\
\hline & $\begin{array}{l}13 \\
14 \\
15 \\
16 \\
17 \\
18\end{array}$ & & 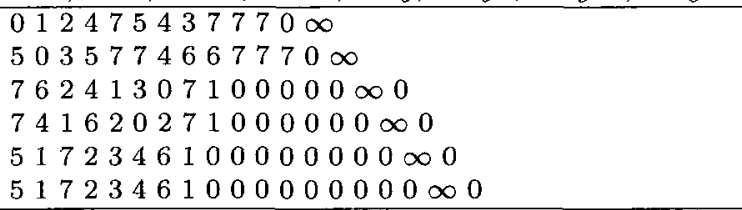 \\
\hline$\overline{99}$ & & $\overline{\mathbb{Z}_{2} \times \mathbb{Z} 2 \times \mathbb{Z} 2}$ & $\begin{array}{l}\left\{x, y, z \mid x^{2}=y^{2}=i, x y=y x, x z=z x, z y=y z,\right\} \\
0=i, 1=x, 2=y, 3=x y, 4=z, 5=x z, 6=y z, 7=x y z\end{array}$ \\
\hline & $\begin{array}{l}15 \\
17\end{array}$ & & 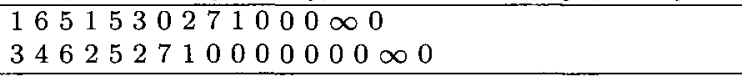 \\
\hline 9 & & $\overline{D_{4}}$ & $\begin{array}{l}\left\{x, y \mid x^{4}=y^{2}=i, x^{-1}=y x y\right\} \\
0=i, 1=x, 2=x^{2}, 3=x^{3}, 4=y, 5=x^{3} y, 6=x^{2} y, 7=x y\end{array}$ \\
\hline & $\begin{array}{l}13 \\
15 \\
16 \\
17 \\
18\end{array}$ & & 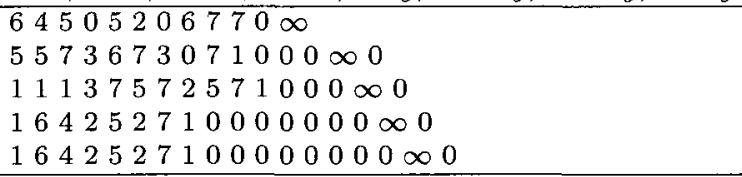 \\
\hline 9 & & $\overline{Q_{8}}$ & $\begin{array}{l}\left\{x, y \mid x^{4}=y^{4}=i, y x y=x^{3}\right\} \\
0=i, 1=x, 2=x^{2}, 3=x^{3}, 4=y, 5=x^{3} y, 6=x^{2} y, 7=x y\end{array}$ \\
\hline & $\begin{array}{l}13 \\
14 \\
15 \\
16 \\
17 \\
18\end{array}$ & & 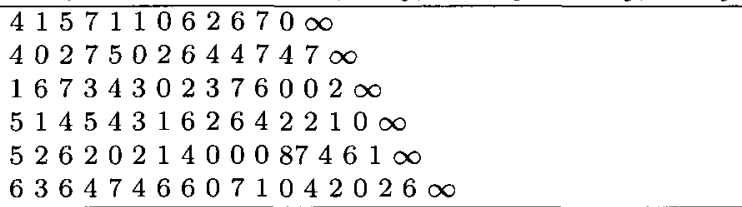 \\
\hline 10 & & $\mathbb{Z}_{9}$ & \\
\hline & $\begin{array}{l}15 \\
16 \\
17 \\
18 \\
19 \\
20 \\
21 \\
22\end{array}$ & & 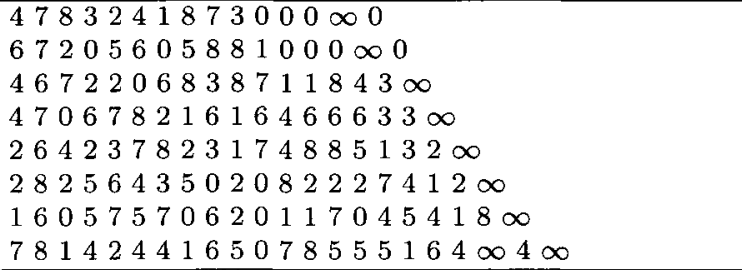 \\
\hline
\end{tabular}


Table A.3: Entire- $k$ Single Fixed Point Cover Starters (continued)

\begin{tabular}{|c|c|c|c|}
\hline $\mathrm{v}$ & $\mathrm{k}$ & $\bar{G}$ & \\
\hline$\overline{10}$ & & $\overline{\bar{Z} Z_{3} \times Z_{3}}$ & $\begin{array}{l}\left\{x, y \mid x^{3}=y^{3}=i, x y=y x\right\} \\
0=i, 1=x, 2=x^{2}, 3=y, 4=x y, 5=x y^{2}, 6=, 7 x y=, 8=x^{2} y^{2}\end{array}$ \\
\hline & $\begin{array}{l}15 \\
16 \\
17 \\
18 \\
19 \\
20\end{array}$ & & 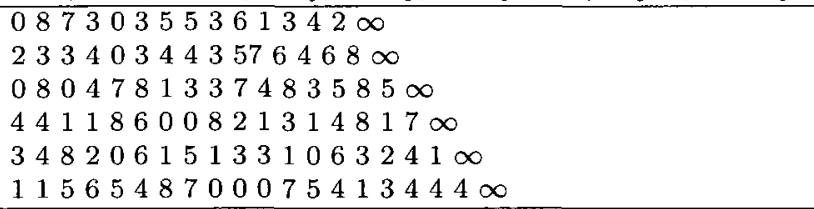 \\
\hline$\overline{111}$ & & $\overline{\mathbb{Z}_{10}}$ & \\
\hline & $\begin{array}{l}16 \\
17 \\
18 \\
19 \\
20 \\
21 \\
22\end{array}$ & & 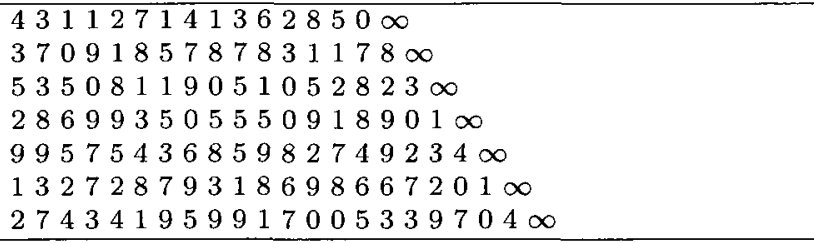 \\
\hline 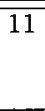 & & $\overline{D_{5}}$ & $\begin{array}{l}\left\{x, y \mid x^{5}=y^{2}=i, x^{-1}=y x y\right\} \\
0=i, 1=x, 2=x^{2}, 3=x^{3}, 4=x^{4}, 5=y \\
6=x^{4} y, 7=x^{3} y, 8=x^{2} y, 9=x y\end{array}$ \\
\hline & $\begin{array}{l}17 \\
18 \\
19 \\
20 \\
21 \\
22\end{array}$ & & 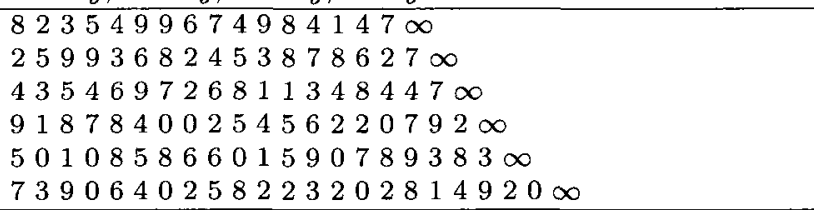 \\
\hline 12 & & $\mathbb{Z}_{11}$ & \\
\hline & $\begin{array}{l}17 \\
18 \\
19 \\
20 \\
21 \\
22 \\
23 \\
24 \\
\end{array}$ & & $\begin{array}{l}9626223414976157 \infty \\
101031695106206574 \infty \\
23927931041912554210 \infty \\
6423906843771931444 \infty \\
230085810438475986137 \infty \\
061005740710102033512193 \infty \\
101088065192744101020560 \infty \\
5233588669108538496110959 \infty\end{array}$ \\
\hline 13 & & $\mathbb{Z}_{12}$ & \\
\hline & $\begin{array}{l}19 \\
20 \\
21 \\
22 \\
23 \\
24 \\
25 \\
26 \\
27\end{array}$ & & $\begin{array}{l}411032057404101095933 \infty \\
20111058841160611110113810 \infty \\
928947256516098610004 \infty \\
69101001867376114511669611 \infty \\
949626057645102564288911 \infty \\
1028637237113576999231733 \infty \\
7135101168736041070010628088 \infty \\
11958604321021109214651108944 \infty \\
812671108767747103101195112357 \infty\end{array}$ \\
\hline
\end{tabular}


Table A.3: Entire- $k$ Single Fixed Point Cover Starters (continued)

\begin{tabular}{|c|c|c|c|}
\hline $\mathrm{v}$ & $\mathrm{k}$ & $G$ & \\
\hline 13 & & $Z_{6} \times Z_{2}$ & $\begin{array}{l}\{x, y \mid\} \\
0=i, 1=x, 2=x^{2}, 3=x^{3}, 4=x^{4}, 5=x^{5} \\
6=y, 7=x y, 8=x^{2} y, 9=x^{3} y, 10=x^{4} y, 11=x^{5} y\end{array}$ \\
\hline & $\begin{array}{l}19 \\
20 \\
21 \\
22 \\
23 \\
24 \\
25 \\
26 \\
27\end{array}$ & & $\begin{array}{l}231173133818211105914 \infty \\
1182268545761085657116 \infty \\
927945784380107314009 \infty \\
1175156189029384410876 \infty \\
564453871060881124315556 \infty \\
75213446151105011790864104 \infty \\
161033799104127205101009104113 \infty \\
34920686435104111126411081184 \infty \\
401011981110114814187255040633 \infty\end{array}$ \\
\hline 13 & & $\overline{D_{6}}$ & $\begin{array}{l}\left\{x, y \mid x^{6}=y^{2}=i, x^{-1}=y x y\right\} \\
0=i, 1=x, 2=x^{2}, 3=x^{3}, 4=x^{4}, 5=x^{5}, 6=y \\
7=x^{5} y, 8=x^{4} y, 9=x^{3} y, 10=x^{2} y, 11=x y\end{array}$ \\
\hline & $\begin{array}{l}19 \\
21 \\
22 \\
23 \\
24 \\
25 \\
26 \\
27\end{array}$ & & $\begin{array}{l}1191107937869003311107 \infty \\
667288151311802305847 \infty \\
41355870929511178388753 \infty \\
529119644505844510371922 \infty \\
247776781637603549128620 \infty \\
138467116161181023697011889 \infty \\
472011029141539834111180510112 \infty \\
1094036742010311100869560058511 \infty\end{array}$ \\
\hline 13 & & $\overline{A_{4}}$ & $\begin{array}{l}\left\{x, y \mid x^{3}=y^{2}=i, x y x=y x^{-1} y\right\} \\
0=i, 1=x, 2=x^{2}, 3=y, 4=x y, 5=x^{2} y \\
6=x^{2} y, 7=y x, 8=x y x, 9=x^{2} y x, 10=y x^{2}, 11=x^{2} y x^{2}\end{array}$ \\
\hline & $\begin{array}{l}19 \\
21 \\
22 \\
23 \\
24 \\
25 \\
26 \\
27\end{array}$ & & $\begin{array}{l}644535695069040339 \infty \\
116416069024599761011110 \infty \\
5242668101647910600881183 \infty \\
631171458061211158228626 \infty \\
612655910510697101132994741110 \infty \\
38110421691325865771111153 \infty \\
98100998094048771038841007211 \infty \\
110119782001079841140114639669 \infty\end{array}$ \\
\hline 13 & & $Z_{3} \rtimes Z_{4}$ & $\begin{array}{l}\{x, y \mid\} \\
0=\infty, 1=i, 2=x, 3=x^{2}, 4=x^{3}, 5=x^{4} \\
6=x^{5}, 7=y, 8=x^{5} y, 9=x^{4} y, 10=x^{3} y, 11=y x\end{array}$ \\
\hline & $\begin{array}{l}20 \\
21 \\
22 \\
23 \\
24 \\
25 \\
26 \\
27\end{array}$ & & $\begin{array}{l}810341021107105100034224 \infty \\
424951065111110460978396 \infty \\
244011530710311102724411739 \infty \\
714971161168928461177575 \infty \\
046511452163595662176360 \infty \\
311193112917571093682253896 \infty \\
90715591771100211134184212010 \infty \\
4358259434885601145428261089 \infty\end{array}$ \\
\hline
\end{tabular}


Table A.3: Entire- $k$ Single Fixed Point Cover Starters (continued)

\begin{tabular}{|c|c|c|c|}
\hline $\mathrm{v}$ & $\mathrm{k}$ & $G$ & \\
\hline 14 & & $\mathbb{Z}_{13}$ & \\
\hline & $\begin{array}{l}21 \\
22 \\
23 \\
24 \\
25 \\
26 \\
27 \\
28 \\
29\end{array}$ & & $\begin{array}{l}11812210212810481174971244 \infty \\
514128681212104111139111023 \infty \\
35181118756126375509851011 \infty \\
4592812412051003107551212151 \infty \\
1211101069107471279731066112311 \infty \\
4512212150821257675134599579 \infty \\
70107047987278712556099411314 \infty \\
18501051080691213056621211661031 \infty \\
115681180594876321312108882127570 \infty\end{array}$ \\
\hline 15 & & $\overline{\mathbb{Z}}_{14}$ & \\
\hline & $\begin{array}{l}24 \\
25 \\
26 \\
27 \\
28 \\
29 \\
30 \\
31\end{array}$ & & $\begin{array}{l}8772627731062460164111013571 \infty \\
61105911011184688171142101113122 \infty \\
99410631111112016113141371221312512 \infty \\
1131393470011133131263913472613610 \infty \\
3131211118741313525103631826121313131 \infty \\
36697479921813411121047313812386410 \infty \\
3241201111101121111180411271013045931211 \infty \\
1412963321213101108101191113011132821112103 \infty\end{array}$ \\
\hline 15 & & $D_{7}$ & $\begin{array}{l}\left\{x, y \mid x^{7}=y^{2}=i, x^{-1}=y x y\right\} \\
0=i, 1=x, 2=x^{2}, 3=x^{3}, 4=x^{4}, 5=x^{5}, 6=x^{6} \\
7=y, 8=x^{6} y, 9=x^{5} y, 10=x^{4} y, 11=x^{3} y, 12=x^{2} y, 13=x y\end{array}$ \\
\hline & $\begin{array}{l}25 \\
26 \\
27 \\
28 \\
29 \\
30 \\
31\end{array}$ & & $\begin{array}{l}470391124997513596351292212 \infty \\
61681133028339131112812129131271 \infty \\
13210553116811189569111010131062985 \infty \\
43211810110152177077341010274236 \infty \\
4912873113125133412111713842442210108 \infty \\
102791358624118294109788911121011157 \infty \\
68120710341243129104321306113445420110 \infty\end{array}$ \\
\hline 16 & & $\mathbb{Z}_{15}$ & \\
\hline & $\begin{array}{l}17 \\
25 \\
26 \\
27 \\
28 \\
29 \\
30 \\
31 \\
32 \\
33\end{array}$ & & $\begin{array}{l}120106131123321113610012 \infty \\
814831364131291414010121612131414413 \infty \\
814831364131291414010121612131414413 \infty \\
01440425110221291351124513101355911 \infty \\
957814714114437111166141212214137108112 \infty \\
77548114209131045396221225781319 \infty \infty \\
8910981010112191071750599609251240 \infty \\
27614112413411446715141167586051263011 \infty \\
1001422791460128832135118112372135309614 \infty \\
69397711414541355010051214457132813069 \infty\end{array}$ \\
\hline
\end{tabular}


Table A.4: Partial- $k$ Single Fixed Point Cover Starters

\begin{tabular}{|c|c|c|c|}
\hline $\mathrm{v}$ & $\mathrm{k}$ & $G$ & \\
\hline \multirow[t]{6}{*}{3} & & $\mathbb{Z}_{2}$ & \\
\hline & 4 & & $10 \infty$ \\
\hline & 5 & & $110 \infty$ \\
\hline & 6 & & $1110 \infty$ \\
\hline & 7 & & $1110 \infty \infty$ \\
\hline & 8 & & $1110 \infty \infty \infty$ \\
\hline \multirow[t]{5}{*}{4} & & $\mathbb{Z}_{3}$ & \\
\hline & 7 & & $12020 \infty$ \\
\hline & 8 & & $11020 \infty \infty$ \\
\hline & 9 & & $11020 \infty \infty \infty$ \\
\hline & 10 & & $112020 \infty \infty \infty$ \\
\hline \multirow[t]{6}{*}{5} & & $\mathbb{Z}_{4}$ & \\
\hline & 6 & & 21300 \\
\hline & 7 & & $22130 \infty$ \\
\hline & 8 & & $221330 \infty$ \\
\hline & 9 & & $221330 \infty \infty$ \\
\hline & 10 & & $211330 \infty \infty \infty$ \\
\hline \multirow[t]{5}{*}{$\overline{5}$} & & $K_{4}$ & $\left\{x, y \mid x^{2}=y^{2}=i, x y=y x\right\} \quad 0=i, 1=x, 2=y, 3=x y$ \\
\hline & 8 & & 1202300 \\
\hline & 9 & & $1110230 \infty$ \\
\hline & 10 & & $1312330 \infty \infty$ \\
\hline & 11 & & $12302330 \infty \infty$ \\
\hline \multirow[t]{5}{*}{ 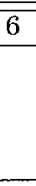 } & & $\mathbb{Z}_{5}$ & \\
\hline & 9 & & 24301400 \\
\hline & 10 & & $22131 \infty 40 \infty$ \\
\hline & 11 & & $33240140 \infty \infty$ \\
\hline & 12 & & $31224040 \infty \infty \infty$ \\
\hline \multirow[t]{7}{*}{7} & & $\mathbb{Z}_{6}$ & \\
\hline & 8 & & $312540 \infty$ \\
\hline & 10 & & $4021550 \infty$ \\
\hline & 11 & & $243125550 \infty$ \\
\hline & 12 & & $421413550 \infty \infty$ \\
\hline & 13 & & $54241355500 \infty$ \\
\hline & 14 & & $4514321550 \infty \infty \infty$ \\
\hline \multirow[t]{5}{*}{7} & & $\overline{S_{3}}$ & $\begin{array}{l}\left\{x, y \mid x^{3}=y^{2}=i, x^{-1}=y x y\right\} \\
0=i, 1=x, 2=x^{2}, 3=y, 4=x^{2} y, 5=x y\end{array}$ \\
\hline & 12 & & 23103455500 \\
\hline & 13 & & $02312455550 \infty$ \\
\hline & 14 & & $2124330450 \infty \infty \infty$ \\
\hline & 15 & & $5401230000000 \infty$ \\
\hline \multirow[t]{5}{*}{8} & & $\mathbb{Z}_{7}$ & \\
\hline & 12 & & $4624153060 \infty$ \\
\hline & 13 & & $23145026060 \infty$ \\
\hline & 14 & & $51243426060 \infty \infty$ \\
\hline & 15 & & 52034661000000 \\
\hline
\end{tabular}


Table A.4: Partial- $k$ Single Fixed Point Cover Starters (continued)

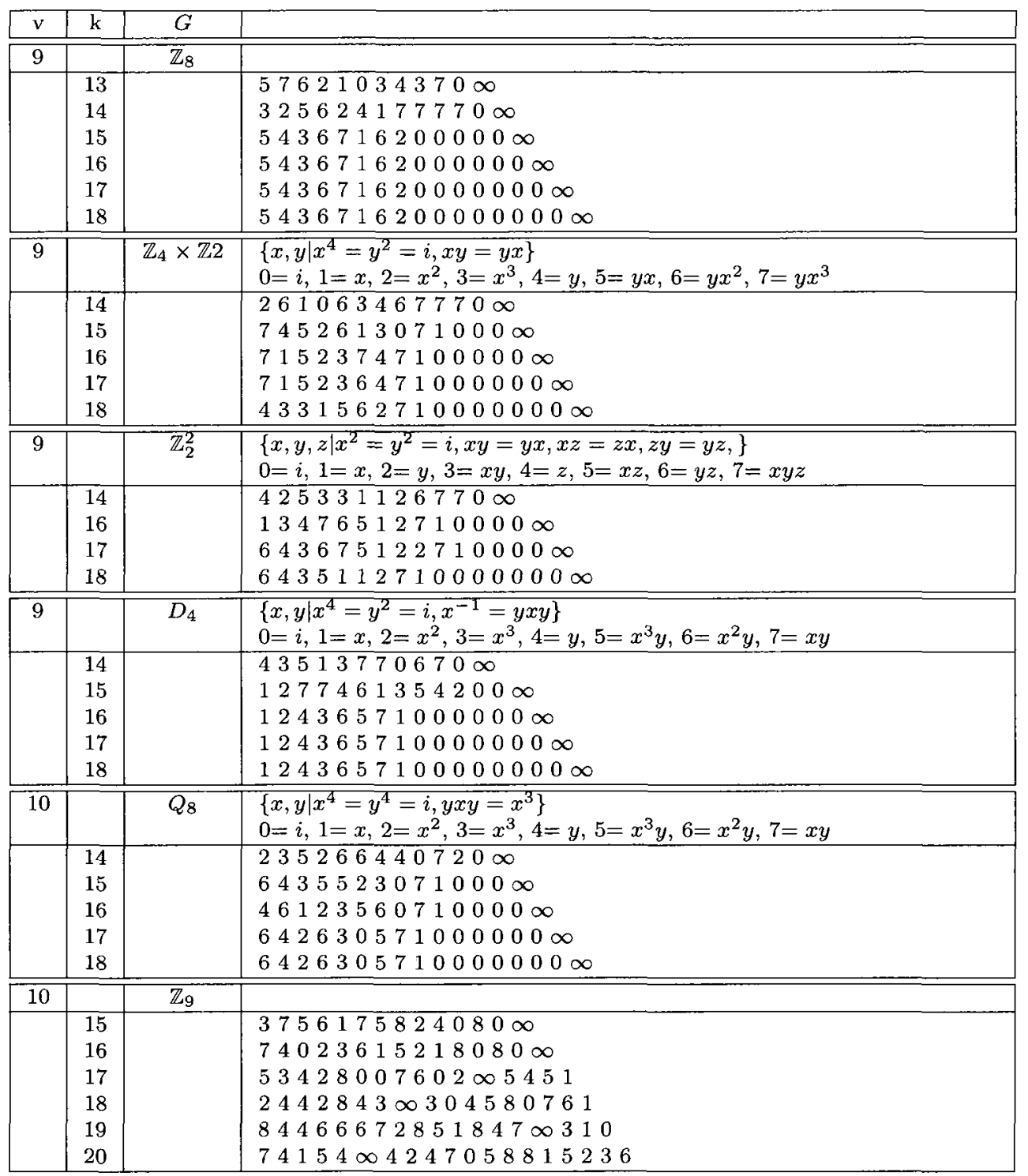


Table A.4: Partial- $k$ Single Fixed Point Cover Starters (continued)

\begin{tabular}{|c|c|c|c|}
\hline $\mathrm{v}$ & $\mathrm{k}$ & $G$ & \\
\hline 19 & & $Z_{3} \times Z_{3}$ & $\begin{array}{l}\left\{x, y \mid x^{3}=y^{3}=i, x y=y x\right\} \\
0=i, 1=x, 2=x^{2}, 3=y, 4=x y \\
5=x y^{2}, 6=, 7 x y=, 8=x^{2} y^{2}\end{array}$ \\
\hline & $\begin{array}{l}14 \\
15 \\
16 \\
17 \\
18 \\
19 \\
20\end{array}$ & & 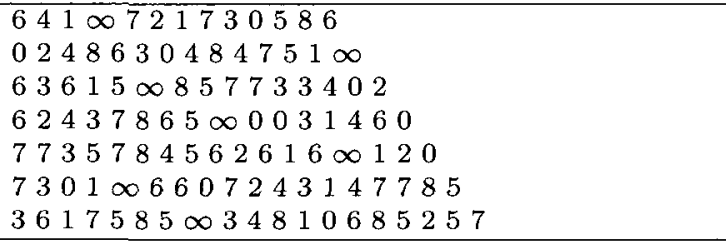 \\
\hline 11 & & $\mathbb{Z}_{10}$ & \\
\hline & $\begin{array}{l}12 \\
16 \\
17 \\
18 \\
19 \\
20 \\
21 \\
22\end{array}$ & & $\begin{array}{l}5637942810 \infty \\
0 \infty 5658427279301 \\
85277261348 \infty 03139 \\
8149321340 \infty 752168 \\
6574439215 \infty 51634708 \\
45570094217016112938 \\
3991004696 \infty 8528972730 \\
413199336298 \infty 012687054\end{array}$ \\
\hline 11 & & $\overline{D_{5}}$ & $\begin{array}{l}\left\{x, y \mid x^{5}=y^{2}=i, x^{-1}=y x y\right\} \\
0=i, 1=x, 2=x^{2}, 3=x^{3}, 4=x^{4}, 5=y \\
6=x^{4} y, 7=x^{3} y, 8=x^{2} y, 9=x y\end{array}$ \\
\hline & $\begin{array}{l}18 \\
19 \\
20 \\
21 \\
22 \\
23\end{array}$ & & 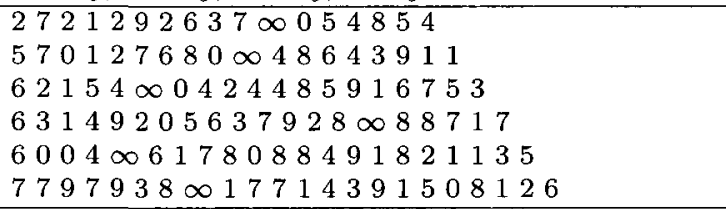 \\
\hline 12 & & $\mathbb{Z}_{11}$ & \\
\hline & $\begin{array}{l}18 \\
19 \\
20 \\
21 \\
22 \\
23 \\
24\end{array}$ & & $\begin{array}{l}0 \infty 88671039104180020 \\
\infty 64551744097158103247 \\
90881699978540102 \infty 3176 \\
98314498943621056 \infty 9570 \\
7655103511062993064518 \infty 710 \\
71778003192907862996 \infty 5410 \\
4066953749069023 \infty 89106187\end{array}$ \\
\hline 13 & & $\mathbb{Z}_{12}$ & \\
\hline & $\begin{array}{l}14 \\
18 \\
19 \\
20 \\
21 \\
22 \\
23 \\
24 \\
25 \\
26 \\
27\end{array}$ & & $\begin{array}{l}70 \infty 611089511324 \\
711973111110 \infty 3528406 \\
8431043109 \infty 6710591192 \\
541011112107810 \infty 3759468 \\
2 \infty 655832184564113010179 \\
111795 \infty 6467111151026108940 \\
11931010915473502720 \infty 10856 \\
24110102432810 \infty 411158407569 \\
66874480 \infty 117226410391711058 \\
3111700363719321074208910 \infty 5 \\
251053458990111211861073444 \infty 60\end{array}$ \\
\hline
\end{tabular}


Table A.4: Partial- $k$ Single Fixed Point Cover Starters (continued)

\begin{tabular}{|c|c|c|c|}
\hline $\mathrm{v}$ & $\mathrm{k}$ & $G$ & \\
\hline 13 & & $Z_{6} \times Z_{2}$ & $\begin{array}{l}\{x, y \mid\} \\
0=i, 1=x, 2=x^{2}, 3=x^{3}, 4=x^{4}, 5=x^{5} \\
6=y, 7=x y, 8=x^{2} y, 9=x^{3} y, 10=x^{4} y, 11=x^{5} y\end{array}$ \\
\hline & $\begin{array}{l}20 \\
21 \\
22 \\
23 \\
24 \\
25 \\
26 \\
27 \\
\end{array}$ & & $\begin{array}{l}711288779830 \infty 100175654 \\
8 \infty 4398111680610111121057 \\
2185511404887 \infty 2101111379623 \\
5151110164142975885 \infty 3102 \\
1019977537614011 \infty 01112810193 \\
2310241031 \infty 66211006748395211 \\
678161163112635108062 \infty 443189 \\
\infty 2005381134473111079104456001012\end{array}$ \\
\hline 13 & & $\overline{D_{6}}$ & $\begin{array}{l}\left\{x, y \mid x^{6}=y^{2}=i, x^{-1}=y x y\right\} \\
0=i, 1=x, 2=x^{2}, 3=x^{3}, 4=x^{4}, 5=x^{5} \\
6=y, 7=x^{5} y, 8=x^{4} y, 9=x^{3} y, 10=x^{2} y, 11=x y\end{array}$ \\
\hline & $\begin{array}{l}20 \\
22 \\
23 \\
24 \\
25 \\
26 \\
27\end{array}$ & & $\begin{array}{l}33410808299 \infty 61117101054 \\
48789 \infty 2911911167530751010 \\
9 \infty 110119410241169184476315 \\
1041011061149698311127 \infty 109135 \\
421501864979364107 \infty 3481165 \\
6474028013 \infty 41079476110841105 \\
4611 \infty 10031152504098418492967\end{array}$ \\
\hline 13 & & $\overline{A_{4}}$ & $\begin{array}{l}\left\{x, y \mid x^{3}=y^{2}=i, x y x=y x^{-1} y\right\} \\
0=i, 1=x, 2=x^{2}, 3=y, 4=x y, 5=x^{2} y \\
6=x^{2} y, 7=y x, 8=x y x, 9=x^{2} y x, 10=y x^{2}, 11=x^{2} y x^{2}\end{array}$ \\
\hline & $\begin{array}{l}20 \\
21 \\
22 \\
23 \\
24 \\
25 \\
26 \\
27\end{array}$ & & $\begin{array}{l}1833710 \infty 1142103111069795 \\
6000 \infty 279810115111985493 \\
13311119640 \infty 1012770510819 \\
956 \infty 13382118071086684515 \\
113 \infty 66610127128114110592045 \\
245384311011605101184679 \infty 472 \\
90610 \infty 392512753611101180411172 \\
79105054241625010363889011 \infty 91\end{array}$ \\
\hline 13 & & $Z_{3} \rtimes Z_{4}$ & $\begin{array}{l}\{x, y \mid\} \\
0=\infty, 1=i, 2=x, 3=x^{2}, 4=x^{3}, 5=x^{4} \\
6=x^{5}, 7=y, 8=x^{5} y, 9=x^{4} y, 10=x^{3} y, 11=y x\end{array}$ \\
\hline & $\begin{array}{l}21 \\
22 \\
23 \\
24 \\
25 \\
26 \\
27\end{array}$ & & $\begin{array}{l}340110 \infty 711571126911103810 \\
01071641089111329010 \infty 45110 \\
48411 \infty 03111566113071012489 \\
7155991201013101086881146 \infty 5 \\
1659710 \infty 32380682817104210511 \\
5425664 \infty 5026423231072117918 \\
75111101131297081115264098 \infty 083\end{array}$ \\
\hline
\end{tabular}


Table A.4: Partial- $k$ Single Fixed Point Cover Starters (continued)

\begin{tabular}{|c|c|c|c|}
\hline $\mathrm{v}$ & $\mathrm{k}$ & $G$ & \\
\hline \multirow[t]{11}{*}{14} & & $\mathbb{Z}_{13}$ & \\
\hline & 19 & & $690 \infty 10867163127246511$ \\
\hline & 21 & & $1210100792361117812505 \infty 4$ \\
\hline & 22 & & $73 \infty 512922436112509108271$ \\
\hline & 23 & & $\infty 66273157108930710512812114$ \\
\hline & 24 & & $9965248 \infty 1100771221031352128$ \\
\hline & 25 & & $3011902 \infty 4365801712712711141059$ \\
\hline & 26 & & $321011257256718490104241110 \infty 25$ \\
\hline & 27 & & $5104710931183410658 \infty 1221231272110$ \\
\hline & 28 & & $4112106872106 \infty 4109272331229457110$ \\
\hline & 29 & & $1174952101095122101216121013 \infty 10111211870$ \\
\hline \multirow[t]{9}{*}{15} & & $\mathbb{Z}_{14}$ & \\
\hline & 24 & & $9 \infty 30947138101561311682121103$ \\
\hline & 25 & & $81125 \infty 94590810528761111013364$ \\
\hline & 26 & & $7461321210181280134513125721311 \infty 93$ \\
\hline & 27 & & $712133172110466 \infty 117241851361209$ \\
\hline & 28 & & $1048080111131821321297822 \infty 5446101$ \\
\hline & 29 & & $0938961213212561087512010561 \infty 3211134$ \\
\hline & 30 & & $076208133202133124 \infty 2895703211910131$ \\
\hline & 31 & & $5442610370100541171213591081181012831 \infty$ \\
\hline \multirow[t]{9}{*}{15} & & $\overline{\overline{D_{7}}}$ & $\left\{x, y \mid x^{7}=y^{2}=i, x^{-1}=y x y\right\}$ \\
\hline & & & $0=i, 1=x, 2=x^{2}, 3=x^{3}, 4=x^{4}, 5=x^{5}, 6=x^{6}$ \\
\hline & & & $7=y, 8=x^{6} y, 9=x^{5} y, 10=x^{4} y, 11=x^{3} y, 12=x^{2} y, 13=x y$ \\
\hline & 26 & & $10168101071212113251181114349 \infty 150$ \\
\hline & 27 & & $8471136111093705 \infty 1319128913310211$ \\
\hline & 28 & & $804 \infty 319111223816512013710752111092$ \\
\hline & 29 & & $3601133521012 \infty 939128121111413539470$ \\
\hline & 30 & & $774812802 \infty 51012662910112864610410137$ \\
\hline & 31 & & $7111210831312001125710134443 \infty 139535101262$ \\
\hline \multirow[t]{8}{*}{16} & & $\mathbb{Z}_{15}$ & \\
\hline & 27 & & $125021351499877344661013411132 \infty 10$ \\
\hline & 28 & & $19 \infty 7301014413571311101120321481413679$ \\
\hline & 29 & & $129145412251118610 \infty 9387141266951307$ \\
\hline & 30 & & $14171213761410111217580 \infty 341441212614111329$ \\
\hline & 31 & & $11171014121463813111031414056106 \infty 4121384922$ \\
\hline & 32 & & 64127101315111014130071811389113141038241 \\
\hline & 33 & & $313091101191113613610647 \infty 988141610125297$ \\
\hline
\end{tabular}


Table A.5: Entire- $k$ Simple Double Fixed Point Cover Starters

\begin{tabular}{|c|c|c|c|}
\hline $\mathrm{v}$ & $\mathrm{k}$ & $\bar{G}$ & \\
\hline 4 & & $\overline{\mathbb{Z}_{2}}$ & \\
\hline & $\begin{array}{c}9 \\
10 \\
11 \\
12 \\
13\end{array}$ & & 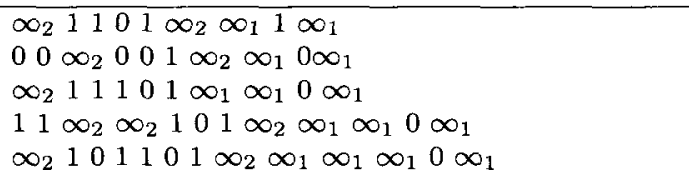 \\
\hline 5 & & $\mathbb{Z}_{3}$ & \\
\hline & $\begin{array}{l}10 \\
11 \\
12 \\
13 \\
14 \\
\end{array}$ & & 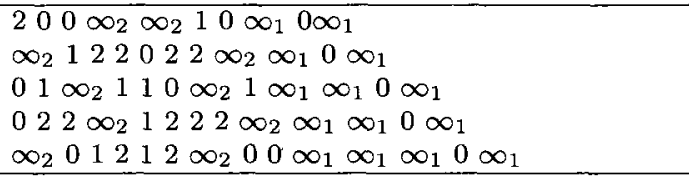 \\
\hline 6 & & $\overline{\mathbb{Z}_{4}}$ & \\
\hline & $\begin{array}{l}13 \\
14 \\
15 \\
16 \\
17\end{array}$ & & 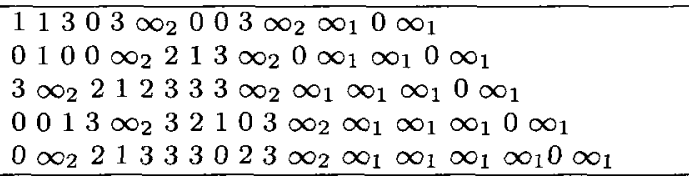 \\
\hline 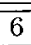 & & $\overline{K_{4}}$ & $\left\{x, y \mid x^{2}=y^{2}=i, x y=y x\right\} \quad 0=i, 1=x, 2=y, 3=x y$ \\
\hline & $\begin{array}{l}1 \overline{3} \\
14 \\
15 \\
16 \\
17\end{array}$ & & 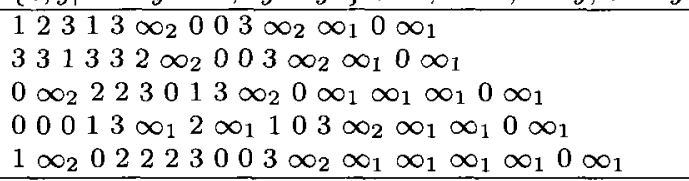 \\
\hline 7 & & $\overline{\mathbb{Z}_{5}}$ & \\
\hline & $\begin{array}{l}13 \\
14 \\
15 \\
16 \\
17 \\
18\end{array}$ & & 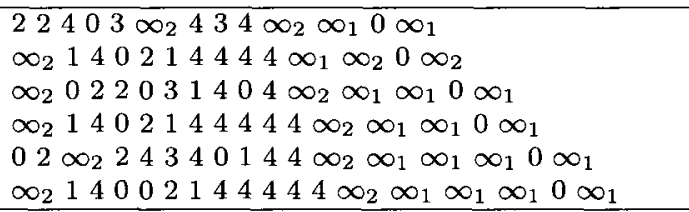 \\
\hline 8 & & $\overline{\mathbb{Z}_{6}}$ & \\
\hline & $\begin{array}{l}15 \\
16 \\
17 \\
18\end{array}$ & & 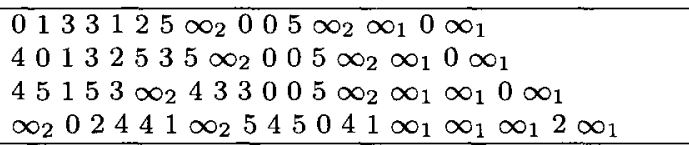 \\
\hline 8 & & $\overline{S_{3}}$ & $\begin{array}{l}\left\{x, y \mid x^{3}=y^{2}=i, x^{-1}=y x y\right\} \\
0=i, 1=x, 2=x^{2}, 3=y, 4=x^{2} y, 5=x y\end{array}$ \\
\hline & $\begin{array}{l}13 \\
15 \\
16 \\
17 \\
18 \\
\end{array}$ & & 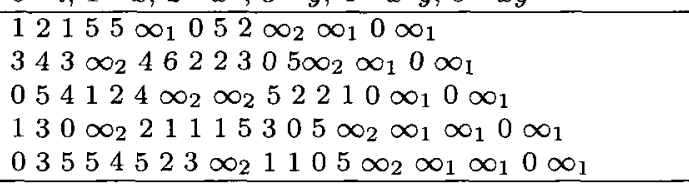 \\
\hline 8 & & $\overline{\mathbb{Z}_{7}}$ & \\
\hline & $\begin{array}{l}16 \\
17 \\
18\end{array}$ & & $\begin{array}{l}3 \infty_{2} 32 \infty_{1} 655516356 \infty_{2} 4 \infty_{1} \\
24 \infty_{2} 346636640 \infty_{2} 65 \infty_{1} \infty_{1} \\
11 \infty_{1} 323 \infty_{2} 20614 \infty_{2} 6043 \infty_{1}\end{array}$ \\
\hline
\end{tabular}


Table A.5: Entire- $k$ Simple Double Fixed Point Cover Starters (continued)

\begin{tabular}{|c|c|c|c|}
\hline $\mathrm{v}$ & $\mathrm{k}$ & $G$ & \\
\hline 9 & & $\mathbb{Z}_{8}$ & \\
\hline & $\begin{array}{l}18 \\
19 \\
20\end{array}$ & & $\begin{array}{l}\infty_{2} 5333 \infty_{2} 36344403507 \infty_{1} 2 \infty_{1} \\
31344377 \infty_{2} 47 \infty_{2} 4166262 \infty_{1} 5 \infty_{1} \\
4733635 \infty_{2} \infty_{2} 2075650 \infty_{1} 63 \infty^{2}\end{array}$ \\
\hline$\overline{99}$ & & $\overline{\mathbb{Z}_{4} \times \mathbb{Z} 2}$ & $\begin{array}{l}\left\{x, y \mid x^{4}=y^{2}=i, x y=y x\right\} \\
0=i, 1=x, 2=x^{2}, 3=x^{3}, 4=y, 5=y x, 6=y x^{2}, 7=y x^{3}\end{array}$ \\
\hline & $\begin{array}{l}18 \\
19 \\
20\end{array}$ & & $\begin{array}{l}\infty_{2} 3 \infty_{2} 574666131 \infty_{1} 37035 \infty_{1} \\
504 \infty_{2} 46615153234 \infty_{2} 1 \infty_{1} 54 \infty_{1} \\
7 \infty_{1} 22 \text { infty } 67050350 \infty_{2} 24042 \infty_{1}\end{array}$ \\
\hline 9 & & $D_{4}$ & $\begin{array}{l}\left\{x, y \mid x^{4}=y^{2}=i, x^{-1}=y x y\right\} \\
0=i, 1=x, 2=x^{2}, 3=x^{3}, 4=y, 5=x^{3} y, 6=x^{2} y, 7=x y\end{array}$ \\
\hline & $\begin{array}{l}19 \\
20\end{array}$ & & $\begin{array}{l}\infty_{2} 723 \infty_{2} 4004746 \infty_{1} 04257 \infty_{1} \\
40053 \infty_{1} 1441344134656 \infty_{2} 06 \infty_{2} \infty_{1}\end{array}$ \\
\hline 10 & & $\overline{Q_{8}}$ & $\begin{array}{l}\left\{x, y \mid x^{4}=y^{4}=i, y x y=x^{3}\right\} \\
0=i, 1=x, 2=x^{2}, 3=x^{3}, 4=y, 5=x^{3} y, 6=x^{2} y, 7=x y\end{array}$ \\
\hline & $\begin{array}{l}17 \\
18 \\
19 \\
20\end{array}$ & & 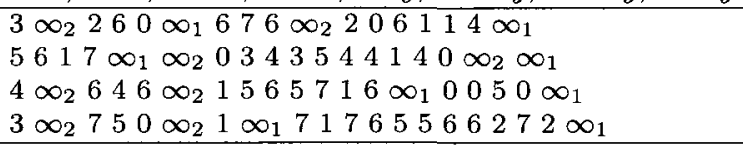 \\
\hline 10 & & $\mathbb{Z}_{9}$ & \\
\hline & $\begin{array}{l}19 \\
20 \\
21 \\
22\end{array}$ & & 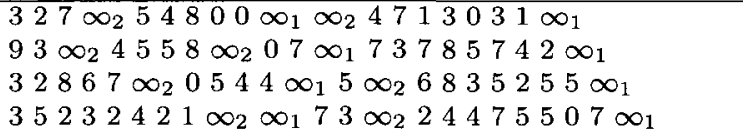 \\
\hline
\end{tabular}


Table A.6: Partial- $k$ Simple Double Fixed Point Cover Starters

\begin{tabular}{|c|c|c|c|}
\hline $\mathrm{v}$ & $\mathrm{k}$ & $G$ & \\
\hline 4 & & $\mathbb{Z}_{2}$ & \\
\hline & $\begin{array}{l}10 \\
11 \\
12 \\
13\end{array}$ & & 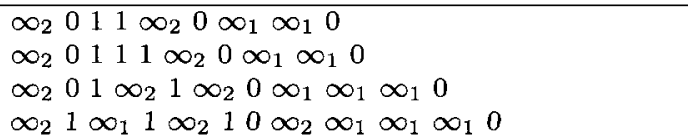 \\
\hline$\overline{5}$ & & $\overline{\mathbb{Z}_{3}}$ & \\
\hline & $\begin{array}{l}10 \\
11 \\
12 \\
13 \\
14\end{array}$ & & 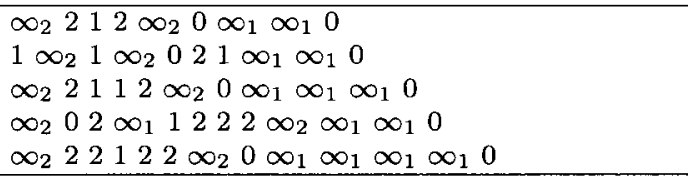 \\
\hline 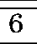 & & $\overline{\mathbb{Z}_{4}}$ & \\
\hline & $\begin{array}{l}12 \\
13 \\
14 \\
15 \\
16\end{array}$ & & $\begin{array}{lllllllllllll}\infty_{2} & 1 & 2 & 2 & 1 & \infty_{2} & 3 & 1 & \infty_{1} \infty_{1} & 0 \\
1 & 0 & 0 & \infty_{2} & 2 & 1 & 3 & \infty_{2} & 0 & \infty_{1} & \infty_{1} & 0 \\
\infty_{2} & 2 & 1 & 1 & 2 & 1 & 3 & \infty_{2} & 0 & \infty_{1} \infty_{1} & \infty_{1} 0 \\
1 \infty_{2} & 0 & 2 & 1 & 3 & 3 & 3 & \infty_{1} & \infty_{2} & \infty_{1} \infty_{1} \infty_{1} & 0 \\
\infty_{2} & 2 & 1 & 1 & 1 & 2 & 0 & 3 & \infty_{2} & 0 & \infty_{1} & \infty_{1} \infty_{1} \infty_{1} & 0\end{array}$ \\
\hline 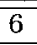 & & $\overline{K_{4}}$ & $\left\{x, y \mid x^{2}=y^{2}=i, x y=y x\right\} \quad 0=i, 1=x, 2=y, 3=x y$ \\
\hline & $\begin{array}{l}12 \\
13 \\
14 \\
15 \\
\end{array}$ & & $\begin{array}{lllllllllllllllll} & 0 & 1 & \infty_{2} & 2 & \infty_{2} & 3 & 1 & \infty_{1} & \infty_{1} & 0 \\
1 & 2 & 3 & \infty_{2} & 2 & 1 & 3 & \infty_{2} & 0 & \infty_{1} & \infty_{1} & 0 \\
1 & \infty_{2} & 2 & 3 & 2 & 1 & 3 & \infty_{2} & 0 & \infty_{1} & \infty_{1} & \infty_{1} & 0 \\
0 & 2 & 1 & \infty_{1} & 1 & \infty_{2} & 2 & 3 & 3 & \infty_{2} & \infty_{1} & \infty_{1} & \infty_{1} & 0 \\
\end{array}$ \\
\hline 7 & & $\mathbb{Z}_{5}$ & \\
\hline & $\begin{array}{l}13 \\
14 \\
15 \\
16\end{array}$ & & $\begin{array}{l}44412 \infty_{2} 3 \infty_{2} 31 \infty_{1} \infty_{1} 0 \\
2 \infty_{2} 3122144 \infty_{2} 0 \infty_{1} \infty_{1} 0 \\
31 \infty_{2} 12 \infty_{2} 0441 \infty_{1} \infty_{1} \infty_{1} 0 \\
10 \infty_{2} 0333244 \infty_{2} 0 \infty_{1} \infty_{1} \infty_{1} 0\end{array}$ \\
\hline 8 & & $\mathbb{Z}_{6}$ & \\
\hline & $\begin{array}{l}14 \\
15 \\
16 \\
17 \\
18\end{array}$ & & 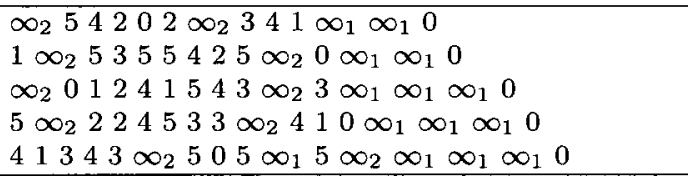 \\
\hline 8 & & $\overline{S_{3}}$ & $\begin{array}{l}\left\{x, y \mid x^{3}=y^{2}=i, x^{-1}=y x y\right\} \\
0=i, 1=x, 2=x^{2}, 3=y, 4=x^{2} y, 5=x y\end{array}$ \\
\hline & $\begin{array}{l}16 \\
17 \\
18 \\
19\end{array}$ & & $\begin{array}{l}\infty_{2} 444544133215 \infty_{2} 0 \infty_{1} \infty_{1} 0 \\
02 \infty_{2} 5554133415 \infty_{2} 0 \infty_{1} \infty_{1} 0 \\
1325510 \infty_{1} 3 \infty_{2} 3355 \infty_{2} \infty_{1} \infty_{1} \infty_{1} 0\end{array}$ \\
\hline
\end{tabular}


Table A.7: Entire- $k$ Augmented Double Fixed Point Cover Starters

\begin{tabular}{|c|c|c|c|}
\hline $\mathrm{v}$ & $\mathrm{k}$ & $G$ & \\
\hline \multirow[t]{6}{*}{4} & & $\mathbb{Z}_{2}$ & \\
\hline & 6 & & $10 \infty_{2} 11 \infty_{1}$ \\
\hline & 7 & & $\infty_{2} 100000 \infty_{1}$ \\
\hline & 8 & & $\infty_{2} 1000000 \infty_{1}$ \\
\hline & 9 & & $\infty_{2} 1100000000 \infty_{1}$ \\
\hline & 10 & & $\infty_{2} 10000000000 \infty_{1}$ \\
\hline \multirow[t]{6}{*}{5} & & $\mathbb{Z}_{3}$ & \\
\hline & 7 & & $\infty_{2} 01110 \infty_{1}$ \\
\hline & 8 & & $\infty_{2} 012100 \infty_{1}$ \\
\hline & 9 & & $\infty_{2} 0121000 \infty_{1}$ \\
\hline & 10 & & $\infty_{2} 01210000 \infty_{1}$ \\
\hline & 11 & & $\infty_{2} 1210000000 \infty_{1}$ \\
\hline \multirow[t]{6}{*}{6} & & $\mathbb{Z}_{4}$ & \\
\hline & 9 & & $\infty_{2} 03323 \quad 100 \infty_{1}$ \\
\hline & 10 & & $\infty_{2} 23031000 \infty_{1}$ \\
\hline & 11 & & $\infty_{2} 031200000 \infty_{1}$ \\
\hline & 12 & & $\infty_{2} 0312000000 \infty_{1}$ \\
\hline & 13 & & $\infty_{2} 12310000000 \infty_{1}$ \\
\hline \multirow[t]{6}{*}{$\overline{6}$} & & $K_{4}$ & $\left\{x, y \mid x^{2}=y^{2}=i, x y=y x\right\} 0=i, 1=x, 2=y, 3=x y$ \\
\hline & 9 & & $\infty_{2} 1213310 \infty_{1}$ \\
\hline & 11 & & $\infty_{2} 210310000 \infty_{1}$ \\
\hline & 12 & & $\infty_{2} 2103100000 \infty_{1}$ \\
\hline & 13 & & $\infty_{2} 12310000000 \infty_{1}$ \\
\hline & 14 & & $\infty_{2} 12331000000000 \infty_{1}$ \\
\hline \multirow[t]{5}{*}{$\overline{7} 7$} & & $\mathbb{Z}_{5}$ & \\
\hline & 11 & & $\infty_{2} 102310000 \infty_{1}$ \\
\hline & 12 & & $\infty_{2} 10223100000 \infty_{1}$ \\
\hline & 13 & & $\infty_{2} 102310000000 \infty_{1}$ \\
\hline & 14 & & $\infty_{2} 100231000000000 \infty_{1}$ \\
\hline \multirow[t]{5}{*}{8} & & $\overline{\mathbb{Z}_{6}}$ & \\
\hline & 12 & & $\infty_{2} 1340052000 \infty_{1}$ \\
\hline & 13 & & $\infty_{2} 23051200000 \infty_{1}$ \\
\hline & 14 & & $\infty_{2} 230512000000 \infty_{1}$ \\
\hline & 15 & & $\infty_{2} 2305120000000 \infty_{1}$ \\
\hline \multirow[t]{5}{*}{$\overline{8}$} & & $\overline{S_{3}}$ & $\begin{array}{l}\left\{x, y \mid x^{3}=y^{2}=i, x^{-1}=y x y\right\} \\
0=i, 1=x, 2=x^{2}, 3=y, 4=x^{2} y, 5=x y\end{array}$ \\
\hline & 13 & & $\infty_{2} 1514151000 \infty_{1}$ \\
\hline & 14 & & $\infty_{2} 40351230000 \infty_{1}$ \\
\hline & 15 & & $\infty_{2} 103145300000 \infty_{1}$ \\
\hline & 16 & & $\infty_{2} 1031453000000 \infty_{1}$ \\
\hline \multirow[t]{5}{*}{$\overline{99}$} & & $\overline{\mathbb{Z}_{7}}$ & \\
\hline & 13 & & $\infty_{2} 01663336610 \infty_{1}$ \\
\hline & 14 & & $\infty_{2} 011521361000 \infty_{1}$ \\
\hline & 15 & & $\infty_{2} 3145052100000 \infty_{1}$ \\
\hline & 16 & & $\infty_{2} 51204361000000 \infty_{1}$ \\
\hline
\end{tabular}


Table A.7: Entire- $k$ Augmented Double Point Cover Starters (continued)

\begin{tabular}{|c|c|c|c|}
\hline $\mathrm{v}$ & $\mathrm{k}$ & $G$ & \\
\hline 10 & & $\mathbb{Z}_{8}$ & \\
\hline & $\begin{array}{l}14 \\
15 \\
16 \\
17 \\
18\end{array}$ & & 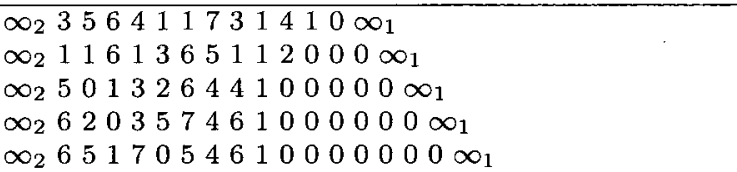 \\
\hline 10 & & $\overline{\mathbb{Z}_{4} \times \mathbb{Z} 2}$ & $\begin{array}{l}\left\{x, y \mid x^{4}=y^{2}=i, x y=y x\right\} \\
0=i, 1=x, 2=x^{2}, 3=x^{3}, 4=y, 5=y x, 6=y x^{2}, 7=y x^{3}\end{array}$ \\
\hline & $\begin{array}{l}15 \\
16 \\
17 \\
18\end{array}$ & & $\begin{array}{l}\infty_{2} 74222664707100 \infty_{1} \\
\infty_{2} 741126420071000 \infty_{1} \\
\infty_{2} 7525344061000000 \infty_{1} \\
\infty_{2} 70274436271000000 \infty_{1}\end{array}$ \\
\hline$\overline{10}$ & & $\overline{\mathbb{Z}_{2}^{3}}$ & $\begin{array}{l}\left\{x, y, z \mid x^{2}=y^{2}=i, x y=y x, x z=z x, z y=y z,\right\} \\
0=i, 1=x, 2=y, 3=x y, 4=z, 5=x z, 6=y z, 7=x y z\end{array}$ \\
\hline 10 & & $\overline{D_{4}}$ & $\begin{array}{l}\left\{x, y \mid x^{4}=y^{2}=i, x^{-1}=y x y\right\} \\
0=i, 1=x, 2=x^{2}, 3=x^{3}, 4=y, 5=x^{3} y, 6=x^{2} y, 7=x y\end{array}$ \\
\hline & $\begin{array}{l}15 \\
17 \\
18 \\
\end{array}$ & & 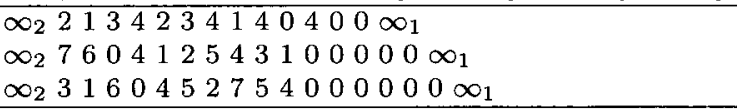 \\
\hline 10 & & $\overline{Q_{8}}$ & $\begin{array}{l}\left\{x, y \mid x^{4}=y^{4}=i, y x y=x^{3}\right\} \\
0=i, 1=x, 2=x^{2}, 3=x^{3}, 4=y, 5=x^{3} y, 6=x^{2} y, 7=x y\end{array}$ \\
\hline & $\begin{array}{l}15 \\
16 \\
17 \\
18\end{array}$ & & 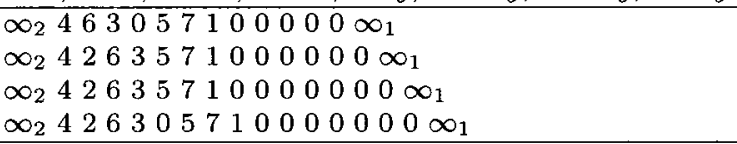 \\
\hline
\end{tabular}


Table A.8: Partial- $k$ Augmented Double Fixed Point Cover Starters

\begin{tabular}{|c|c|c|c|}
\hline $\mathrm{v}$ & $\mathrm{k}$ & $G$ & \\
\hline 4 & & $\mathbb{Z}_{2}$ & \\
\hline & $\begin{array}{l}6 \\
7 \\
8 \\
9\end{array}$ & & 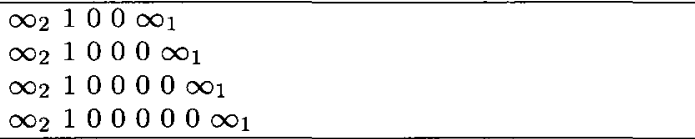 \\
\hline 5 & & $\mathbb{Z}_{3}$ & \\
\hline & $\begin{array}{c}7 \\
8 \\
9 \\
10 \\
\end{array}$ & & $\begin{array}{llllllll}2 & \infty_{2} & 0 & 1 & 0 & \infty_{1} \\
\infty_{2} & 1 & 2 & 1 & 0 & 0 & \infty_{1} \\
\infty_{2} & 1 & 2 & 1 & 0 & 0 & 0 & \infty_{1} \\
\infty_{2} & 1 & 2 & 1 & 0 & 0 & 0 & 0 \infty_{1}\end{array}$ \\
\hline 6 & & $\mathbb{Z}_{4}$ & \\
\hline & $\begin{array}{c}9 \\
10 \\
11 \\
12 \\
\end{array}$ & & 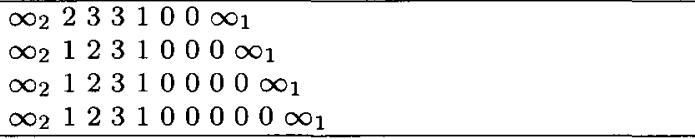 \\
\hline 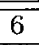 & & $\overline{K_{4}}$ & $\left\{x, y \mid x^{2}=y^{2}=i, x y=y x\right\} \quad 0=i, 1=x, 2=y, 3=x y$ \\
\hline & $\begin{array}{l}10 \\
11 \\
12 \\
13 \\
\end{array}$ & & 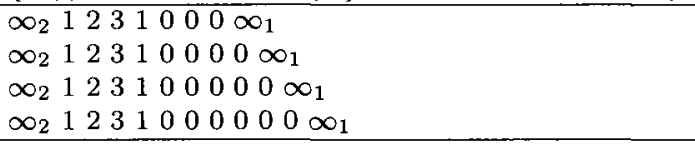 \\
\hline 7 & & $\mathbb{Z}_{5}$ & \\
\hline & $\begin{array}{c}9 \\
10 \\
11 \\
12 \\
13 \\
\end{array}$ & & 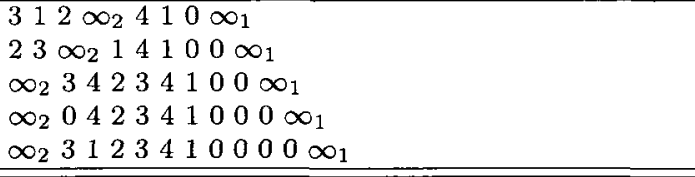 \\
\hline 8 & & $\mathbb{Z}_{6}$ & \\
\hline & $\begin{array}{l}11 \\
12 \\
13 \\
14 \\
\end{array}$ & & 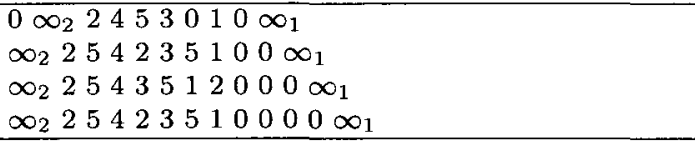 \\
\hline 8 & & $S_{3}$ & $\begin{array}{l}\left\{x, y \mid x^{3}=y^{2}=i, x^{-1}=y x y\right\} \\
0=i, 1=x, 2=x^{2}, 3=y, 4=x^{2} y, 5=x y\end{array}$ \\
\hline & $\begin{array}{l}13 \\
14 \\
15 \\
16 \\
\end{array}$ & & 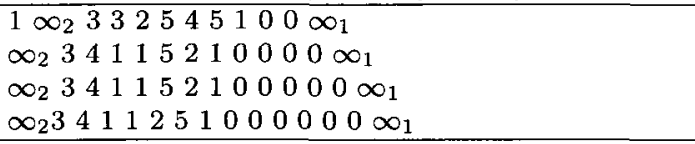 \\
\hline 9 & & $\mathbb{Z}_{7}$ & \\
\hline & $\begin{array}{l}13 \\
14 \\
15 \\
16 \\
\end{array}$ & & 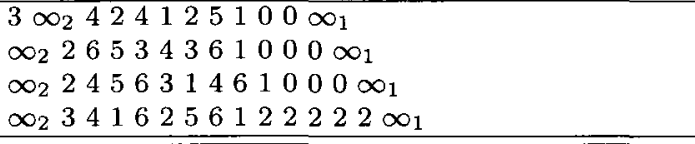 \\
\hline 10 & & $\overline{\mathbb{Z}_{8}}$ & \\
\hline & $\begin{array}{l}15 \\
16 \\
17 \\
18\end{array}$ & & 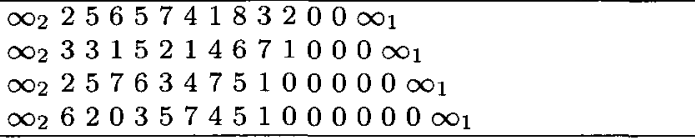 \\
\hline
\end{tabular}


Table A.8: Partial- $k$ Augmented Double Fixed Point Cover Starters (continued)

\begin{tabular}{|c|c|c|c|}
\hline$v$ & $\mathrm{k}$ & $G$ & \\
\hline 10 & & $\overline{\mathbb{Z}_{4} \times \mathbb{Z} 2}$ & $\begin{array}{l}\left\{x, y \mid x^{4}=y^{2}=i, x y=y x\right\} \\
0=i, 1=x, 2=x^{2}, 3=x^{3}, 4=y, 5=y x, 6=y x^{2}, 7=y x^{3}\end{array}$ \\
\hline & $\begin{array}{l}15 \\
16 \\
17 \\
18\end{array}$ & & 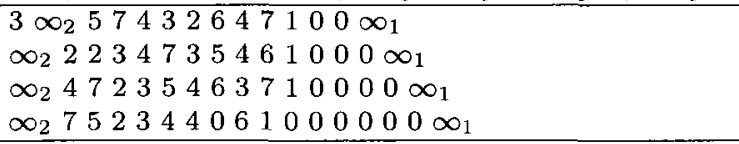 \\
\hline$\overline{10}$ & & $\overline{\mathbb{Z}_{2}^{3}}$ & $\begin{array}{l}\left\{x, y, z \mid x^{2}=y^{2}=i, x y=y x, x z=z x, z y=y z,\right\} \\
0=i, 1=x, 2=y, 3=x y, 4=z, 5=x z, 6=y z, 7=x y z\end{array}$ \\
\hline & & $\overline{D_{4}}$ & $\begin{array}{l}\left\{x, y \mid x^{4}=y^{2}=i, x^{-1}=y x y\right\} \\
0=i, 1=x, 2=x^{2}, 3=x^{3}, 4=y, 5=x^{3} y, 6=x^{2} y, 7=x y\end{array}$ \\
\hline & $\begin{array}{l}16 \\
17\end{array}$ & & $\begin{array}{l}\infty_{2} 16455372142000 \infty_{1} \\
\infty_{2} 166673514542000 \infty_{1}\end{array}$ \\
\hline & & $Q_{8}$ & $\begin{array}{l}\left\{x, y \mid x^{4}=y^{4}=i, y x y=x^{3}\right\} \\
0=i, 1=x, 2=x^{2}, 3=x^{3}, 4=y, 5=x^{3} y, 6=x^{2} y, 7=x y\end{array}$ \\
\hline & $\begin{array}{l}15 \\
16 \\
17 \\
18\end{array}$ & & 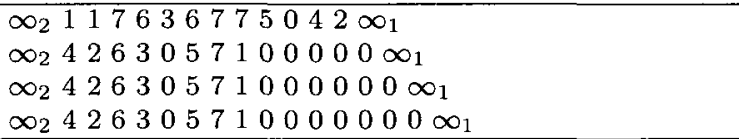 \\
\hline
\end{tabular}




\section{Bibliography}

[1] T. Beth, D. Jungnickel, and H. Lenz. Design theory. Vol. I, volume 69 of Encyclopedia of Mathematics and its Applications. Cambridge University Press, Cambridge, second edition, 1999.

[2] T. Beth, D. Jungnickel, and H. Lenz. Design theory. Vol. II, volume 78 of Encyclopedia of Mathematics and its Applications. Cambridge University Press, Cambridge, second edition, 1999.

[3] N. L. Biggs. Discrete mathematics. Oxford Science Publications. The Clarendon Press Oxford University Press, New York, second edition, 1989.

[4] R. C. Bryce and C. J. Colbourn. Prioritized interaction testing for pairwise coverage with seeding and contraints. Information and Software Technology Journal (IST), Elsevier, 48:2006, 2006.

[5] K. Burr and W. Young. Combinatorial test techniques: Table-based automation, test generation and code coverage. In Proceedings of the Intl. Conf. on Software Testing Analysis and Review, pages 503-513. West, 1998. 
[6] M. A. Chateauneuf, C. J. Colbourn, and D. L.Kreher. Covering arrays of strength three. Des. Codes Cryptogr., 16(3):235-242, 1999.

[7] D. Cohen, S. Dalal, M. Fredman, and G. Patton. The aetg system: an approach to testing based on combinatorial design. Software Engineering, IEEE Transactions on, 23(7):437-444, Jul 1997.

[8] C. J. Colbourn. Combinatorial aspects of covering arrays. Matematiche (Catania), 59(1-2):125-172 (2006), 2004.

[9] C. J. Colbourn and J. H. Dinitz, editors. Handbook of combinatorial designs. Discrete Mathematics and its Applications (Boca Raton). Chapman \& Hall/CRC, Boca Raton, FL, second edition, 2007.

[10] C. J. Colbourn, S. S. Martirosyan, G. L. Mullen, D. Shasha, G. B. Sherwood, and J. L. Yucas. Products of mixed covering arrays of strength two. J. Combin. Des., 14(2):124-138, 2006.

[11] J. D. Dixon and B. Mortimer. Permutation groups, volume 163 of Graduate Texts in Mathematics. Springer-Verlag, New York, 1996.

[12] D. S. Dummit and R. M. Foote. Abstract algebra. John Wiley \& Sons Inc., Hoboken, NJ, third edition, 2004.

[13] A. Hartman. Software and hardware testing using combinatorial covering suites. In Graph theory, combinatorics and algorithms, pages 237266. Springer, New York, 2005. 
[14] G. O. H. Katona. Two applications (for search theory and truth functions) of Sperner type theorems. Period. Math. Hungar., 3:19-26, 1973. Collection of articles dedicated to the memory of Alfréd Rényi, II.

[15] D. E. Knuth. The art of computer programming. Vol. 4, Fasc. 2. Addison-Wesley, Upper Saddle River, NJ, 2005. Generating all tuples and permutations.

[16] D. L. Kreher and D. R. Stinson. Combinatorial Algorithms. CRC Press, Boca Raton, FL, 1999. Generation, enumeration, and search.

[17] D. Kuhn, D. Wallace, and J. Gallo, A.M. Software fault interactions and implications for software testing. Software Engineering, IEEE Transactions on, 30(6):418-421, June 2004.

[18] H. B. Mann. On the construction of sets of orthogonal Latin squares. Ann. Math. Statistics, 14:401-414, 1943.

[19] K. Meagher. Covering Arrays on Graphs: Qualitative Independence Graphs and Extremal Set Partition Theory. PhD thesis, University of Ottawa, Ottawa, 2005.

[20] K. Meagher. Group construction of covering arrays - part 2. Technical report, University of Ottawa, 2005.

[21] K. Meagher and B. Stevens. Group construction of covering arrays. $J$. Combin. Des., 13(1):70-77, 2005. 
[22] A. H. Ronneseth and C. J. Colbourn. Merging covering arrays and compressing multiple sequence alignments. Discrete Appl. Math., 157(9):2177-2190, 2009.

[23] G. B. Sherwood, S. S. Martirosyan, and C. J. Colbourn. Covering arrays of higher strength from permutation vectors. J. Combin. Des., 14(3):202-213, 2006.

[24] B. Stevens. Transversal Covers and Packings. PhD thesis, University of Toronto, Toronto, 1998.

[25] D. R. Stinson. Combinatorial designs. Springer-Verlag, New York, 2004. Constructions and analysis, With a foreword by Charles J. Colbourn.

[26] H. Taylor and J. H. Dinitz. Costas Arrays, chapter VI.9, pages 357-361. In Colbourn and Dinitz [9], second edition, 2007.

[27] R. A. Walker, II and C. J. Colbourn. Tabu search for covering arrays using permutation vectors. J. Statist. Plann. Inference, 139(1):69-80, 2009.

[28] H. Wielandt. Finite permutation groups. Translated from the German by R. Bercov. Academic Press, New York, 1964.

[29] C. Yilmaz, M. Cohen, and A. Porter. Covering arrays for efficient fault characterization in complex configuration spaces. Software Engineering, IEEE Transactions on, 32(1):20-34, Jan. 2006.

[30] J. Yin. Constructions of difference covering arrays. J. Combin. Theory Ser. A, 104(2):327-339, 2003. 
[31] J. Yin. Cyclic difference packing and covering arrays. Des. Codes Cryptogr., 37(2):281-292, 2005. 